Supporting Information

\title{
Development of non-peptidic inverse agonists of the ghrelin receptor (GHSR) based on the 1,2,4-triazole scaffold
}

Khoubaib Ben Haj Salah, Mathieu Maingot, Anne-Laure Blayo, Céline M'Kadmi, Marjorie Damian, Sophie Mary, Sonia Cantel, Jérémie Neasta, Catherine Oiry, Sylvie Péraldi-Roux, Gimena Fernandez, Guadalupe Garcia Romero, Mario Perello, Jacky Marie, Jean-Louis Banères, Jean-Alain Fehrentz, Séverine Denoyelle*

Table of Contents:

1. Copies of ${ }^{1} \mathrm{H}$ and ${ }^{13} \mathrm{C}$ NMR spectra of all final ligands 1-46

2. LC-MS chromatograms of key target ligands 27, 29, 30 and 44

3. Determination of the water solubility of final ligand $\mathbf{2 7}$

4. Determination of the water solubility of final ligand $\mathbf{2 9}$

5. Determination of the water solubility of final ligand $\mathbf{3 0}$

6. Determination of the water solubility of final ligand $\mathbf{4 4}$ 
1. Copies of ${ }^{1} \mathrm{H}$ and ${ }^{13} \mathrm{C}$ NMR spectra of all final ligands 1-46

Compound 1 (JMV2959)
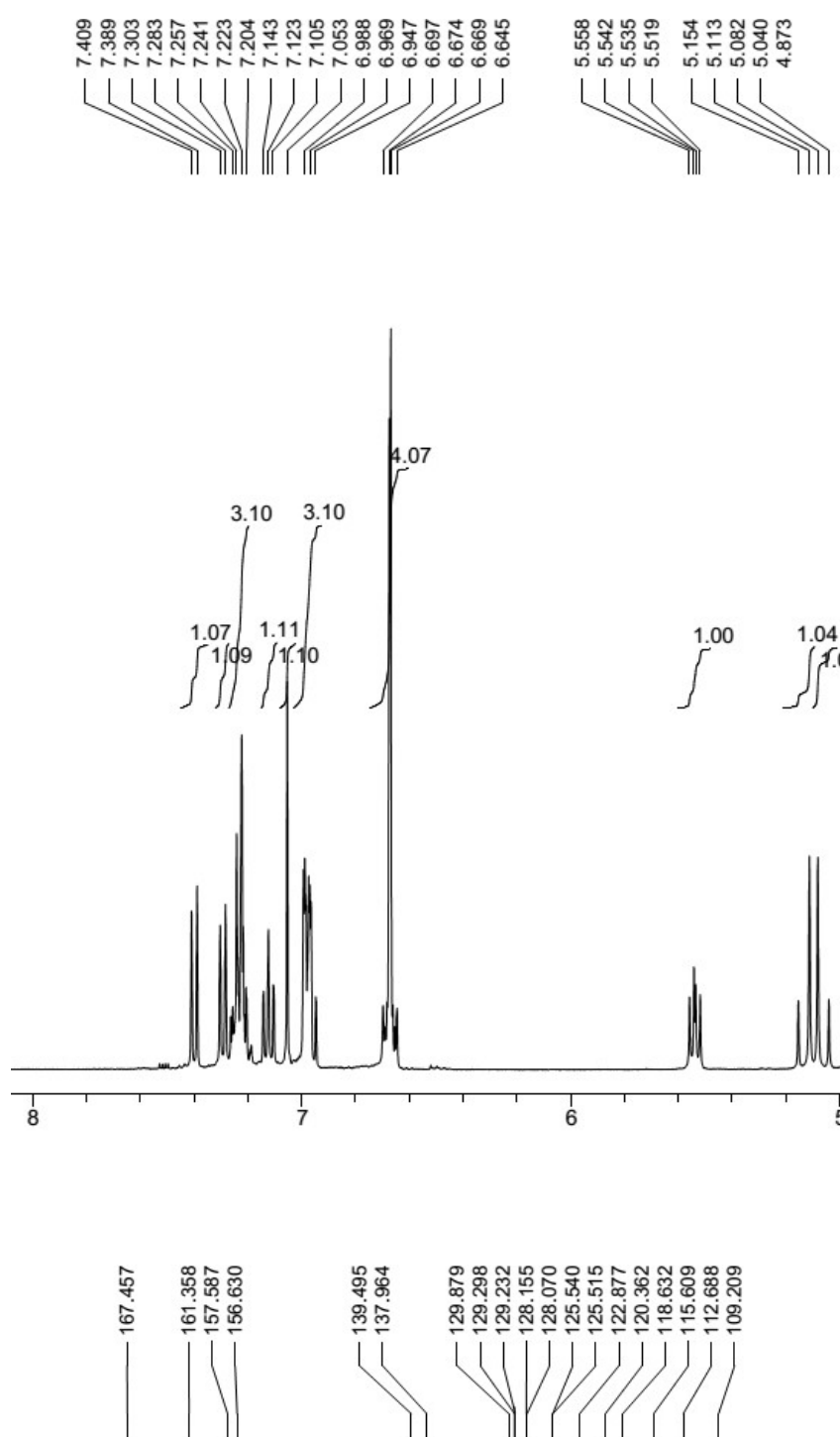

$\iiint^{1.04}$
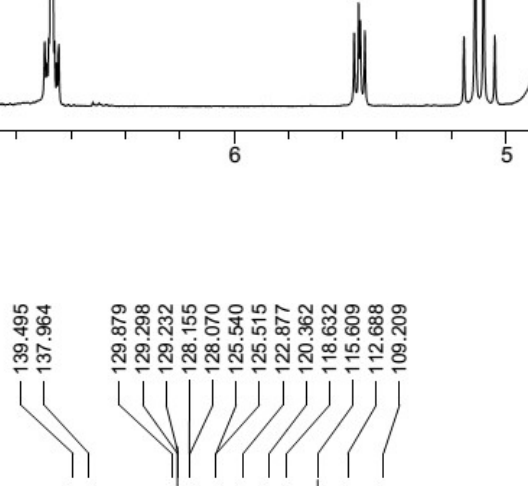

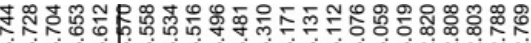

(1)
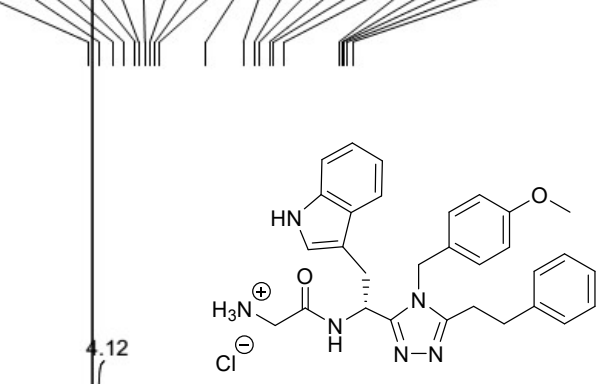

$\int^{2.09} \int^{2.00} \int^{2.10}$

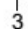

PPM
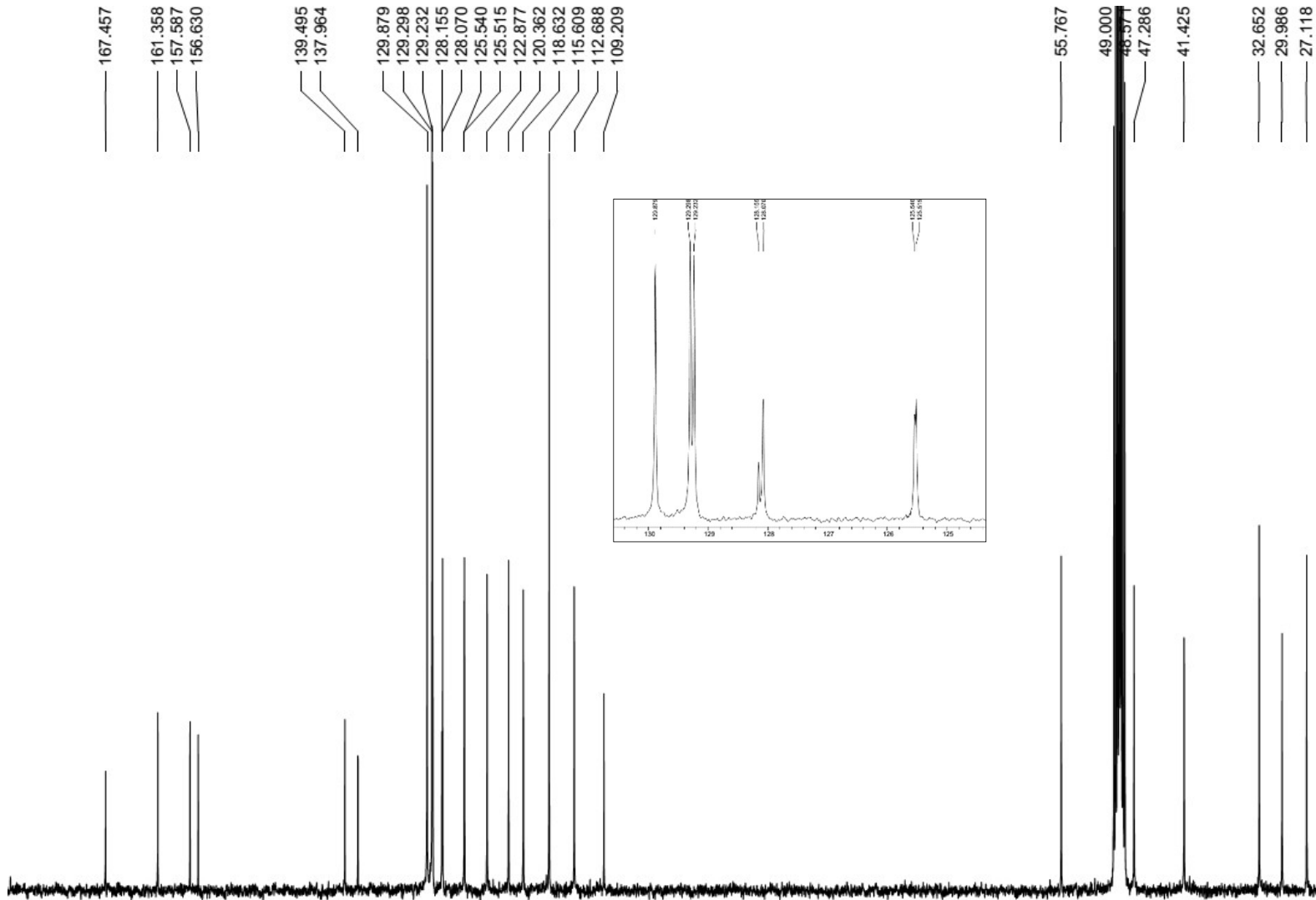

160 
Compound 2
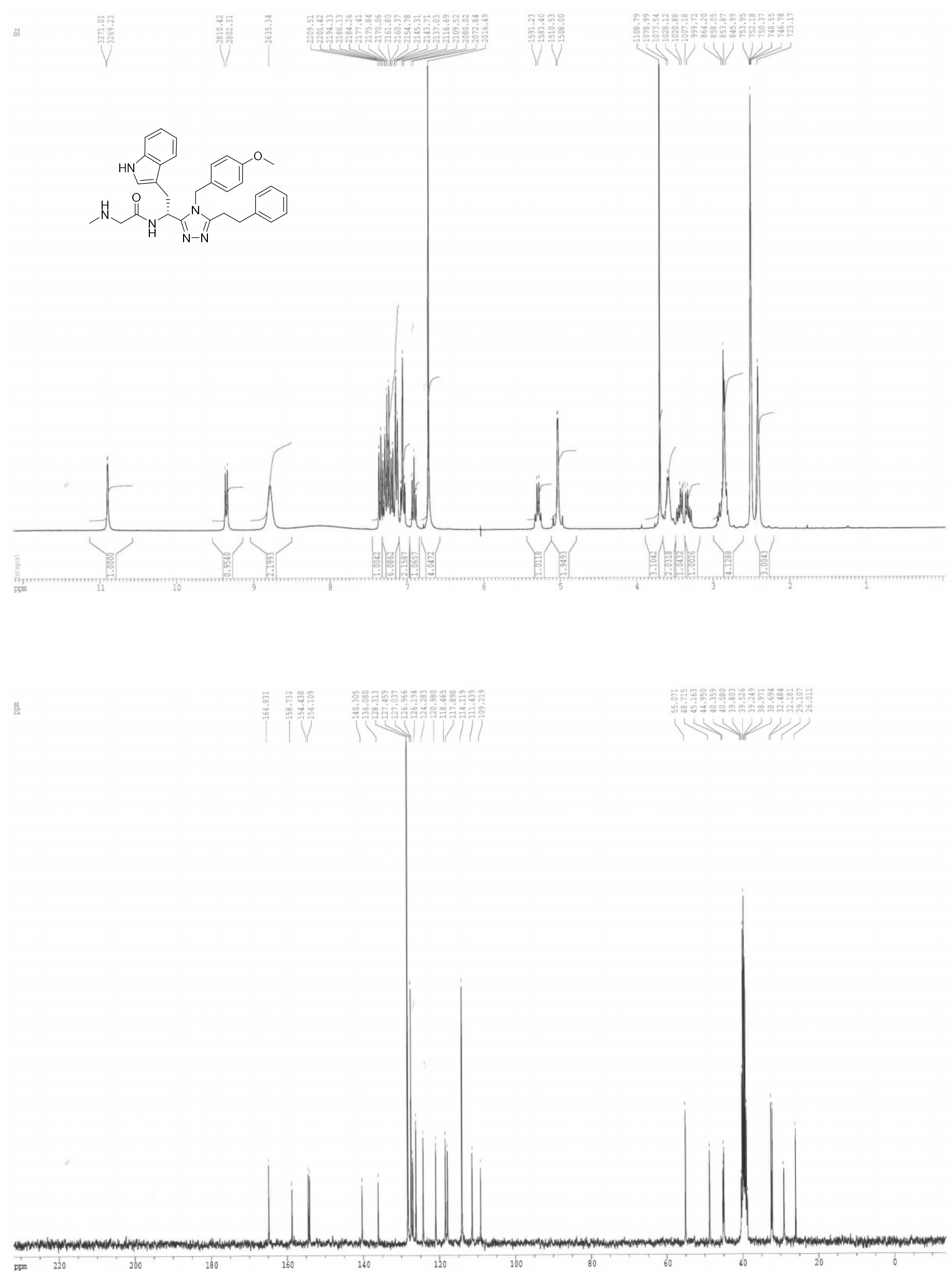
Compound 3

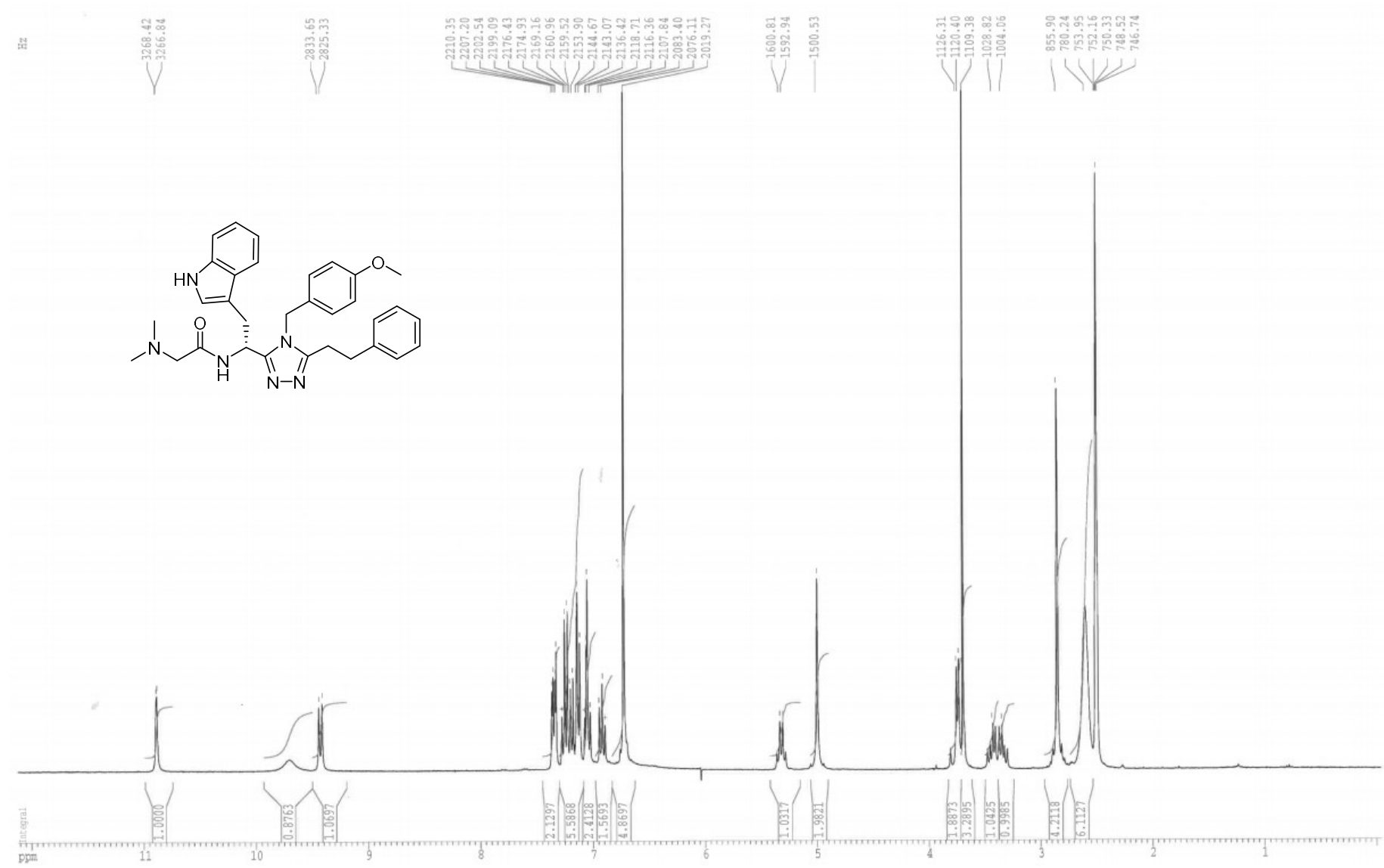

喜

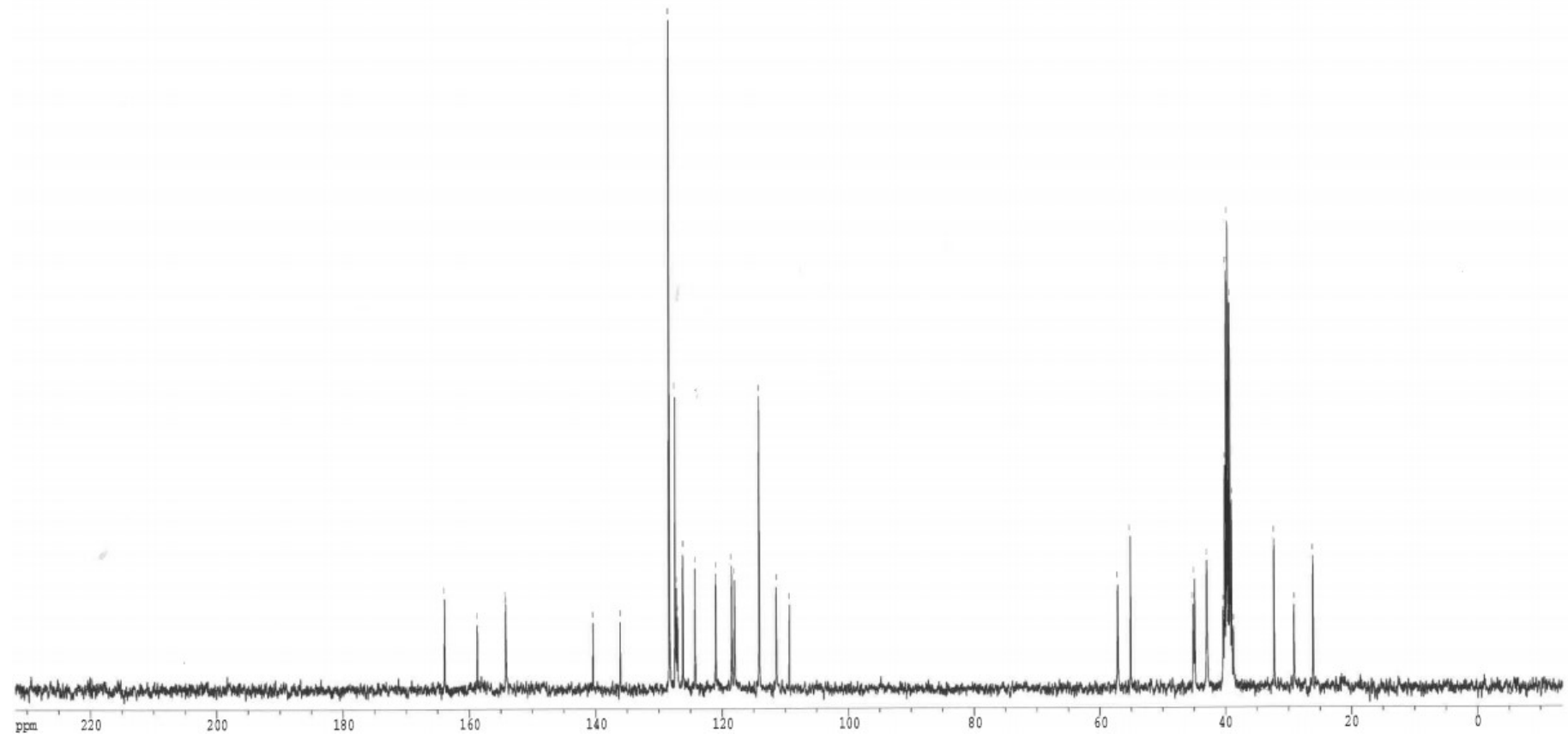


Compound 4

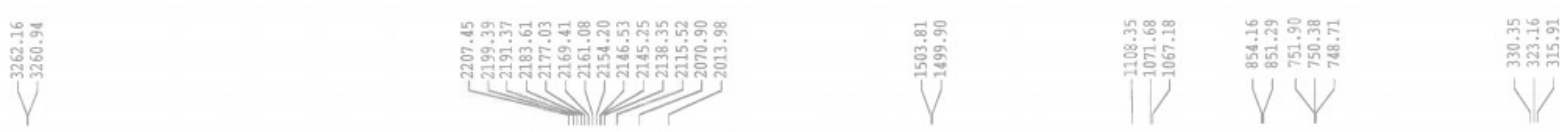
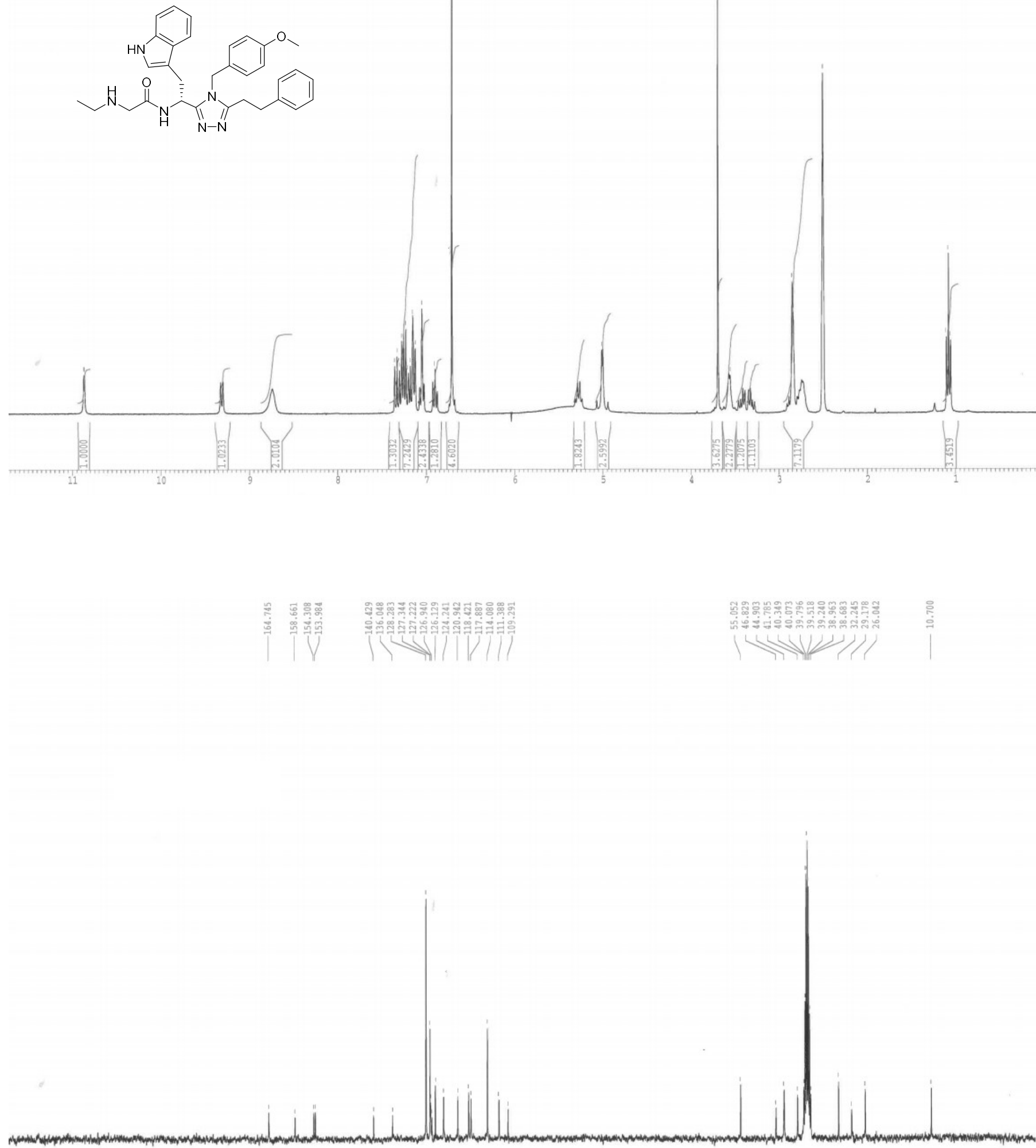
Compound 5
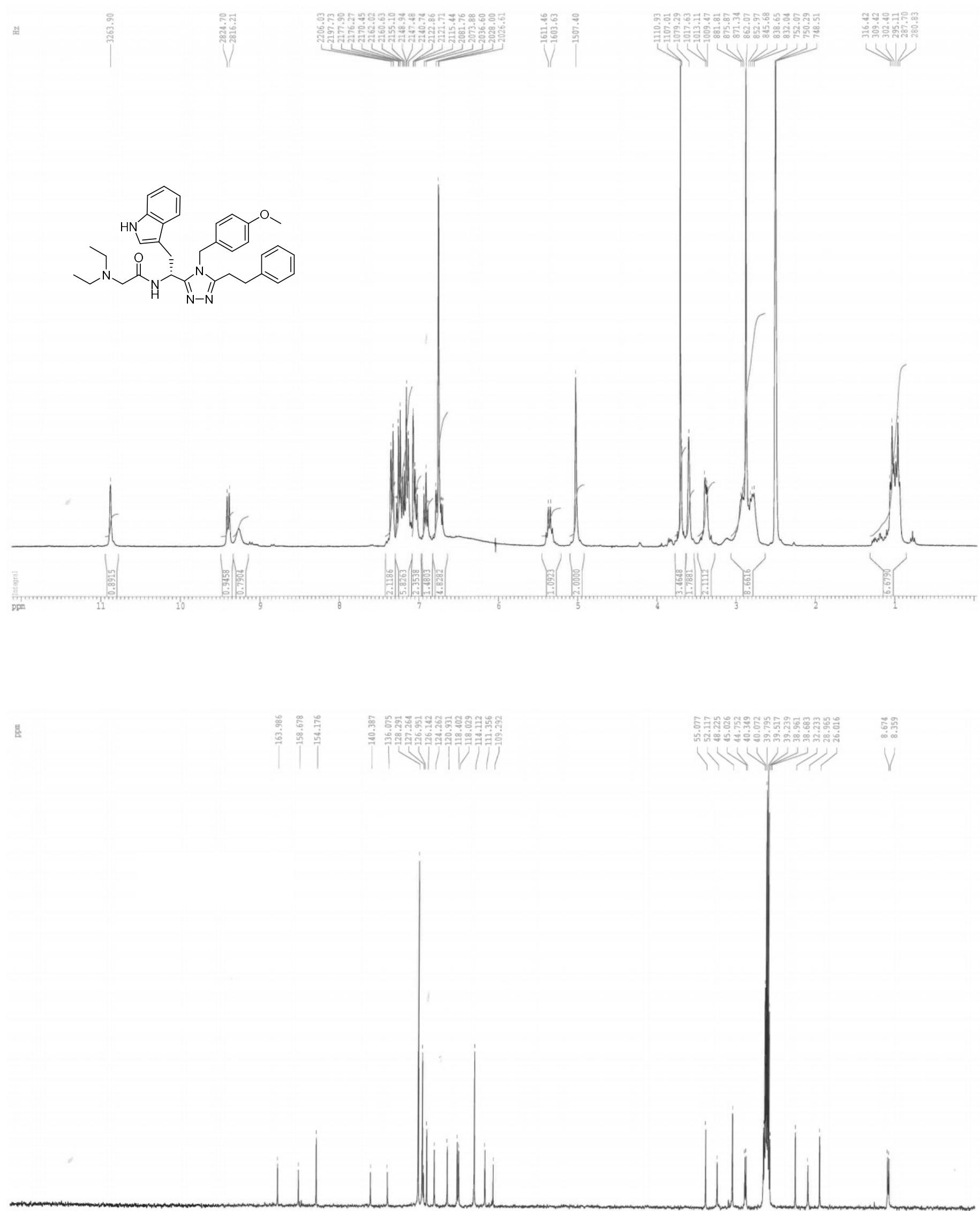

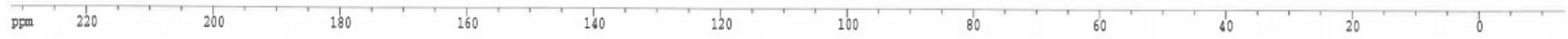


Compound $\mathbf{6}$

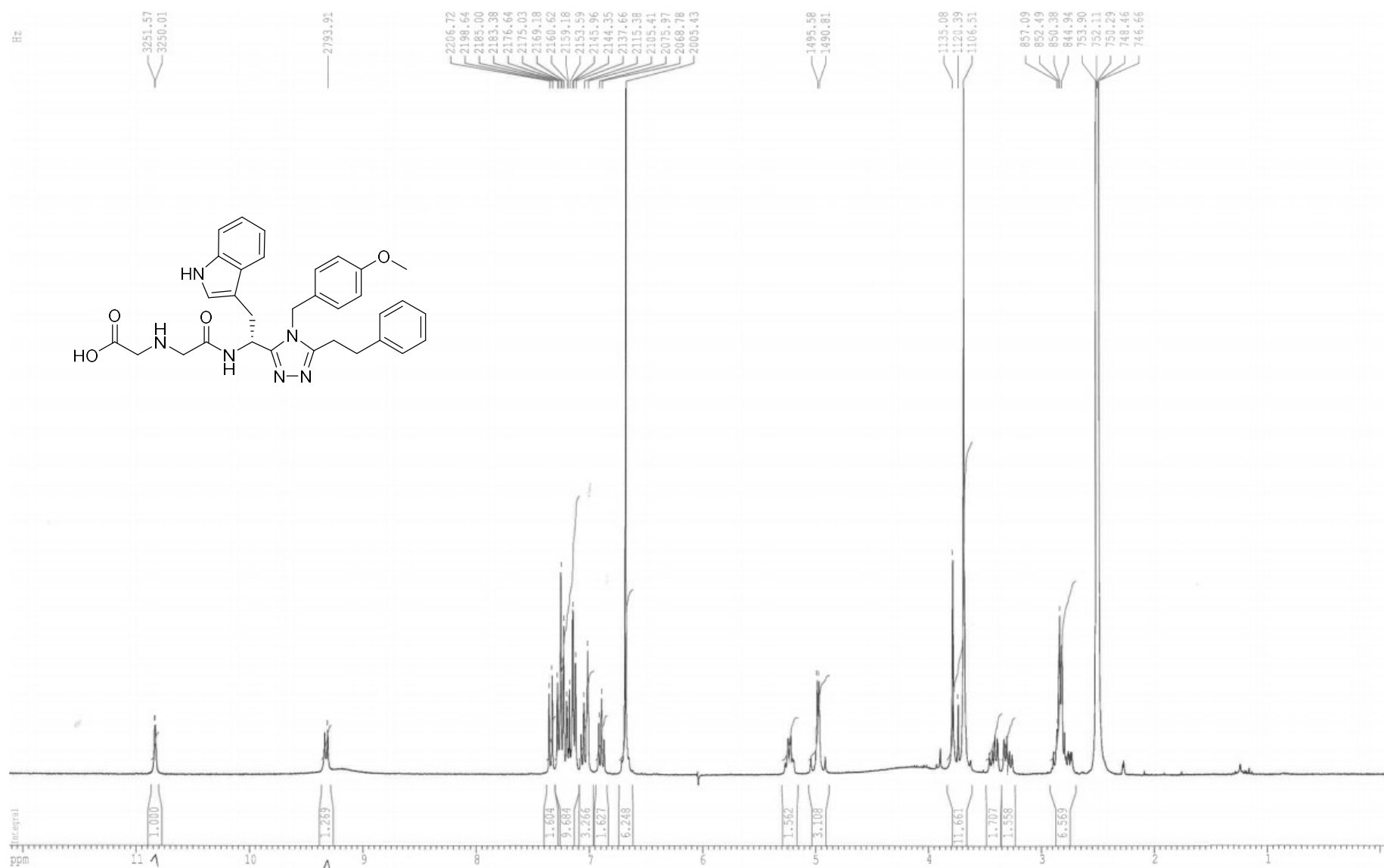

||

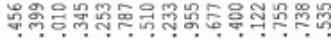

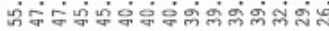

(

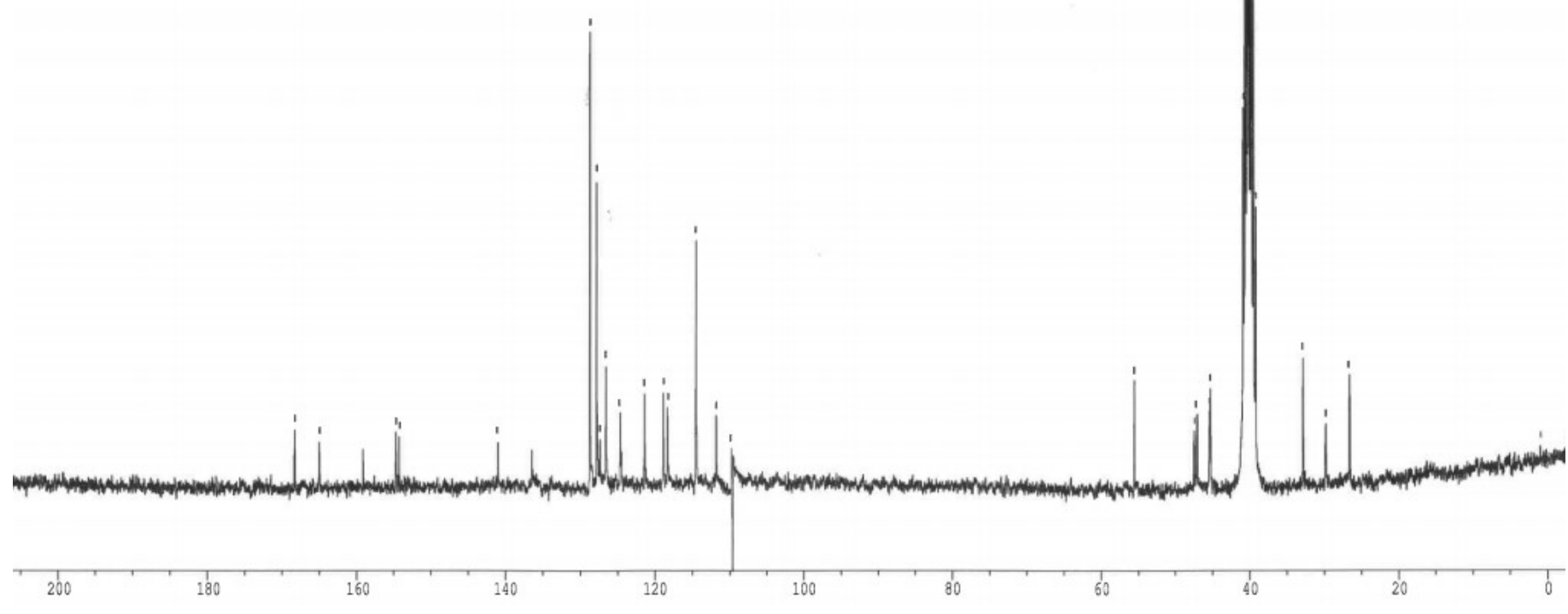


Compound 7
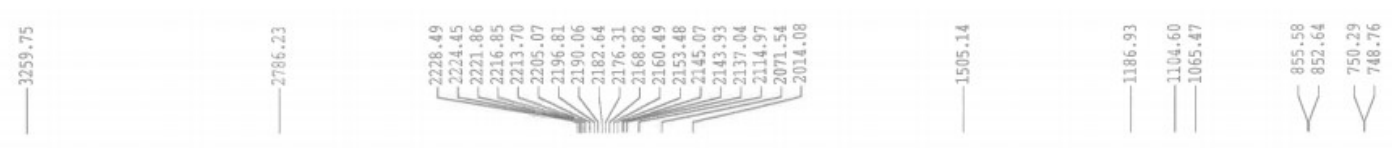<smiles>COc1ccc(Cn2c(CCc3ccccc3)nnc2[C@H](NC(=O)CNCc2ccccc2)c2c[nH]c3ccccc23)cc1</smiles>
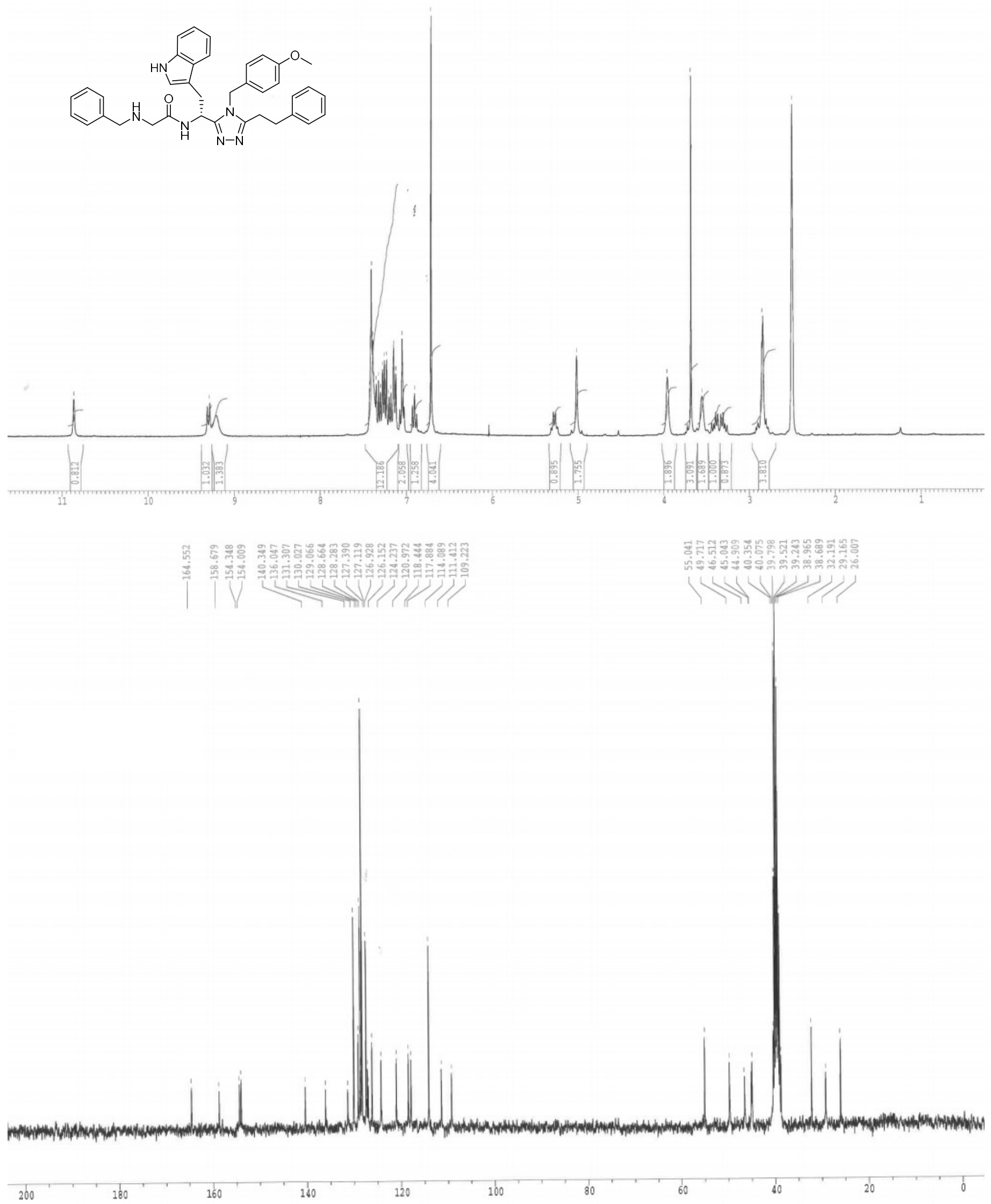

So 
Compound 8

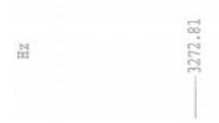

ํำ
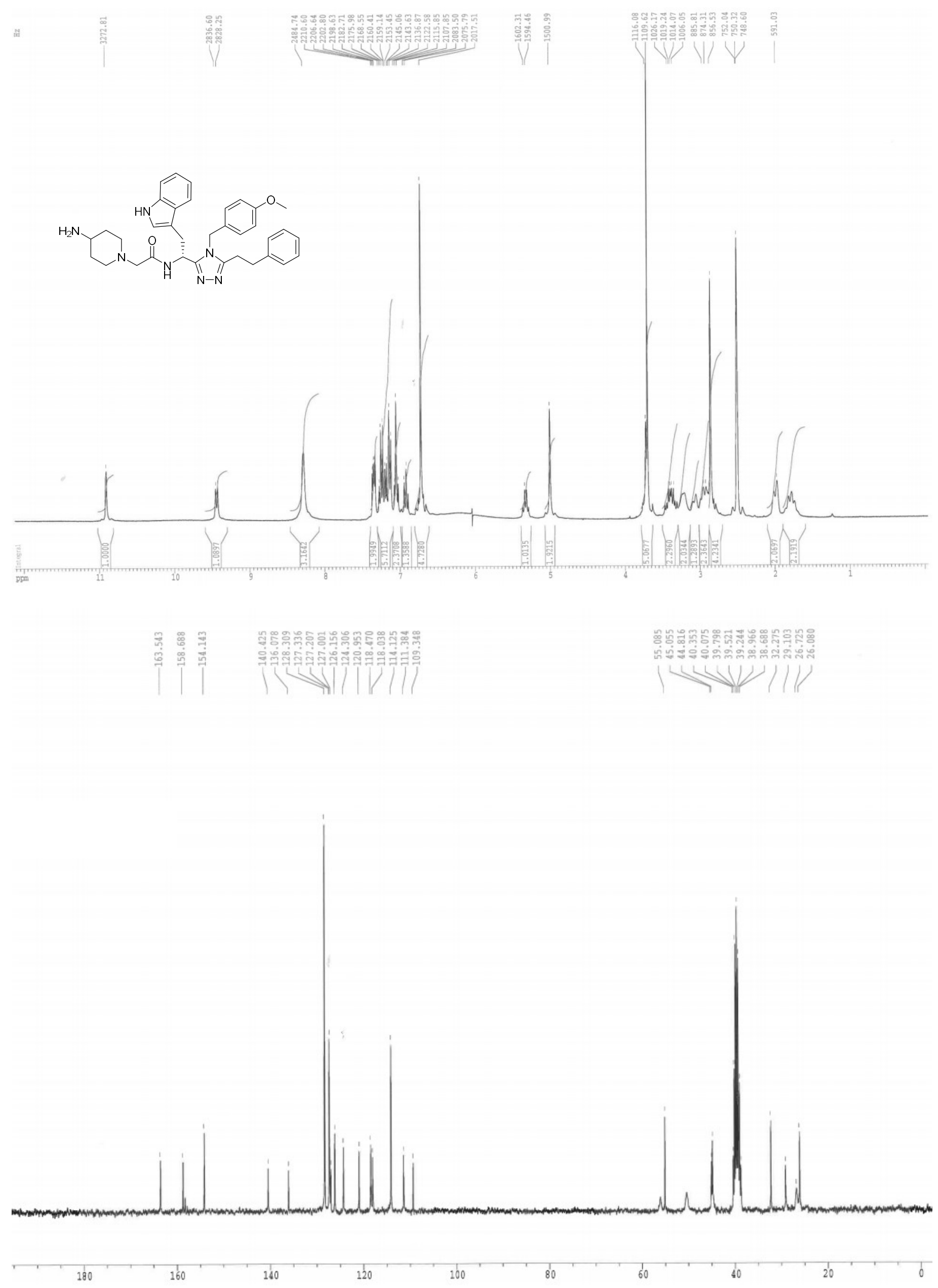

S9 


\section{Compound 9}
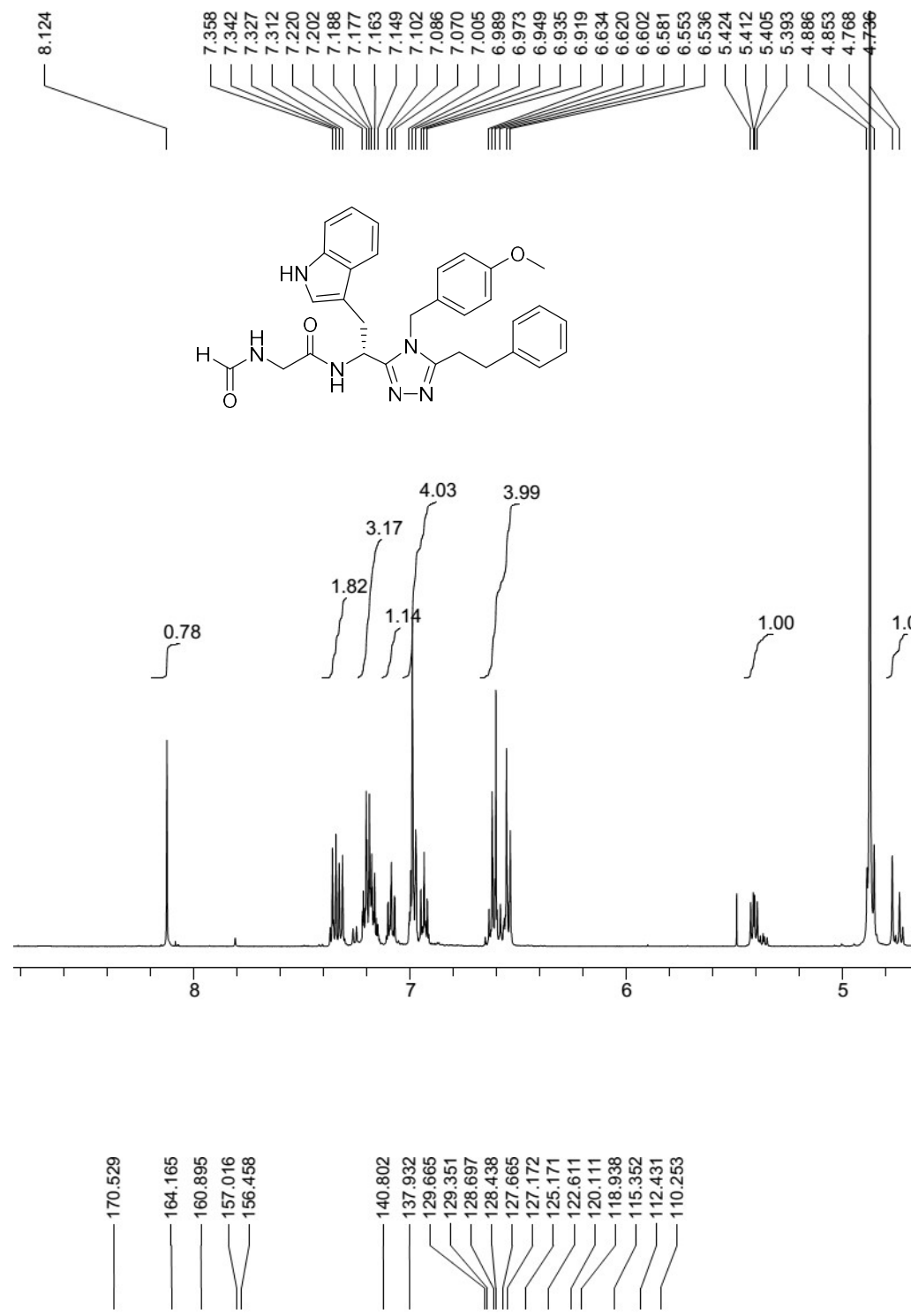

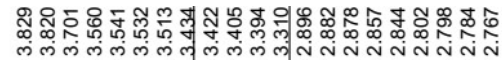
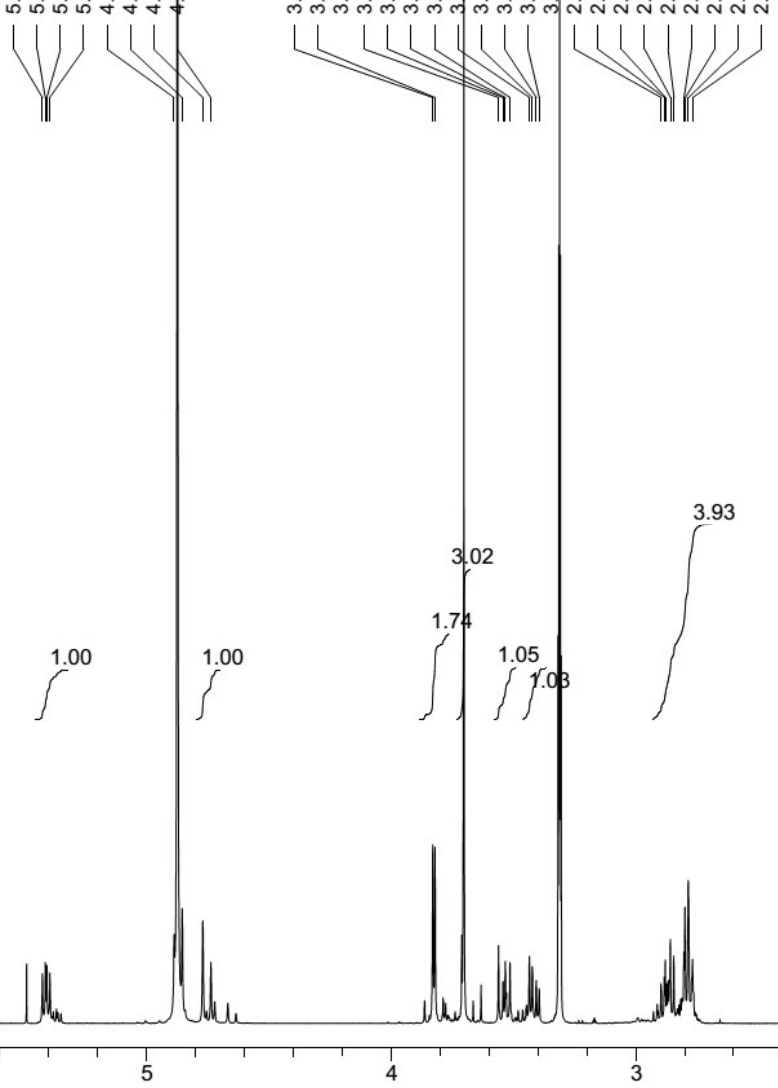

3

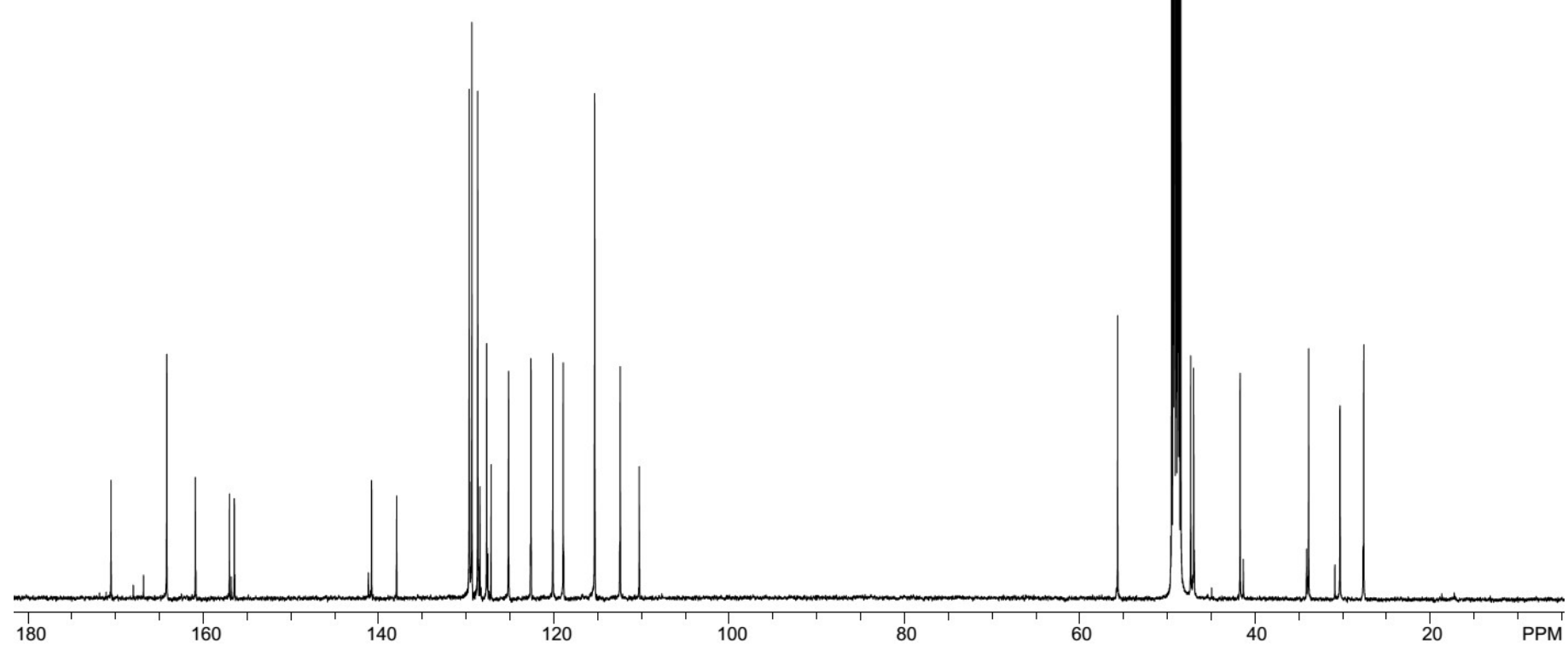




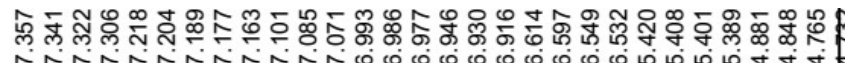

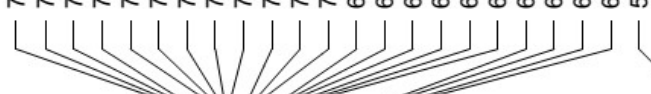

and || I|
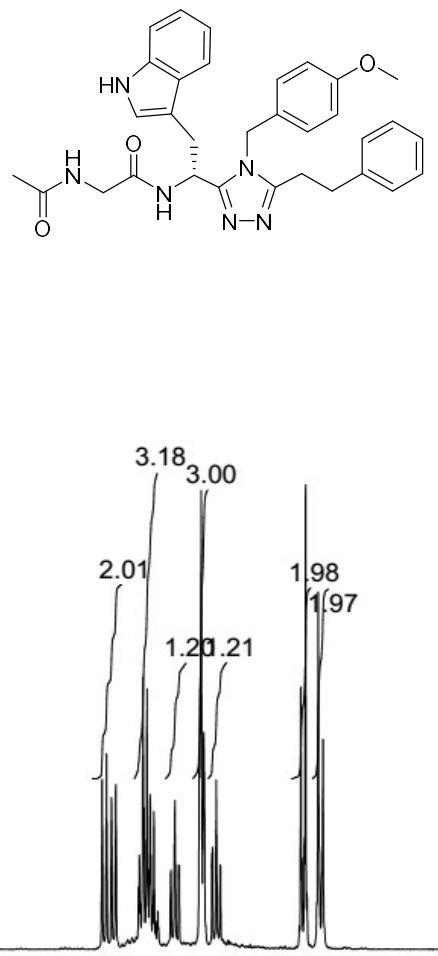

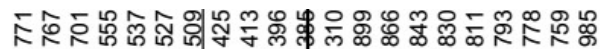

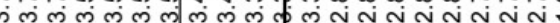
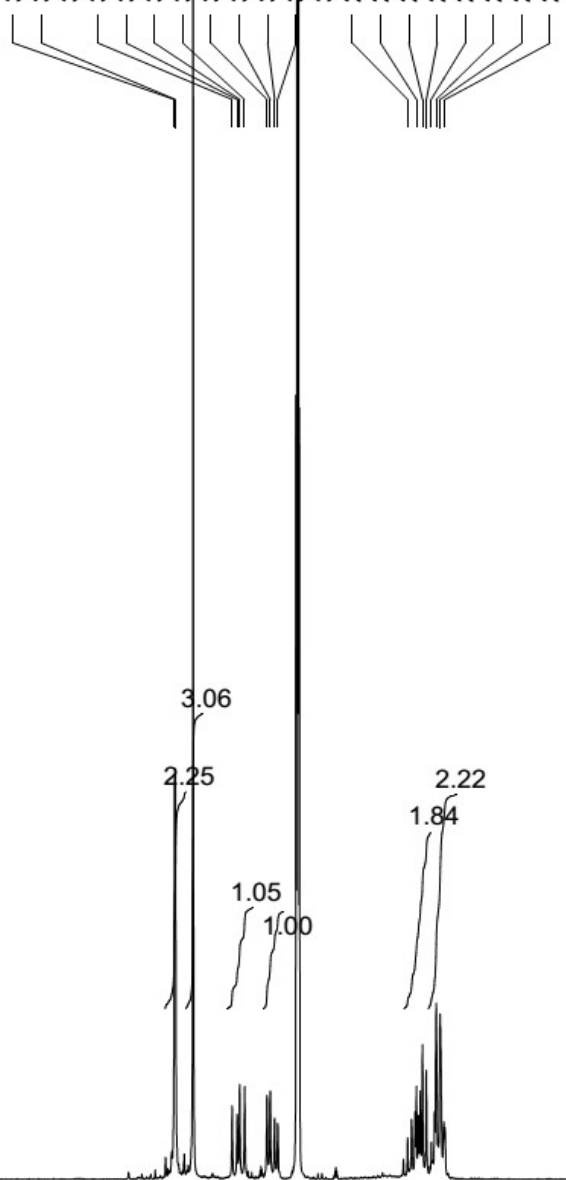
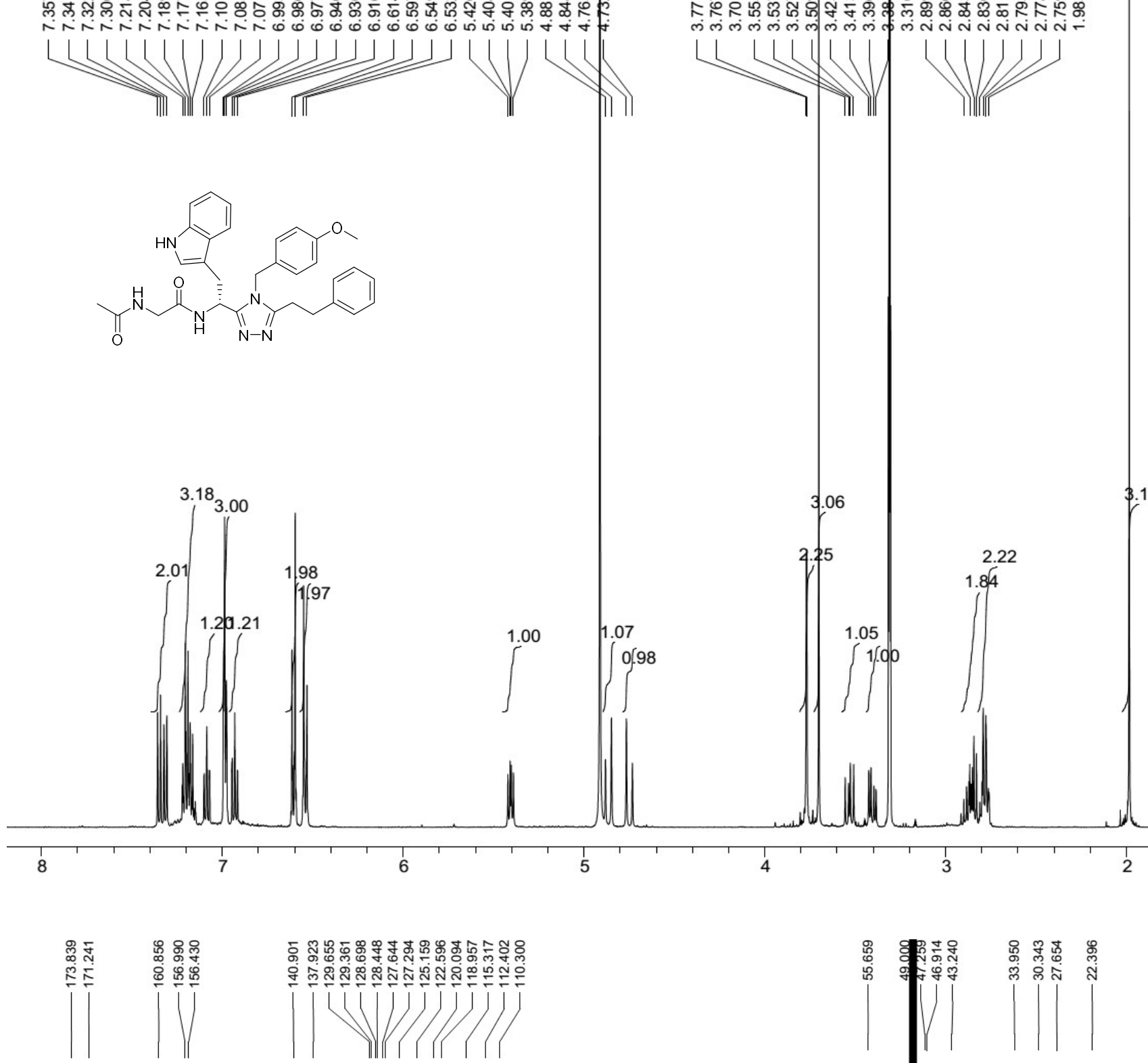

6

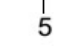

3

2
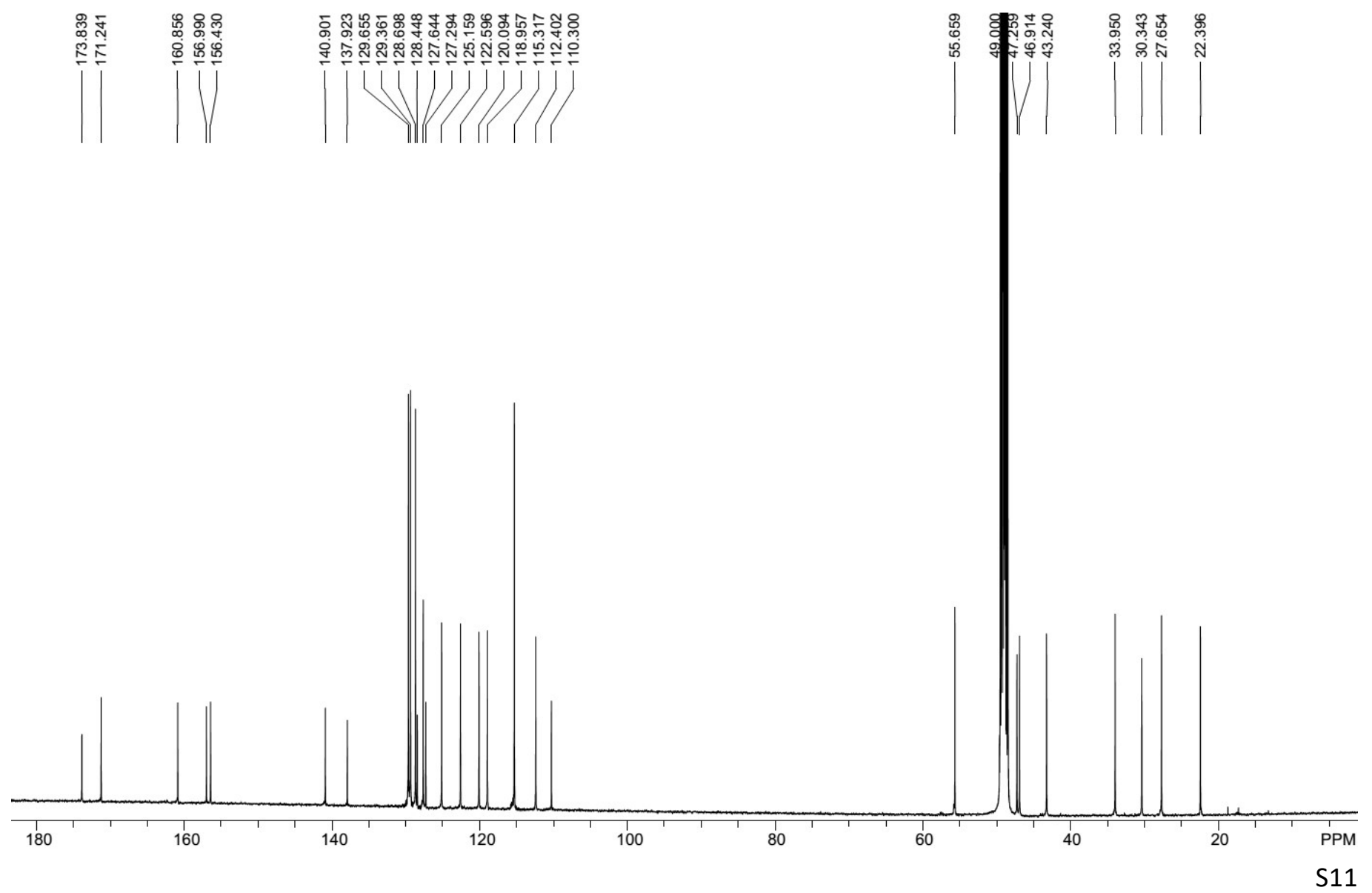


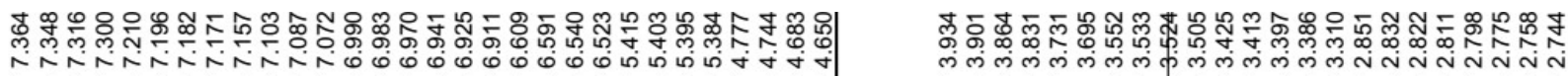

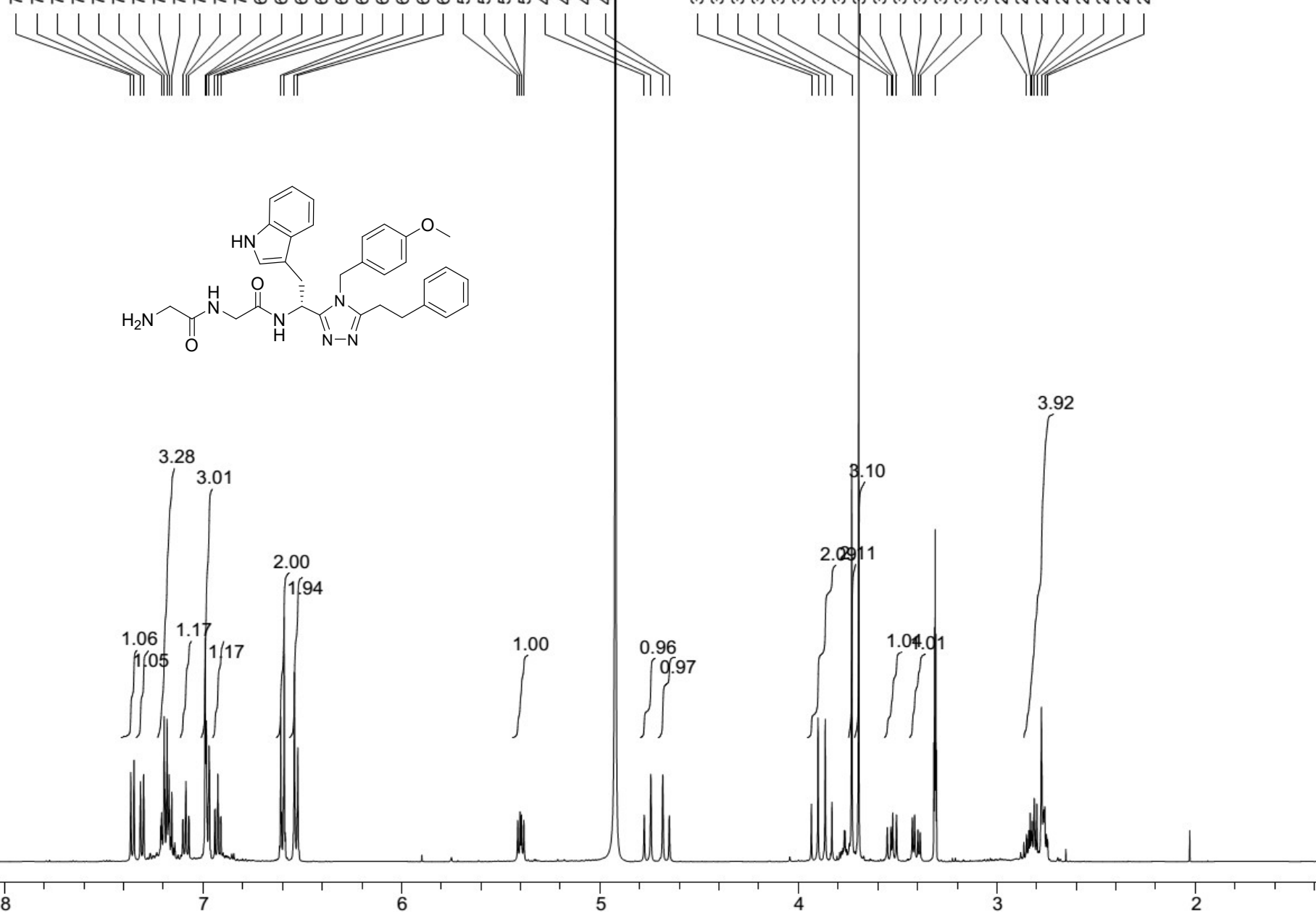

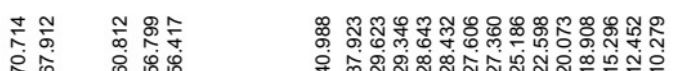

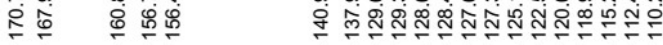

II if II Willill

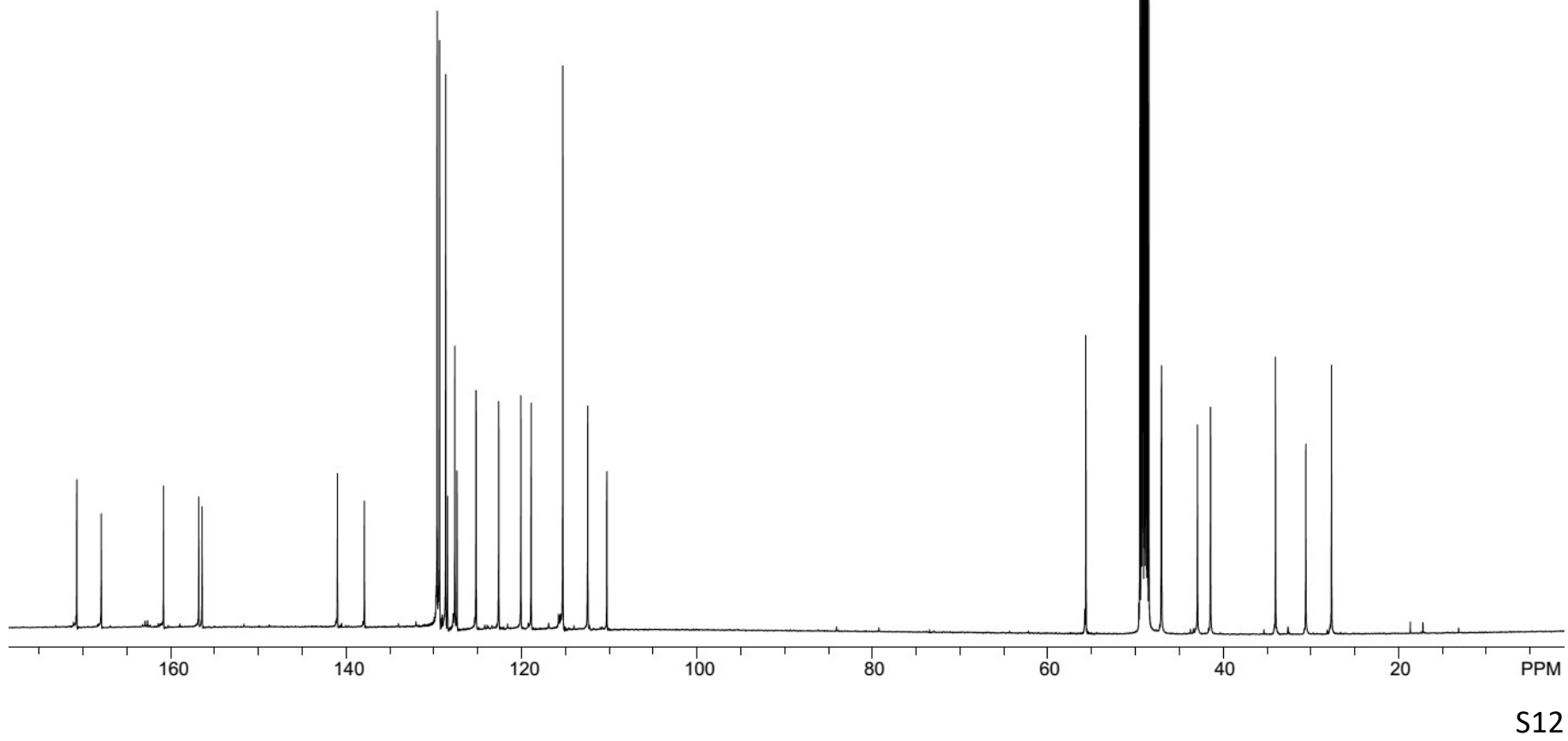



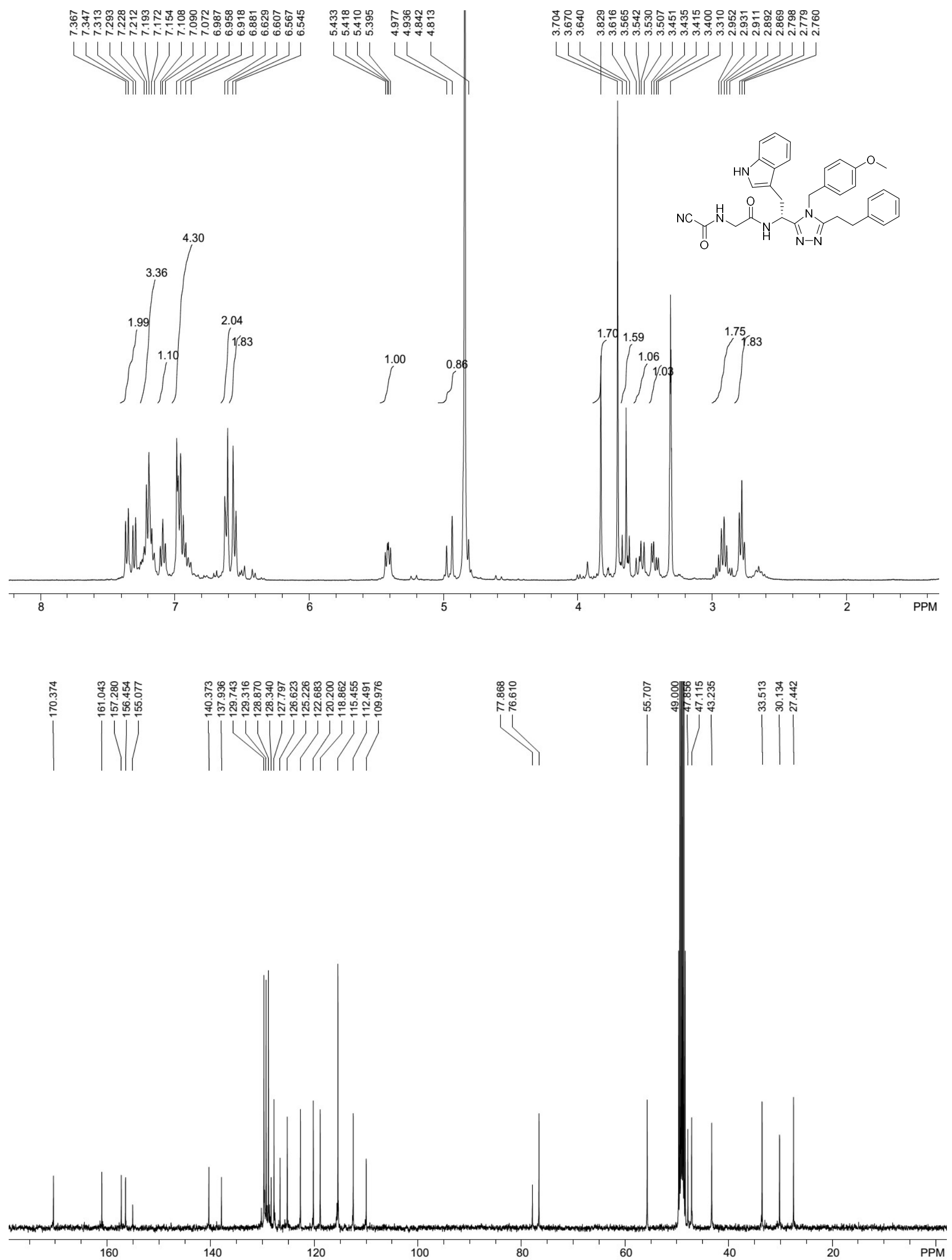

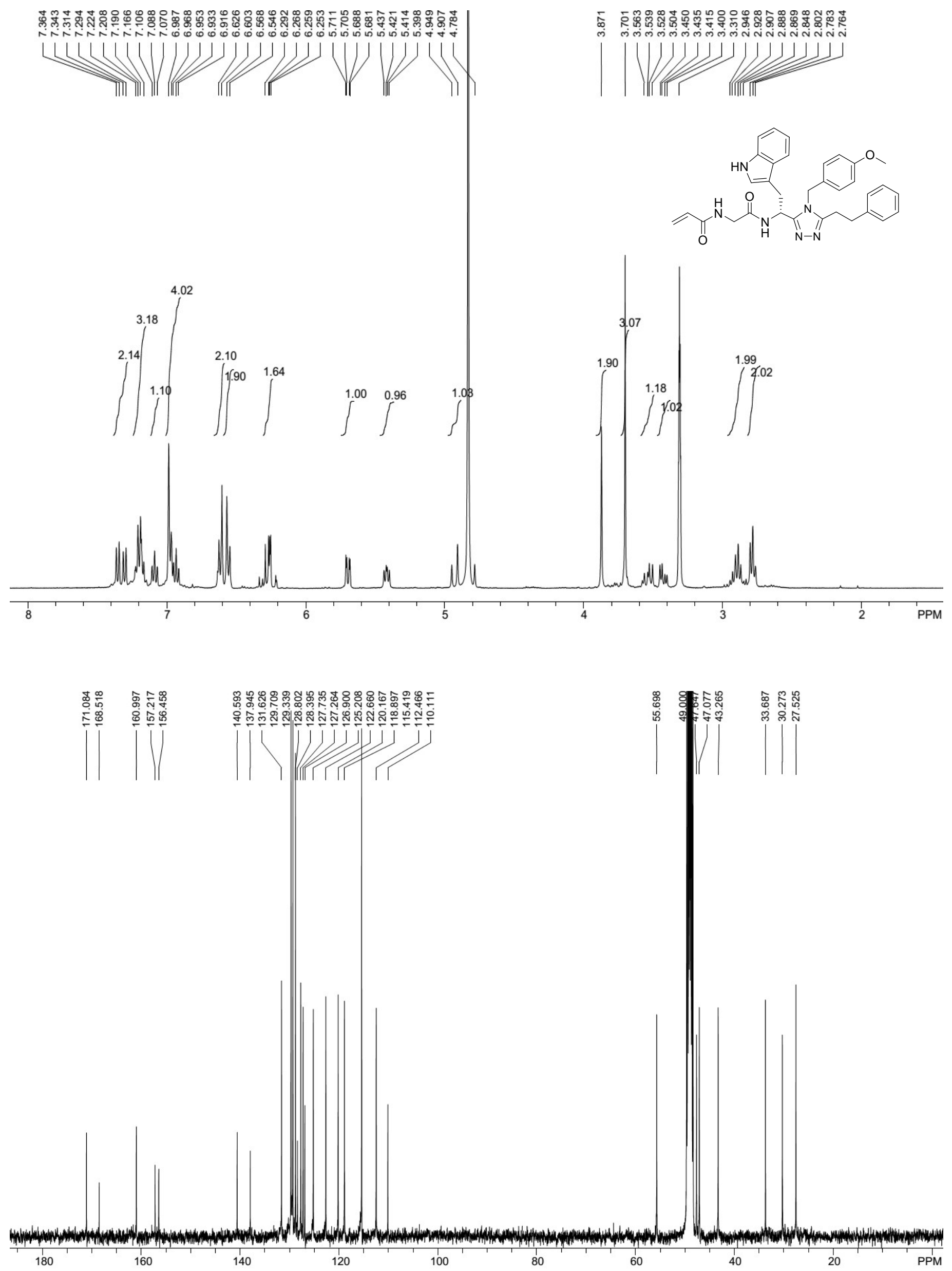


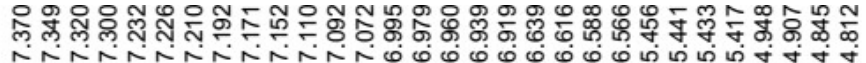

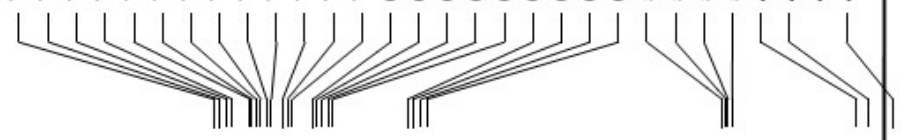

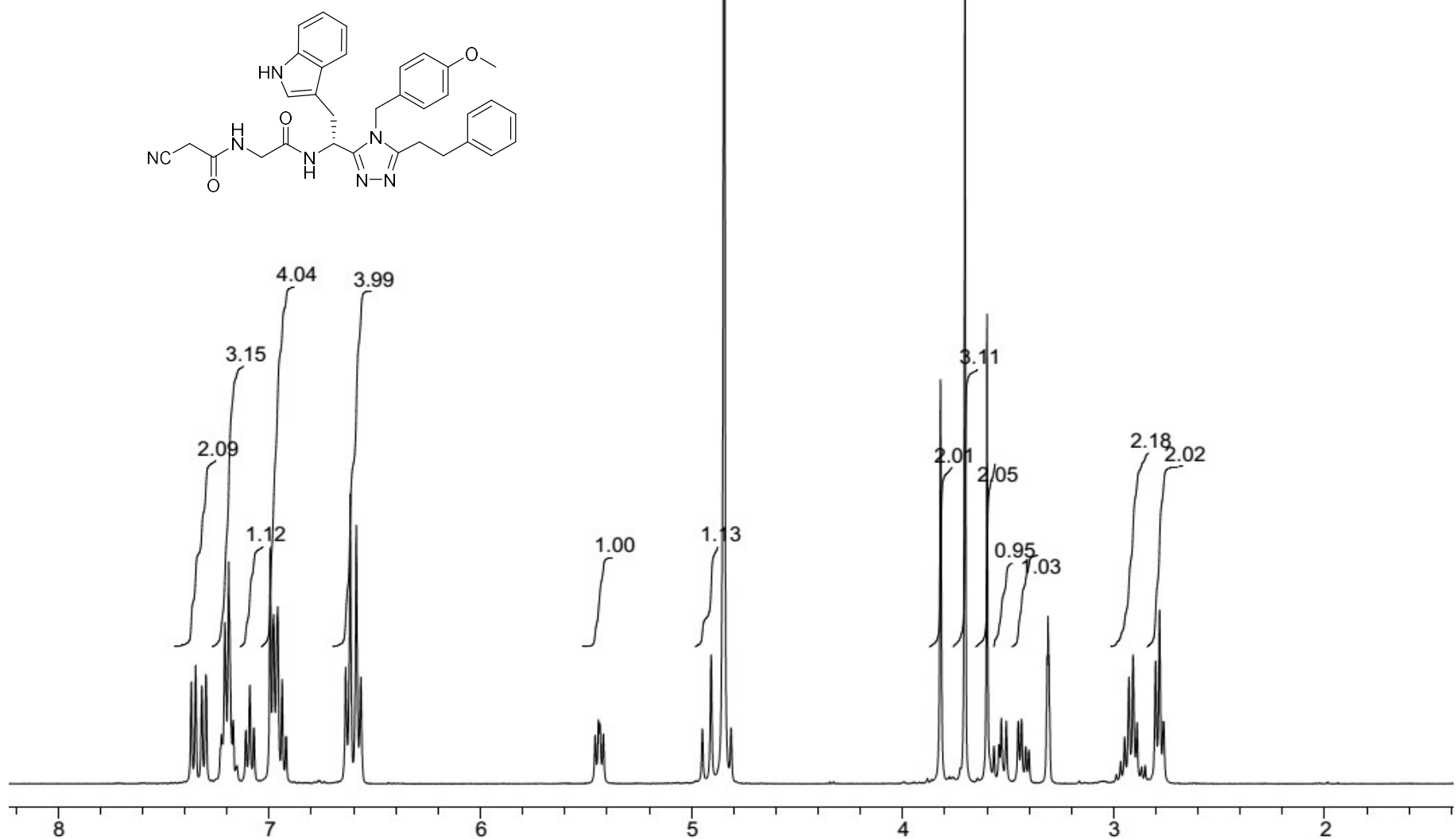

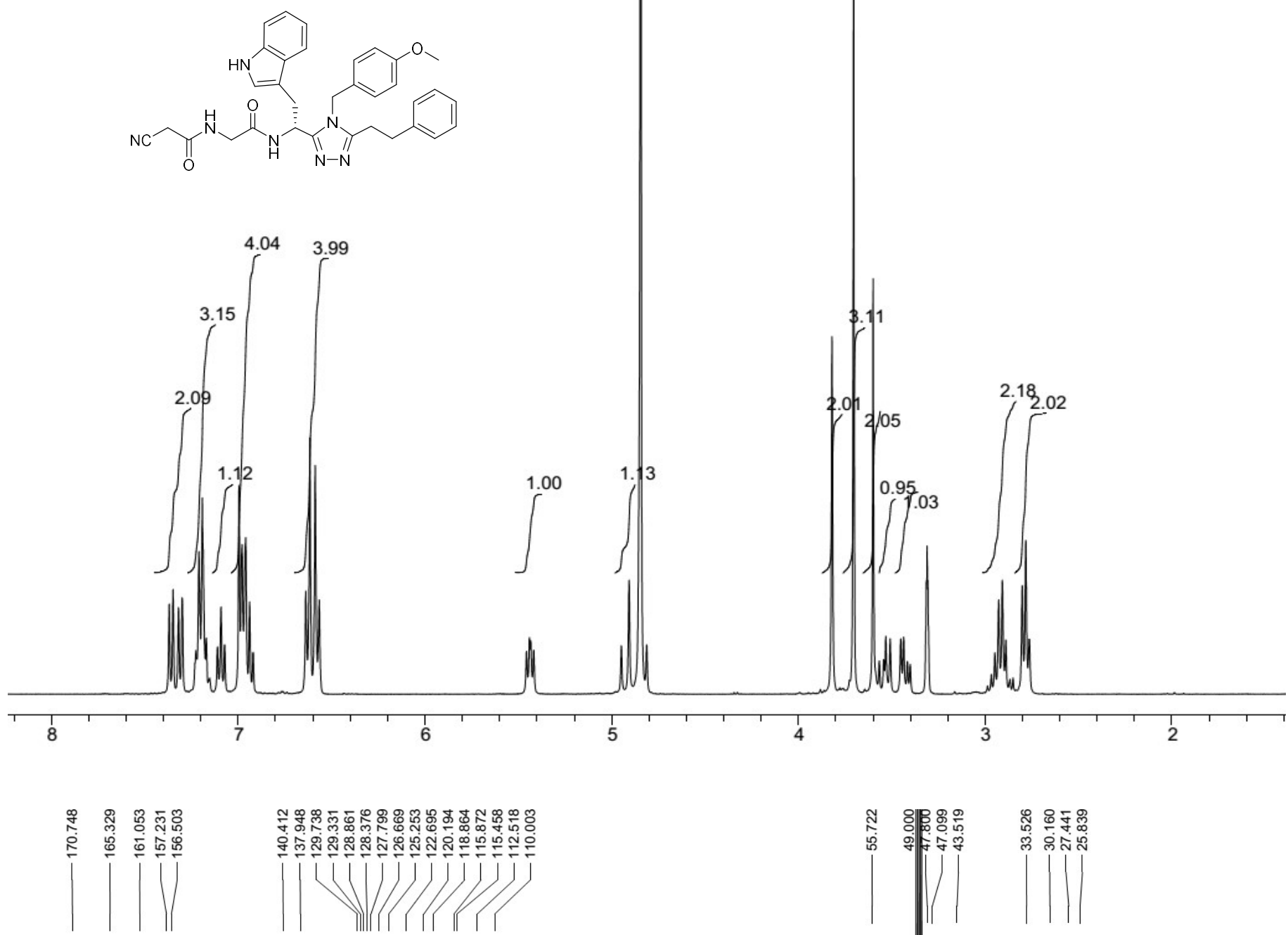

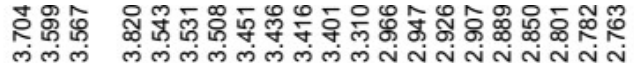

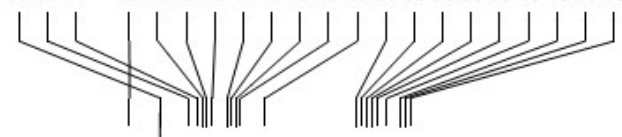

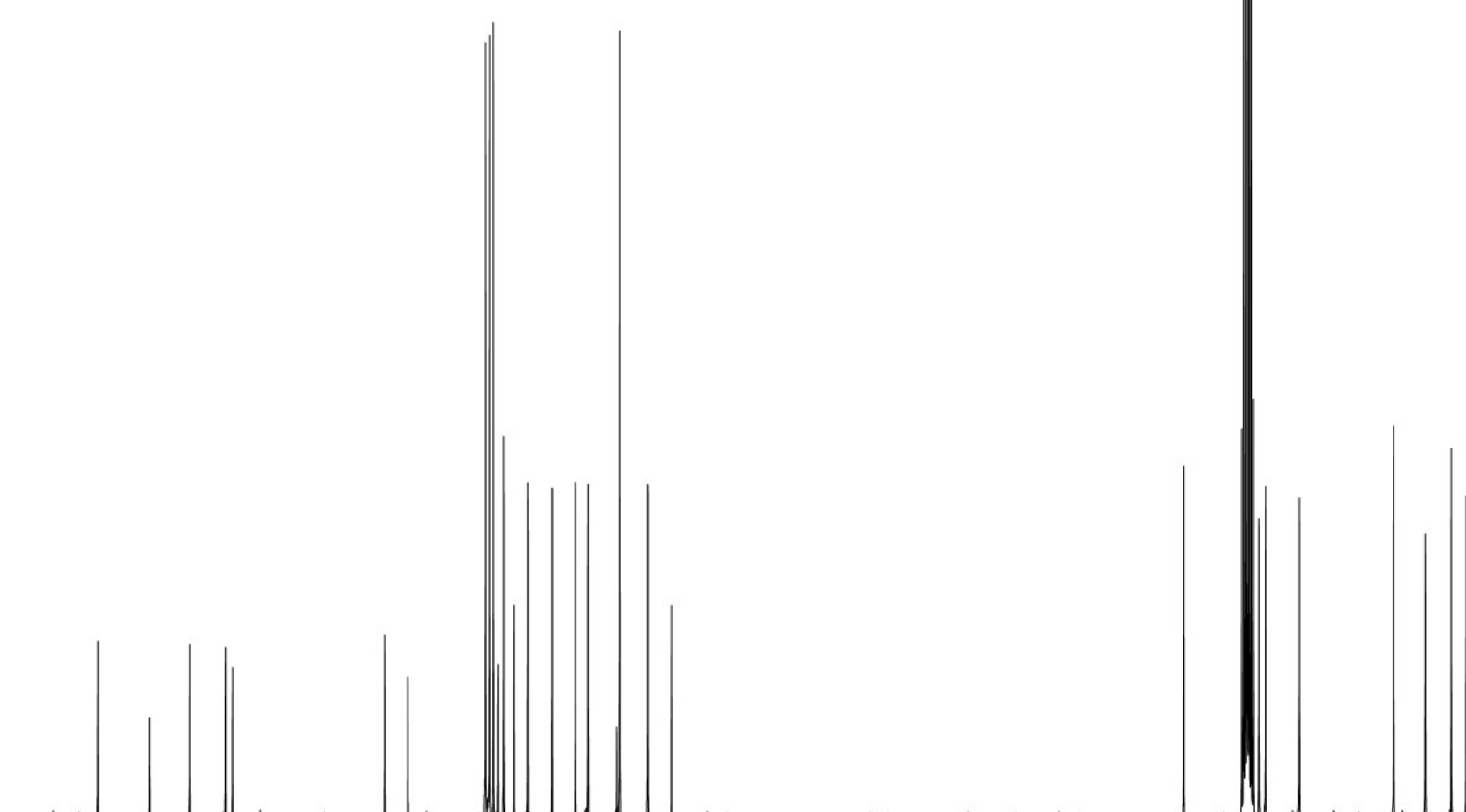




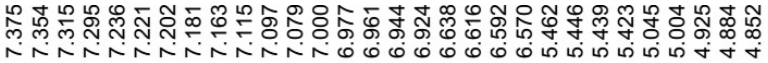

U4

ain

|| || ||||| ||<smiles></smiles>

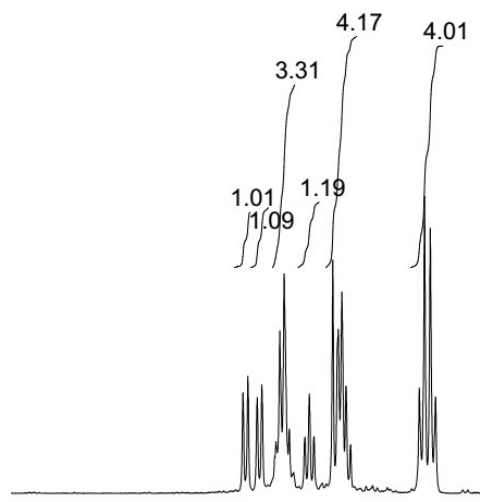

8

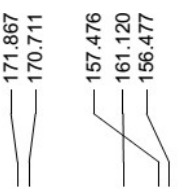

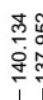

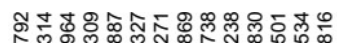

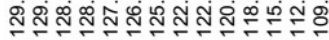

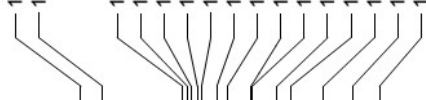

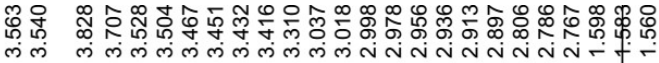

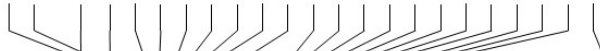

in
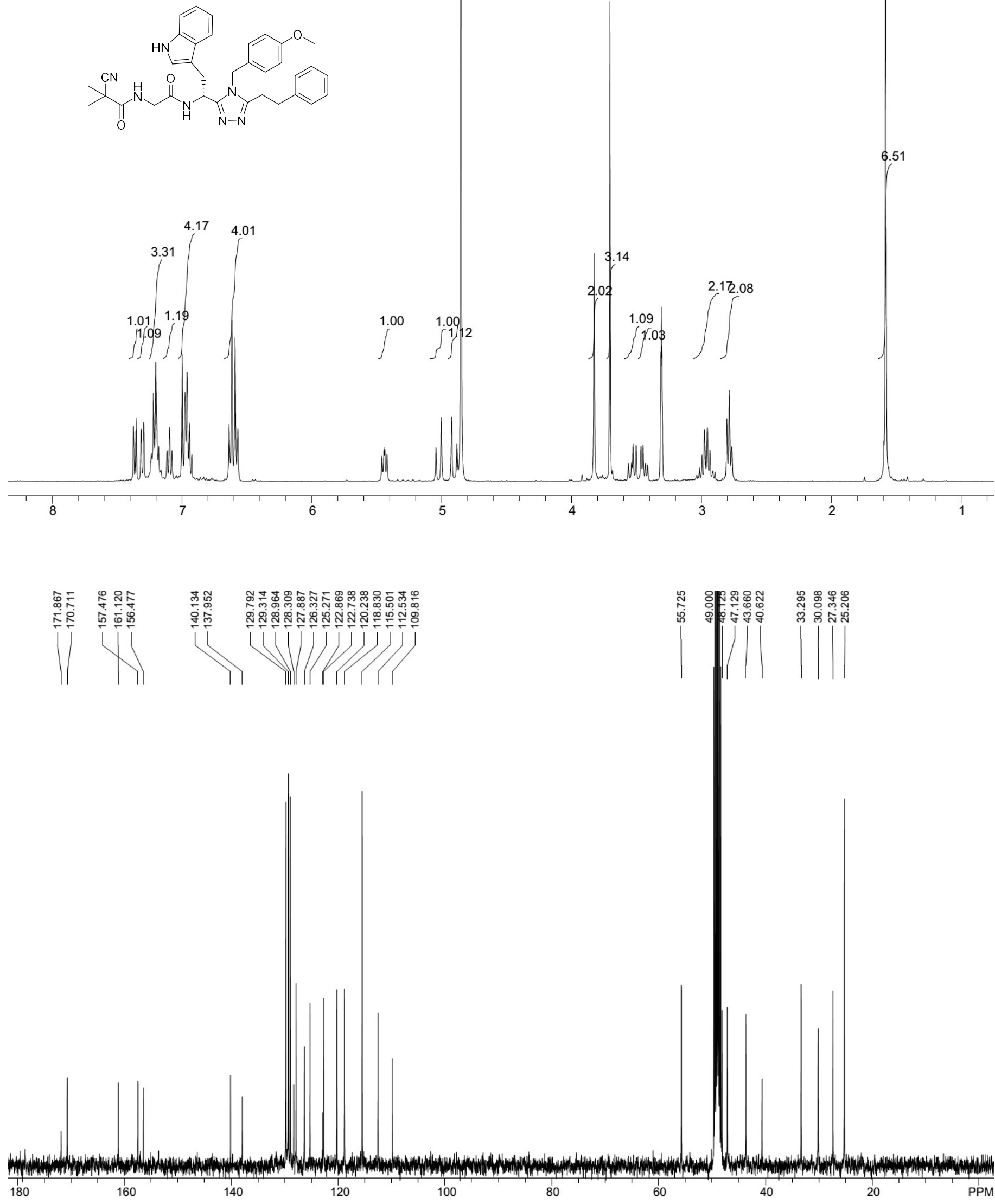


\section{Compound 16}

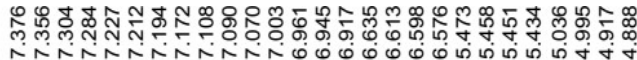
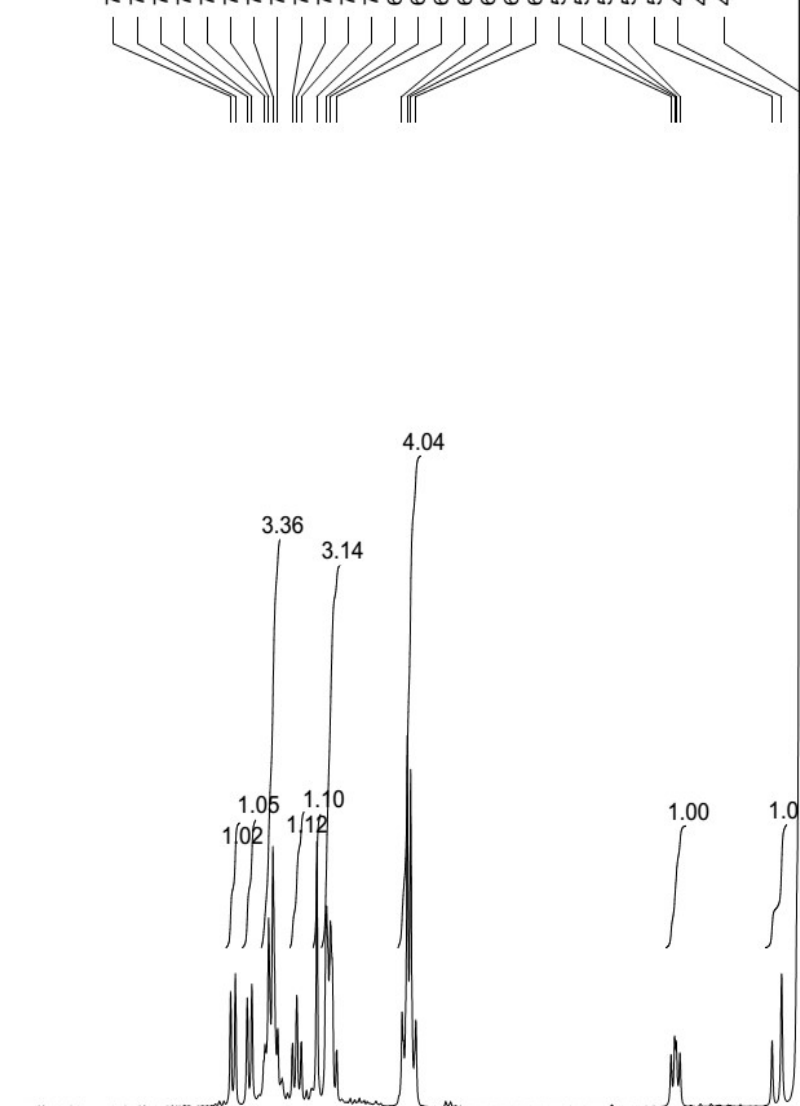

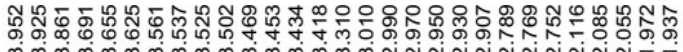

Lom

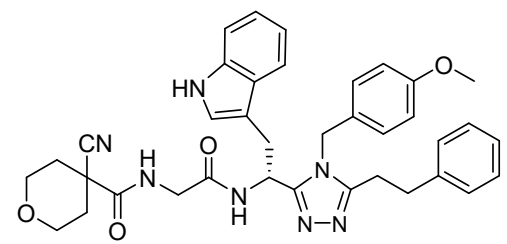

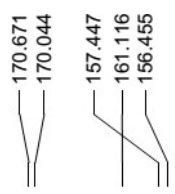
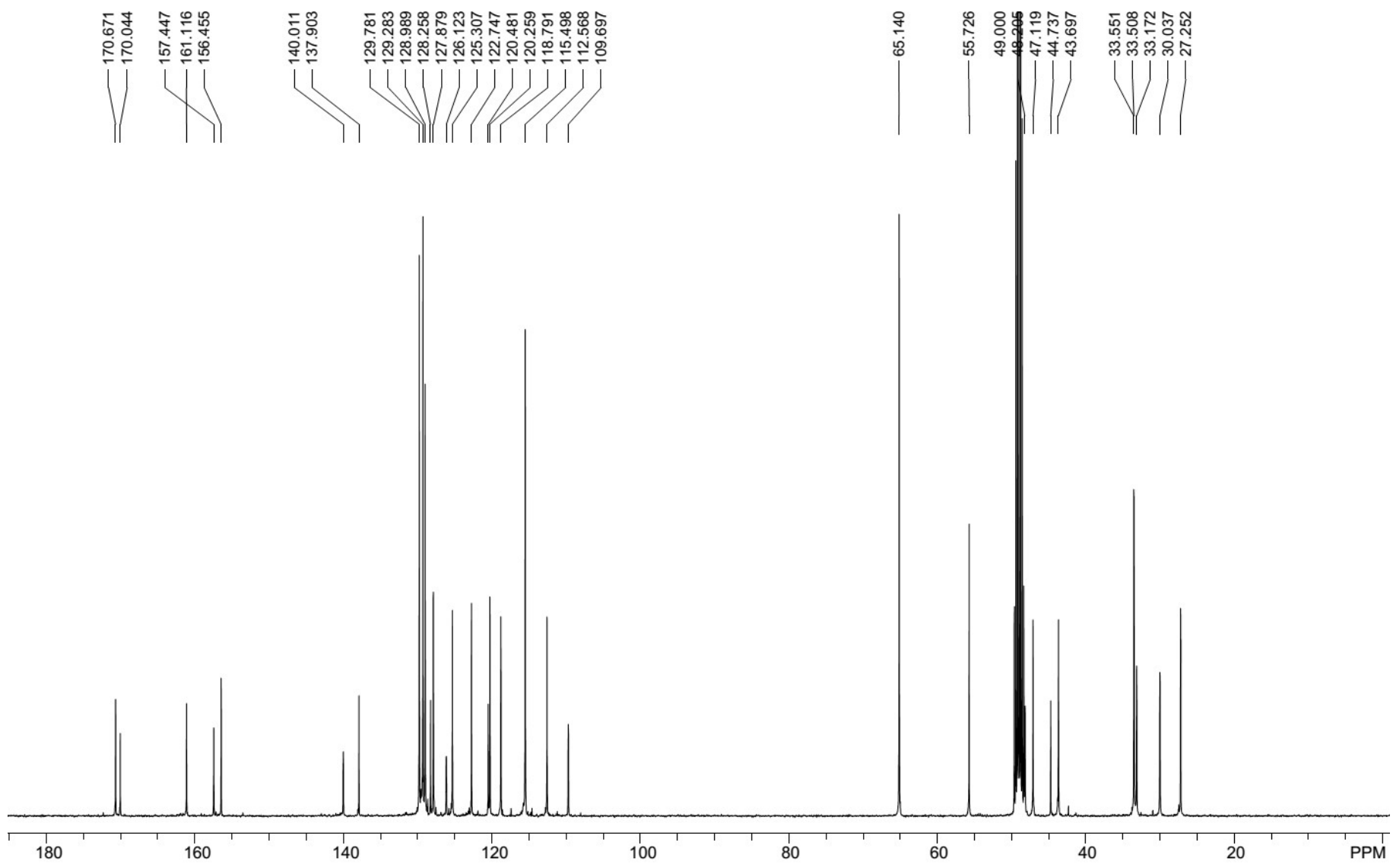


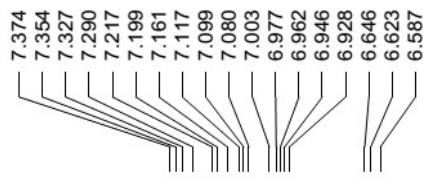
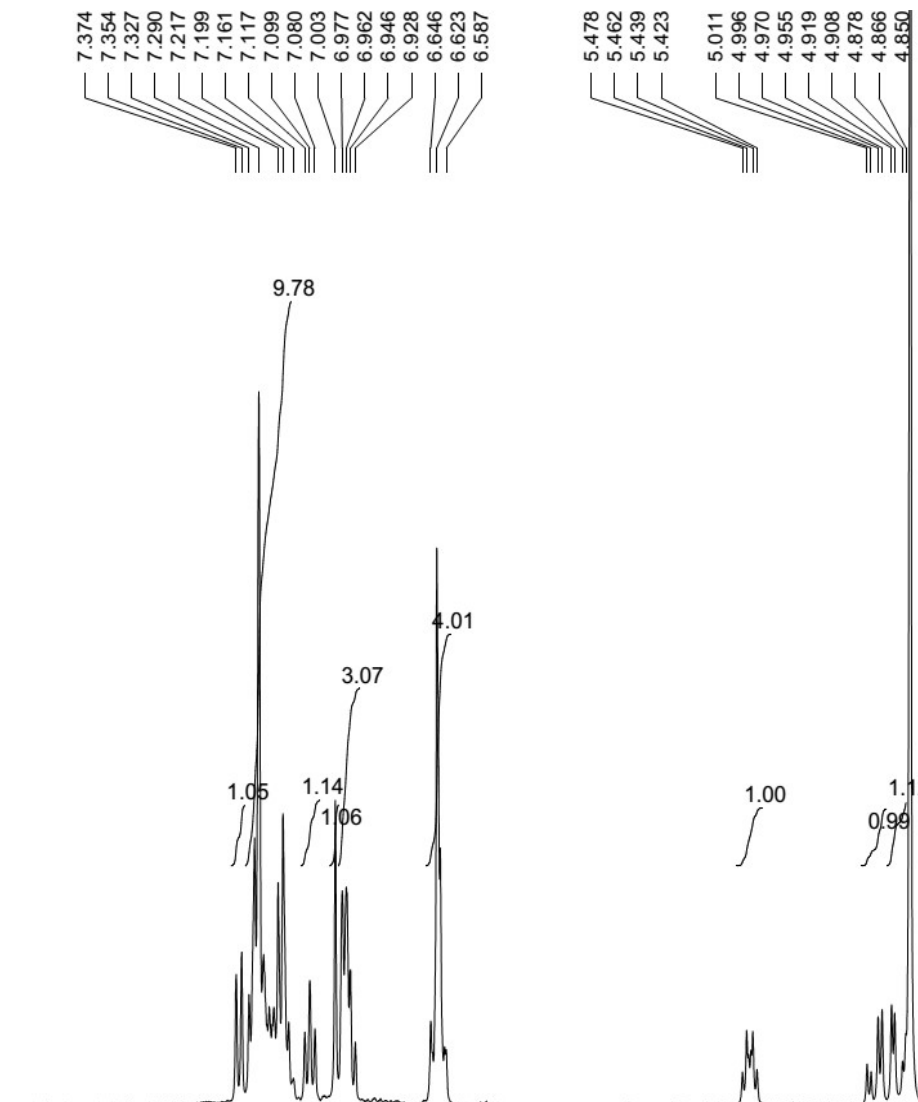

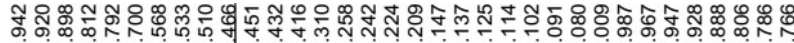

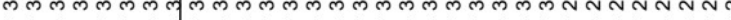

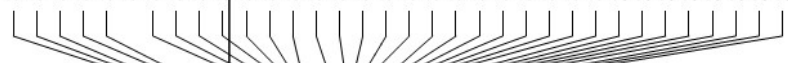

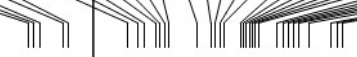<smiles>COc1ccc(Cn2c(CCc3ccccc3)nnc2CNC(=O)CNC(=O)C(C)Cc2ccccc2)cc1</smiles>

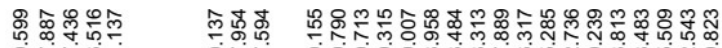

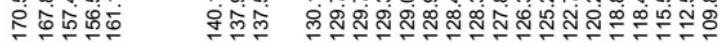
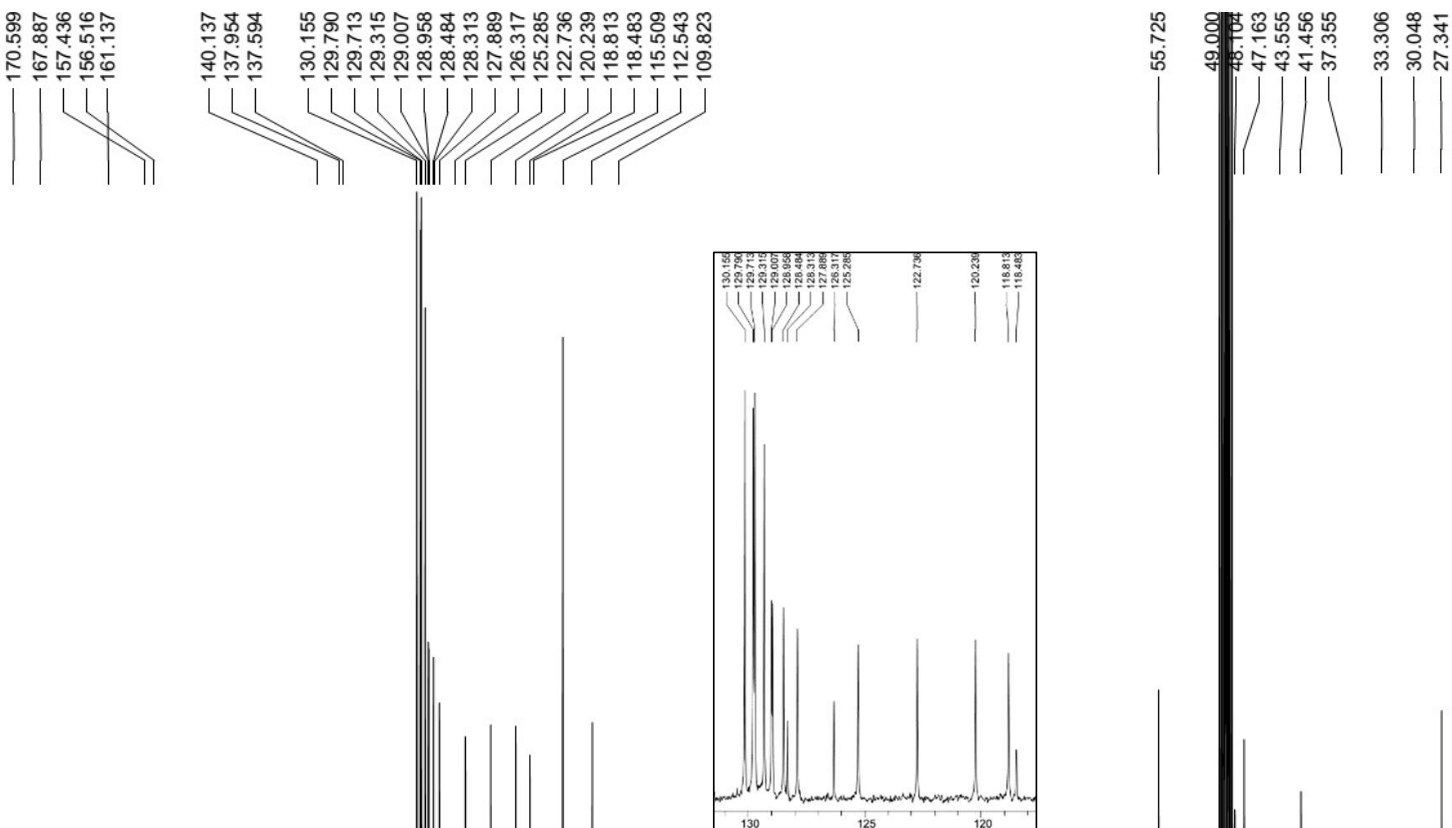

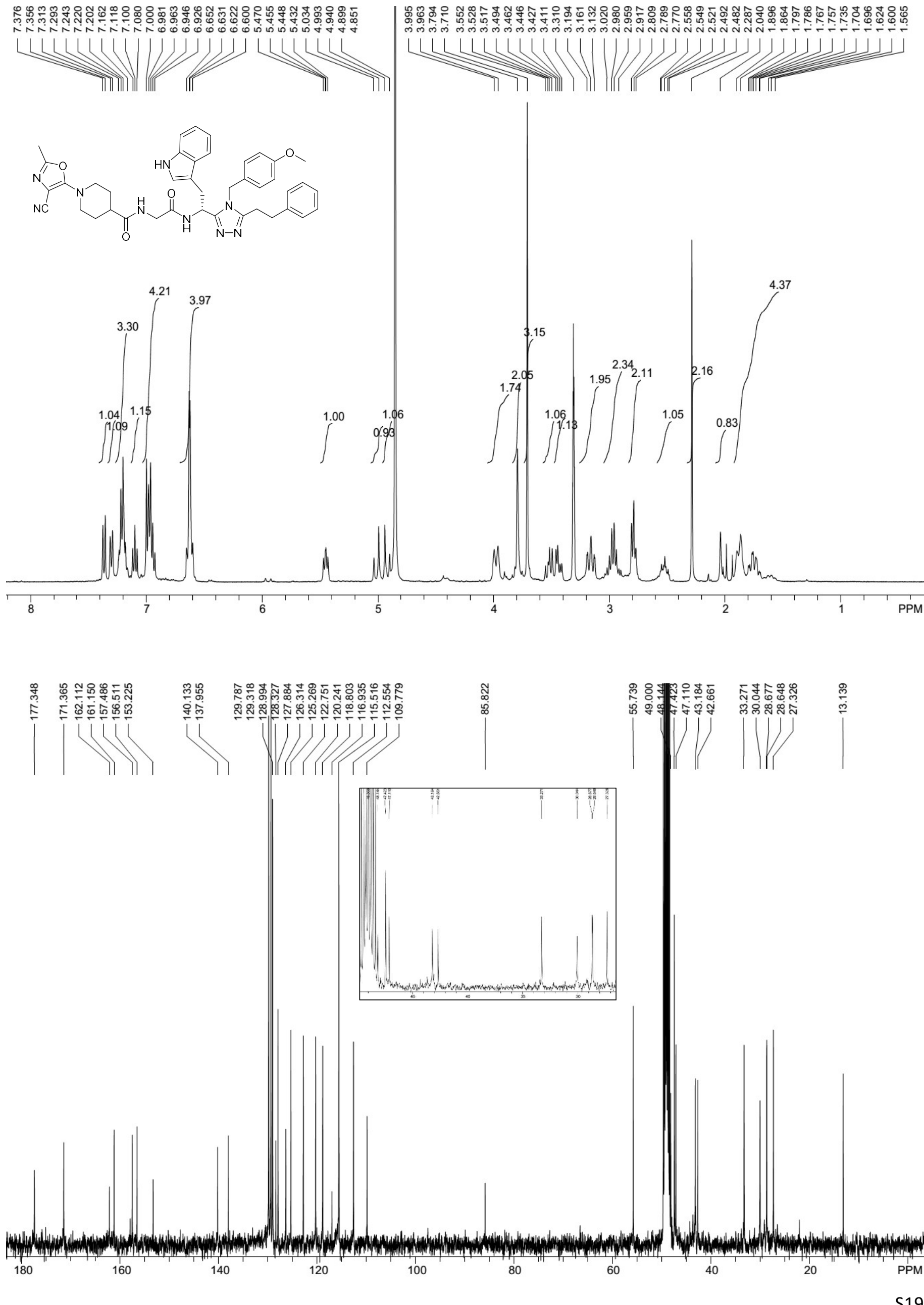

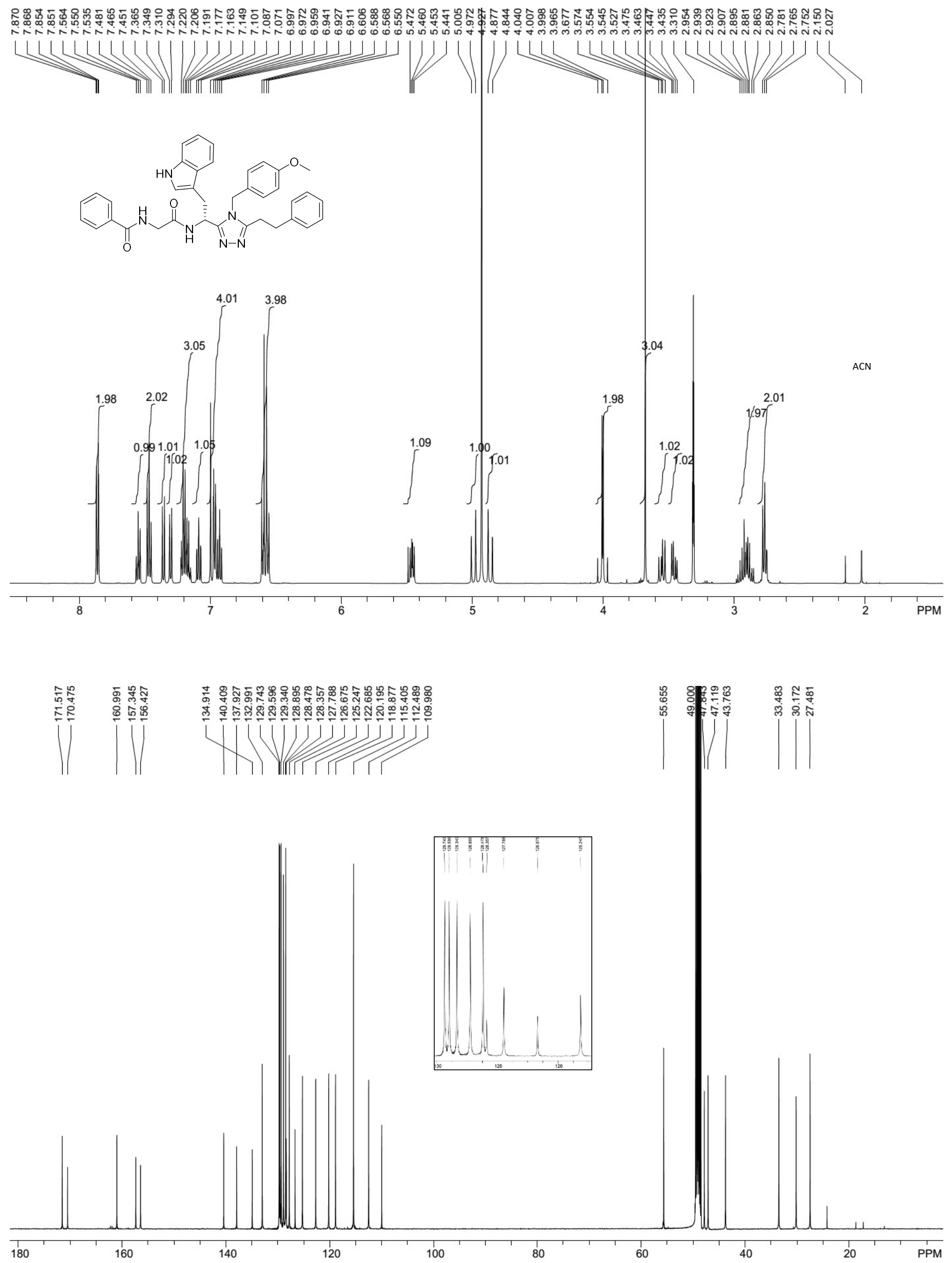
Compound 20

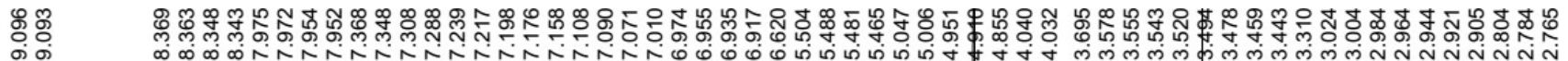

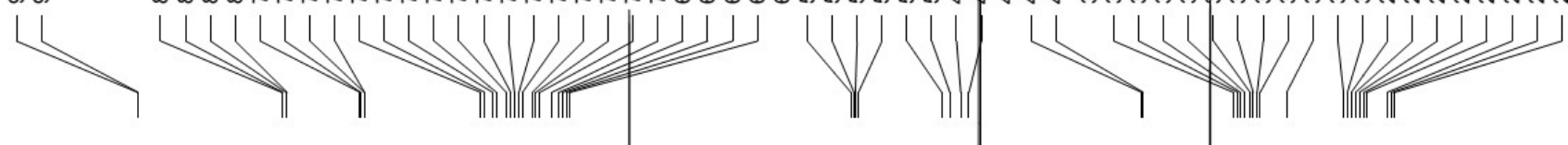<smiles>COc1ccc(CCc2nnc(CCc3ccccc3)n2Cc2c[nH]c3ccccc23)cc1</smiles>

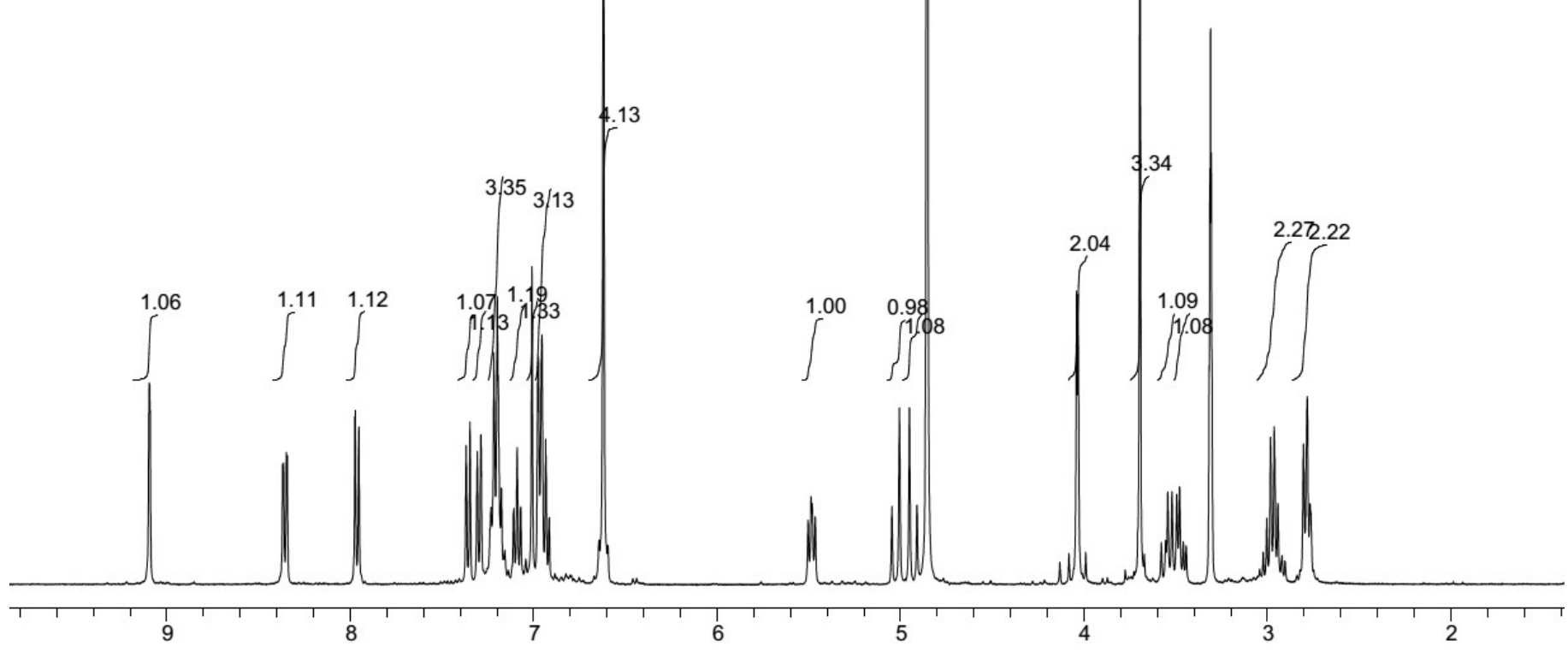

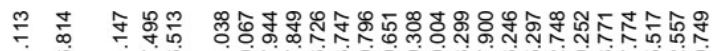

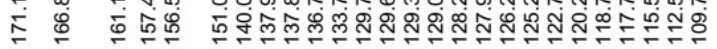
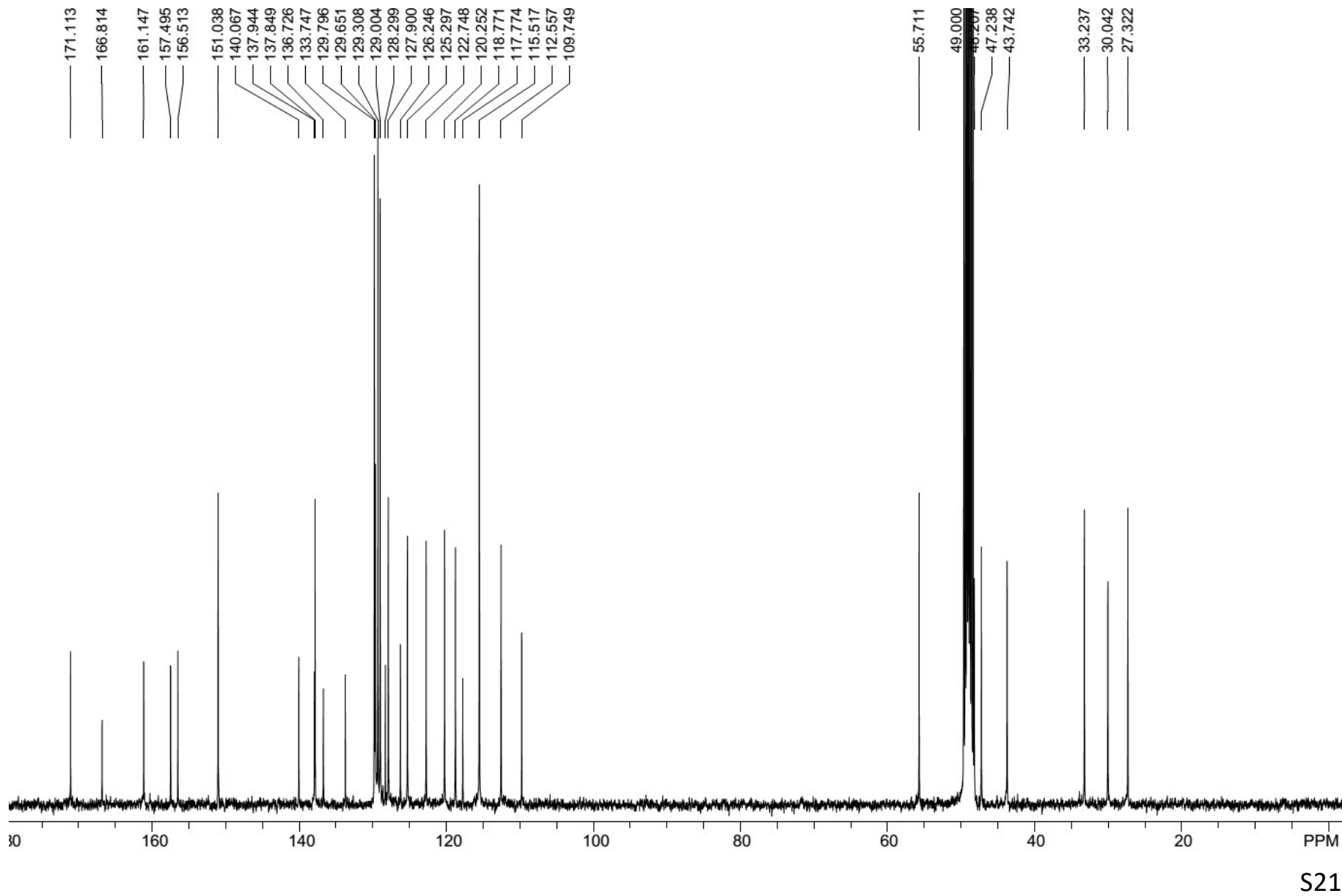


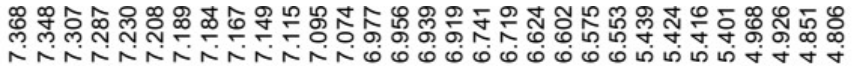
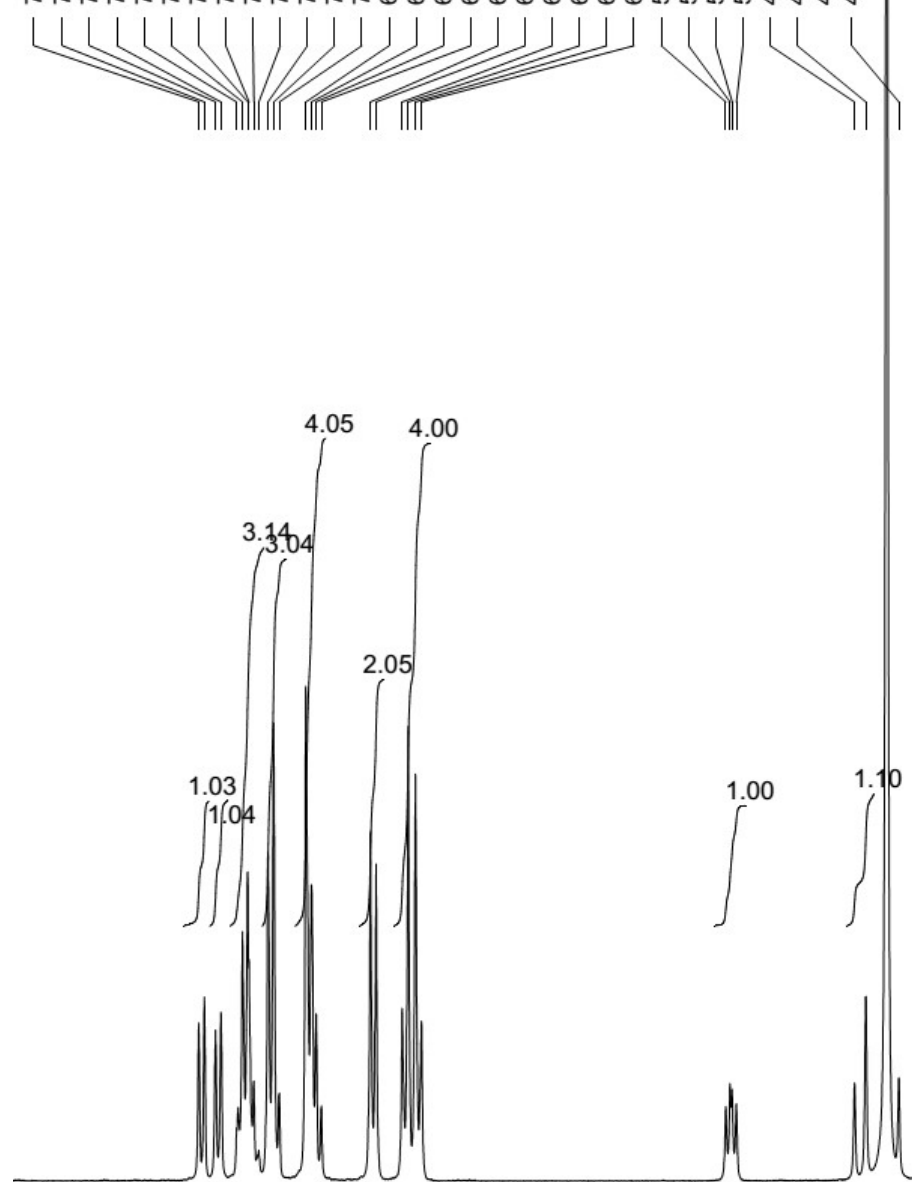

8
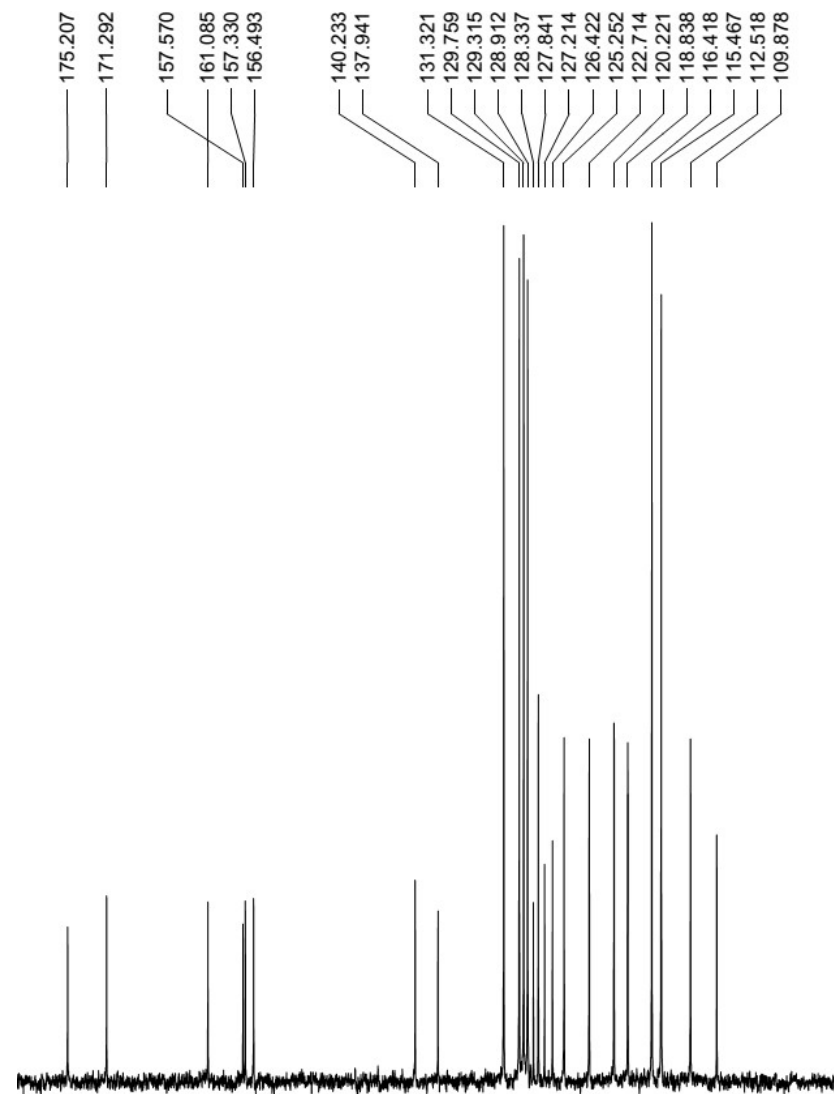

80 160 120

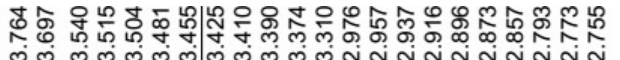
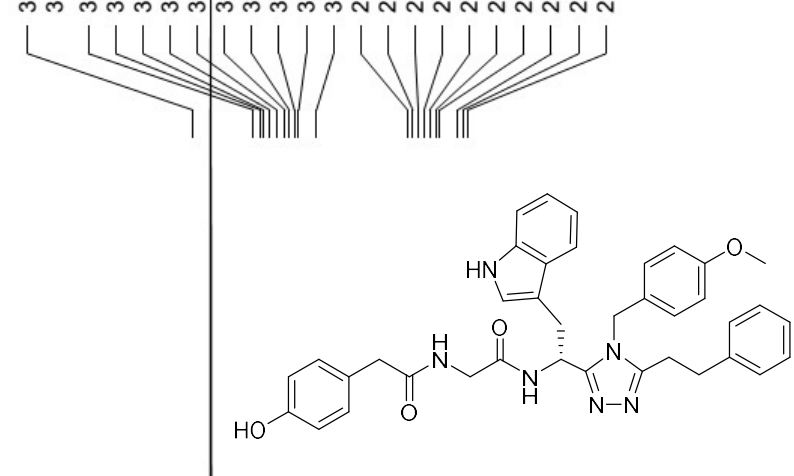

04
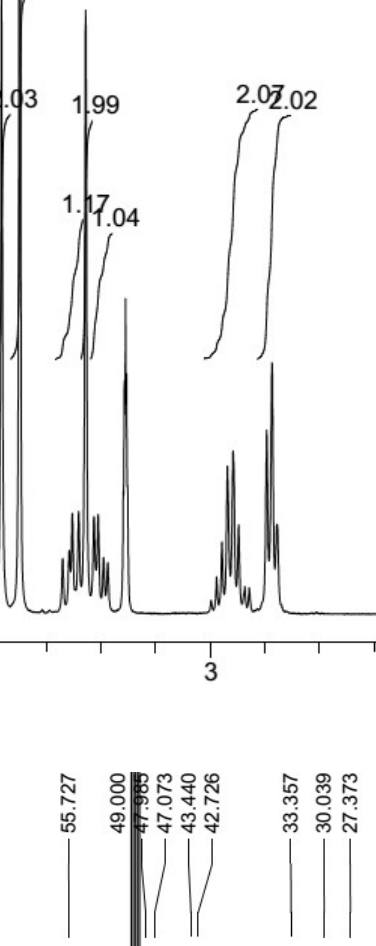


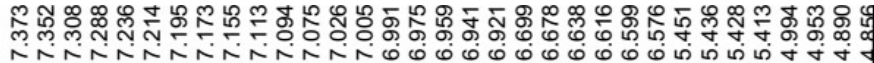

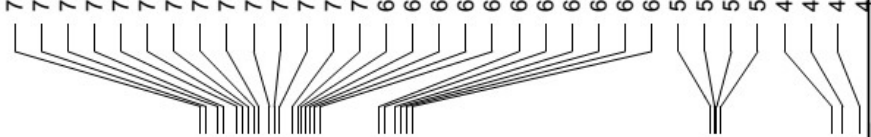<smiles>COc1ccc(CCc2nnc(NC(=O)CC(=O)NC(=O)CCc3ccccc3)n2Cc2ccccc2)cc1</smiles>

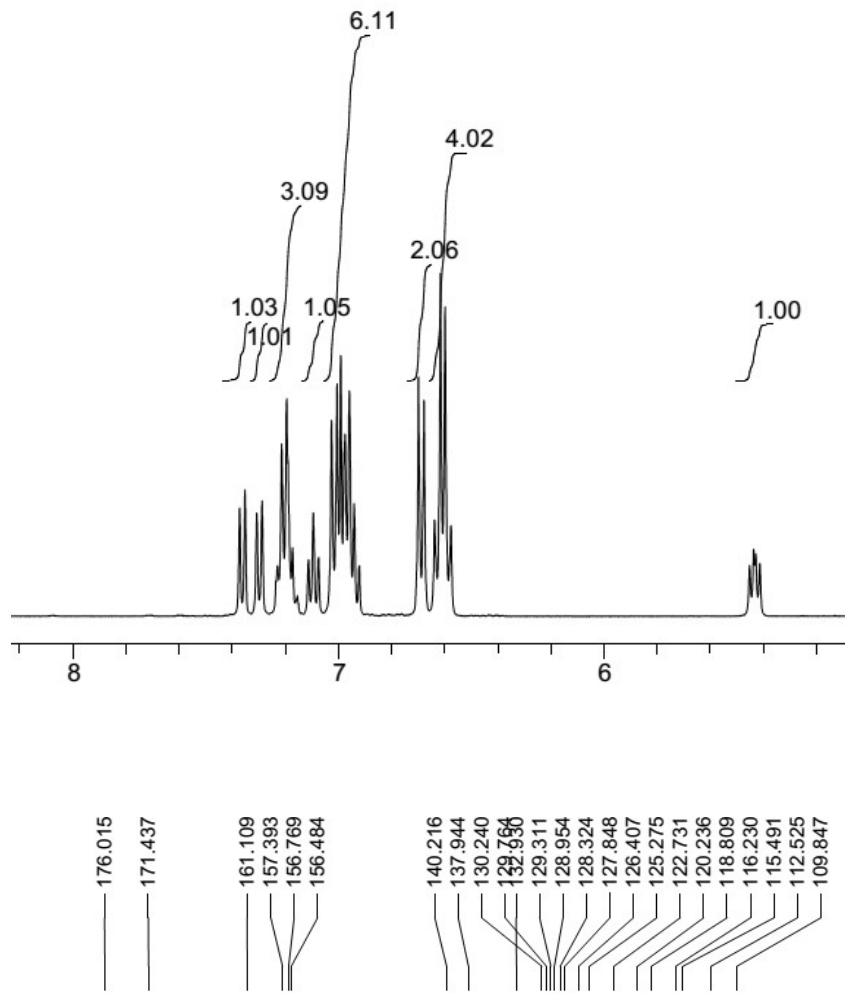

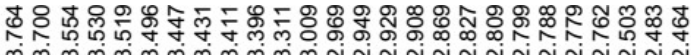
D.

ๆ $|\|\mid\| \|$
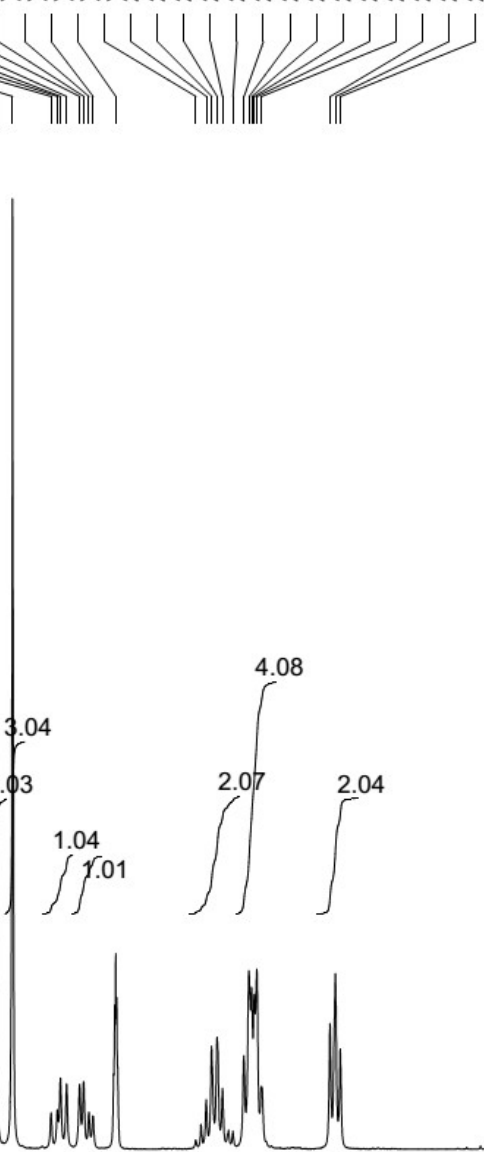

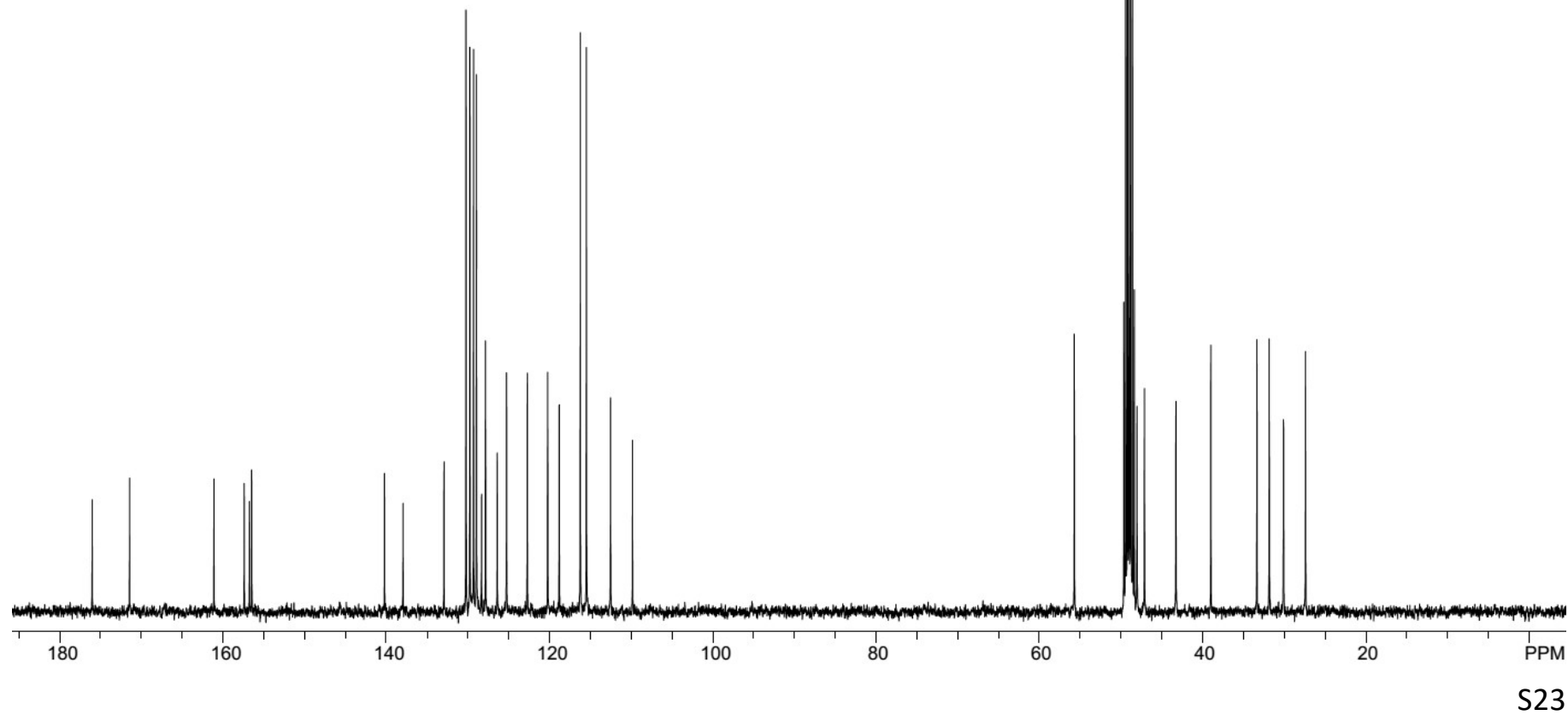




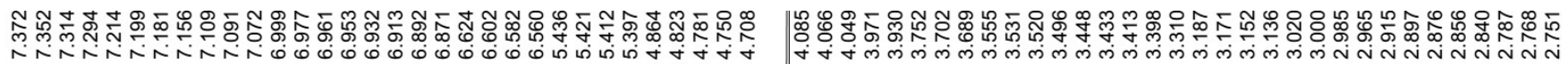
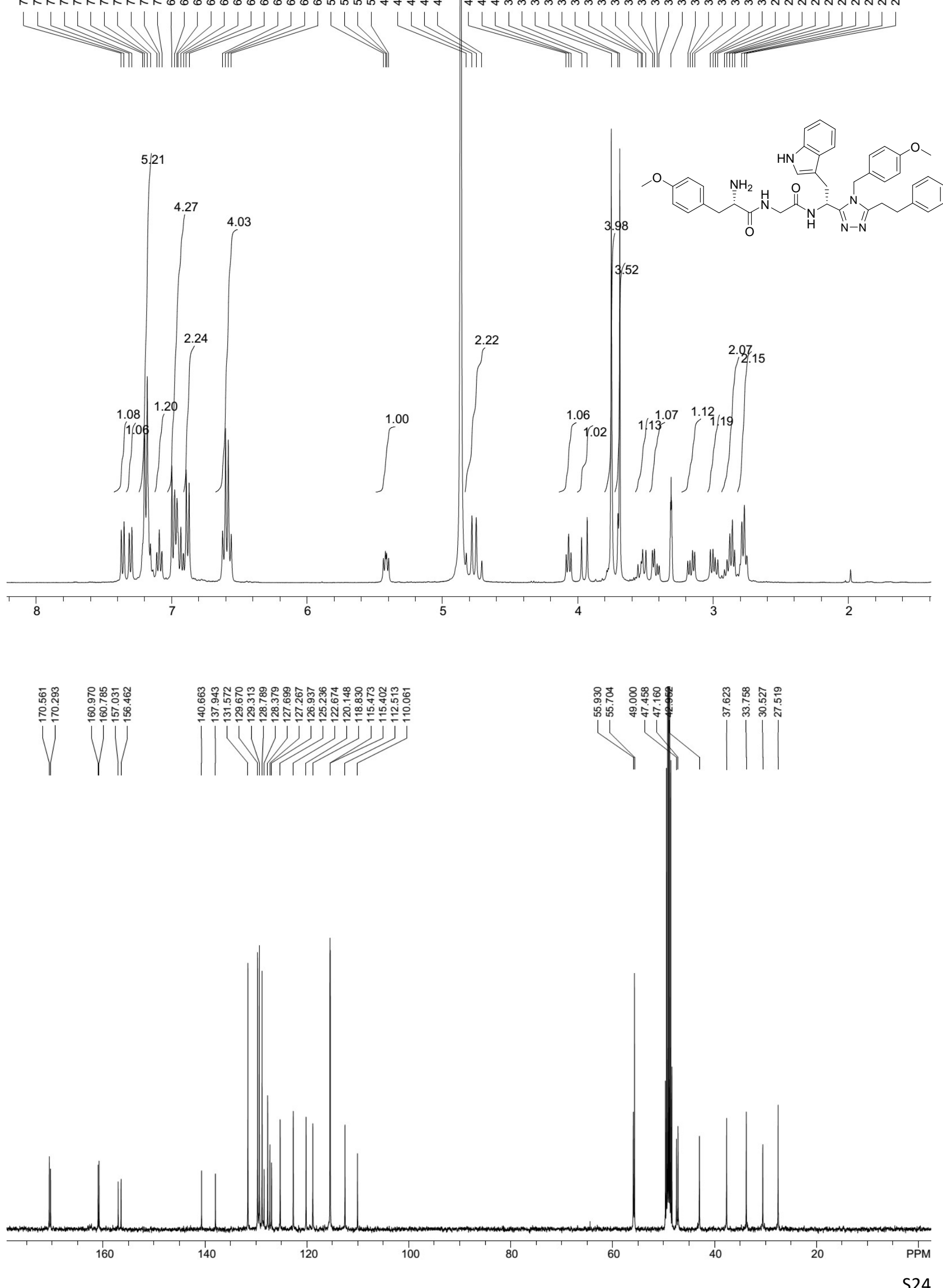


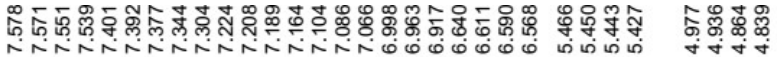

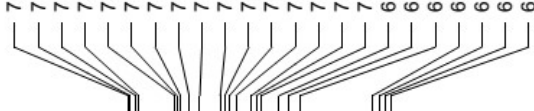

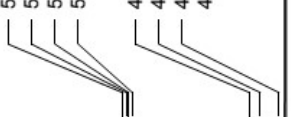<smiles>COc1ccc(Cn2c(CCc3ccccc3)nnc2C(Cc2ccccc2)NC(=O)CC(=O)N/C=C/c2ccccc2)cc1</smiles>

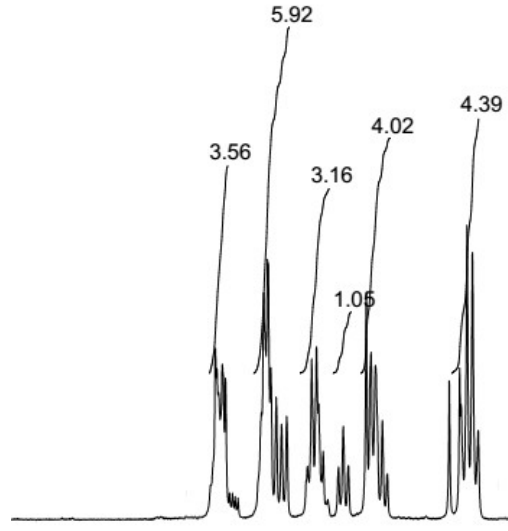

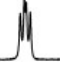

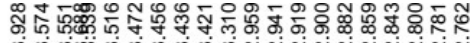

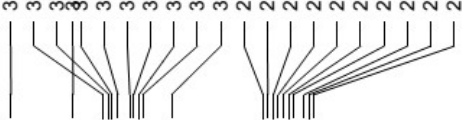

8
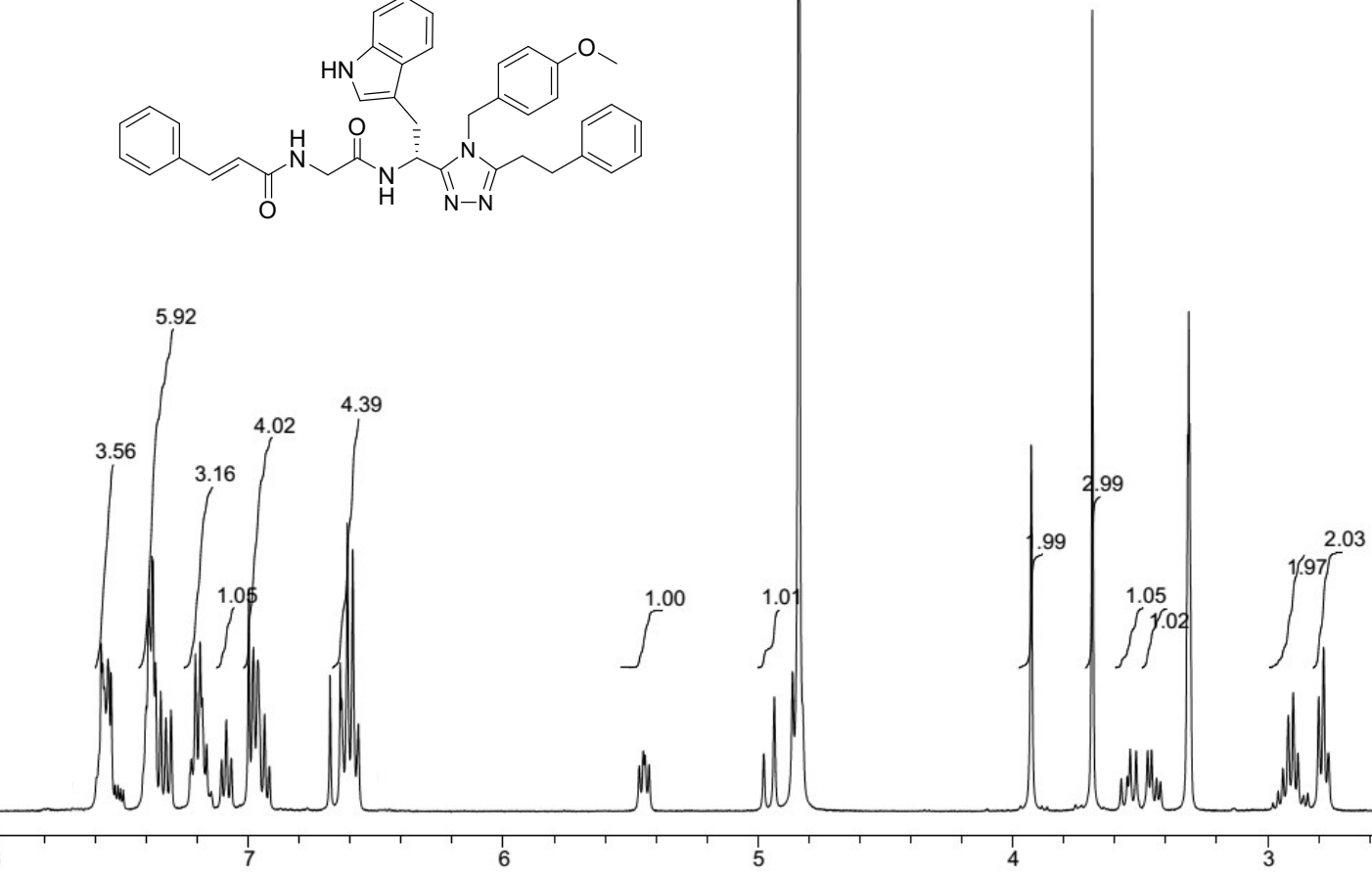

6
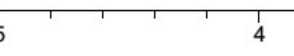

3

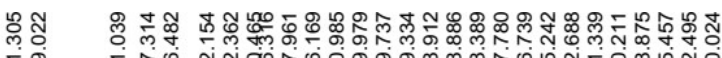

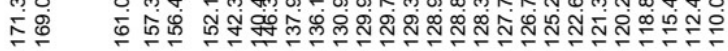

† T)
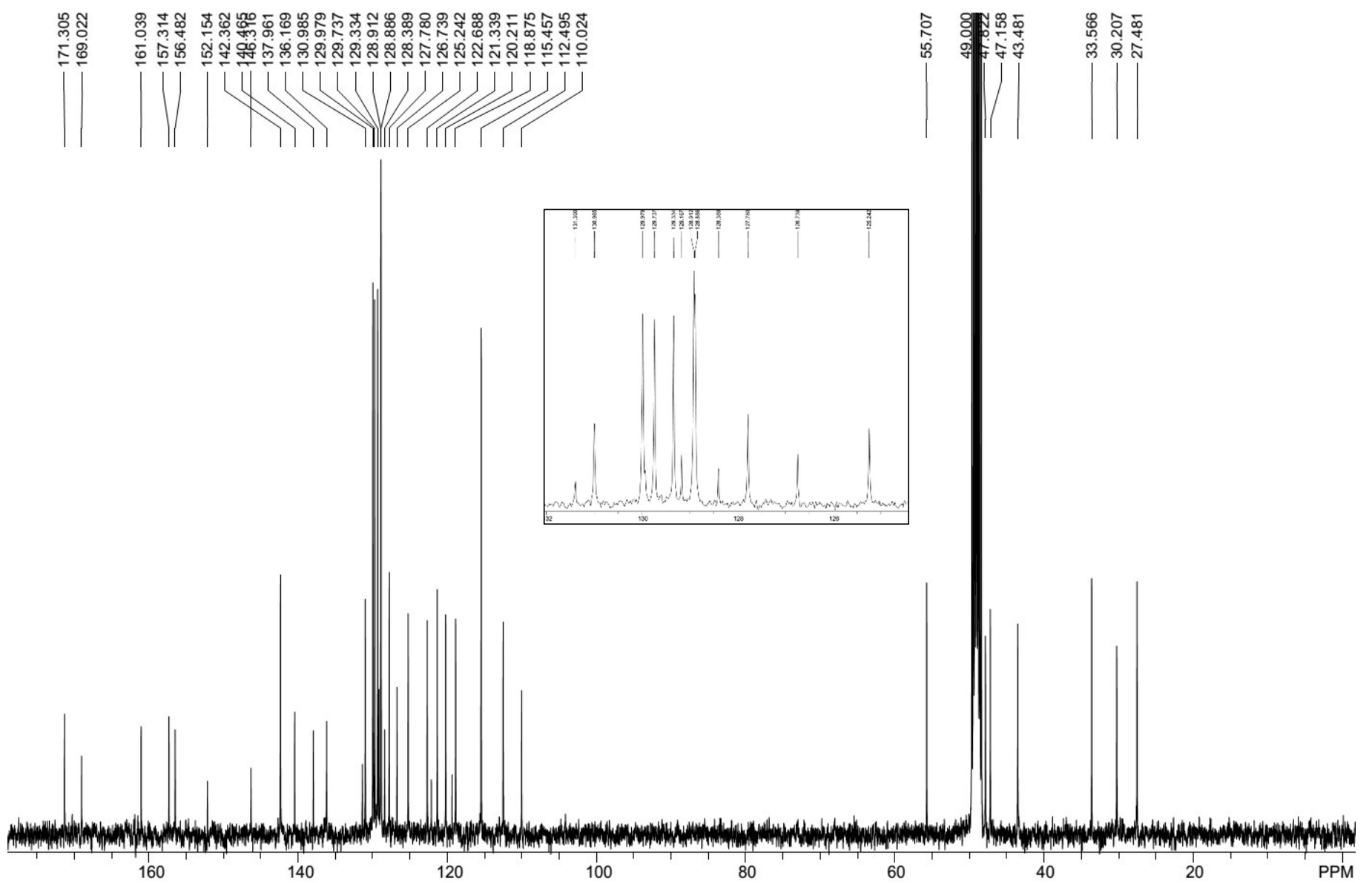


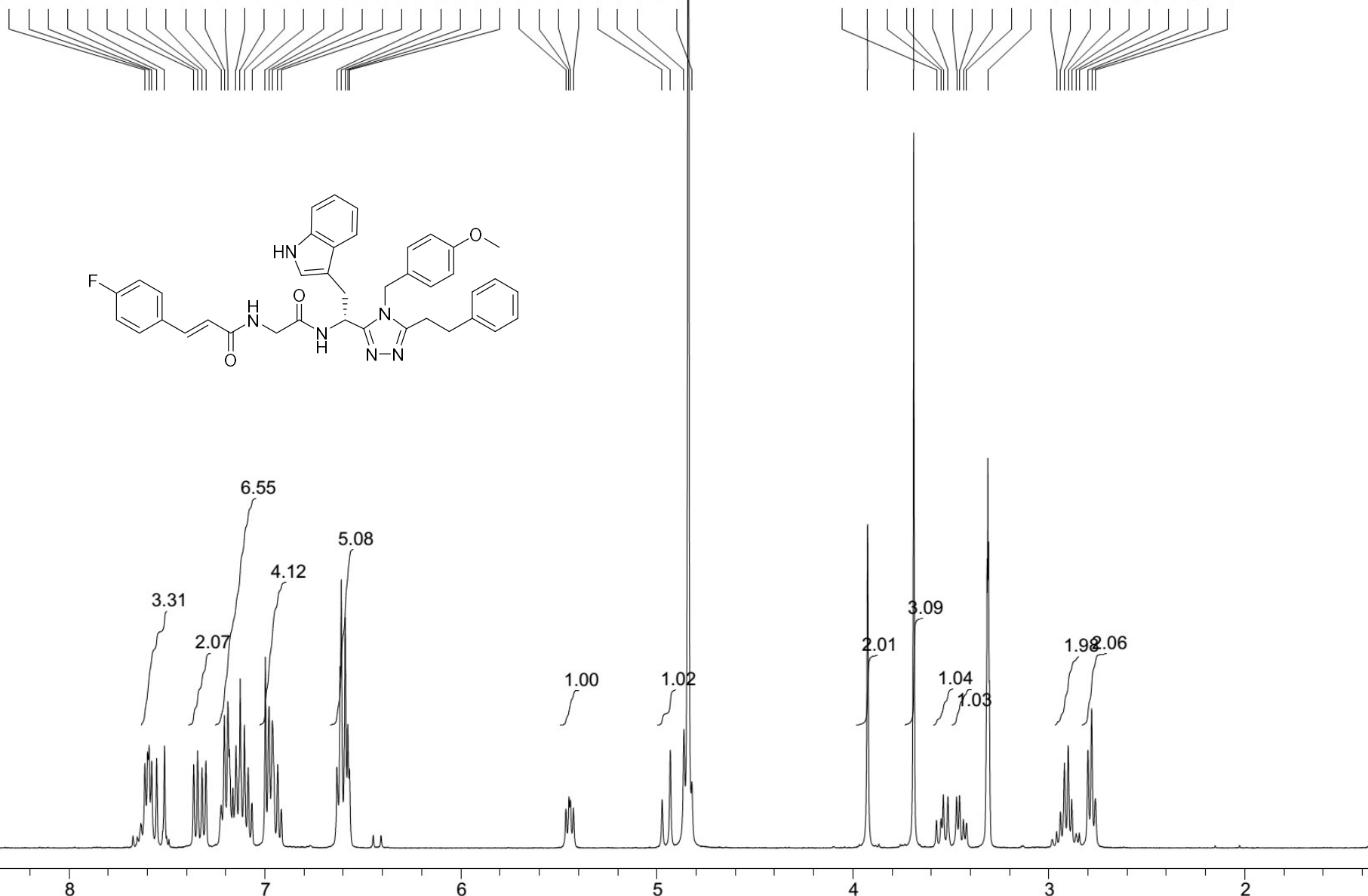

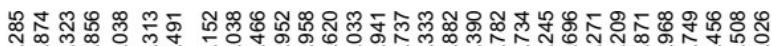

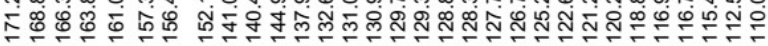
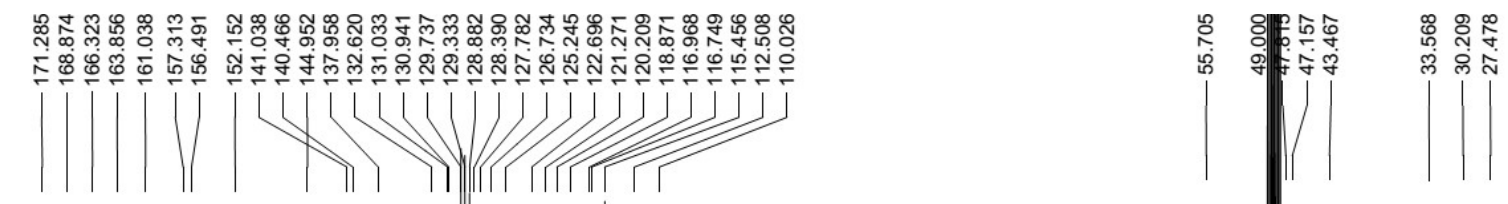


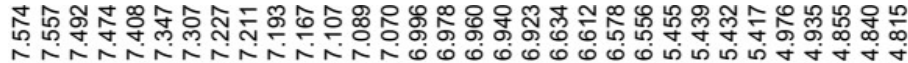
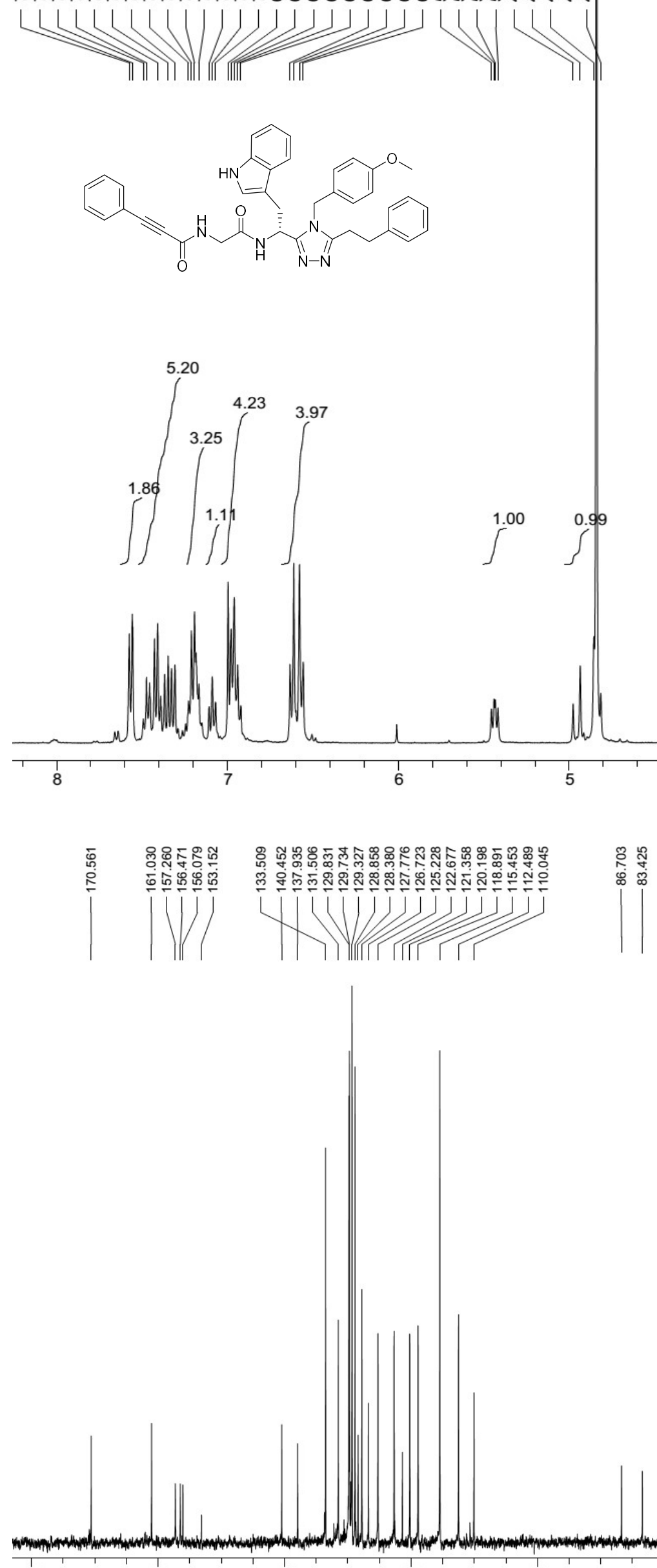

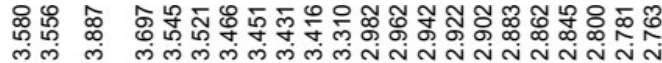

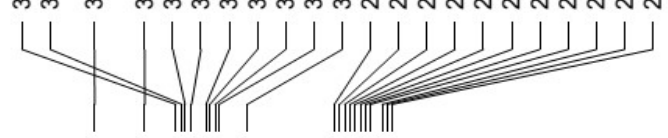




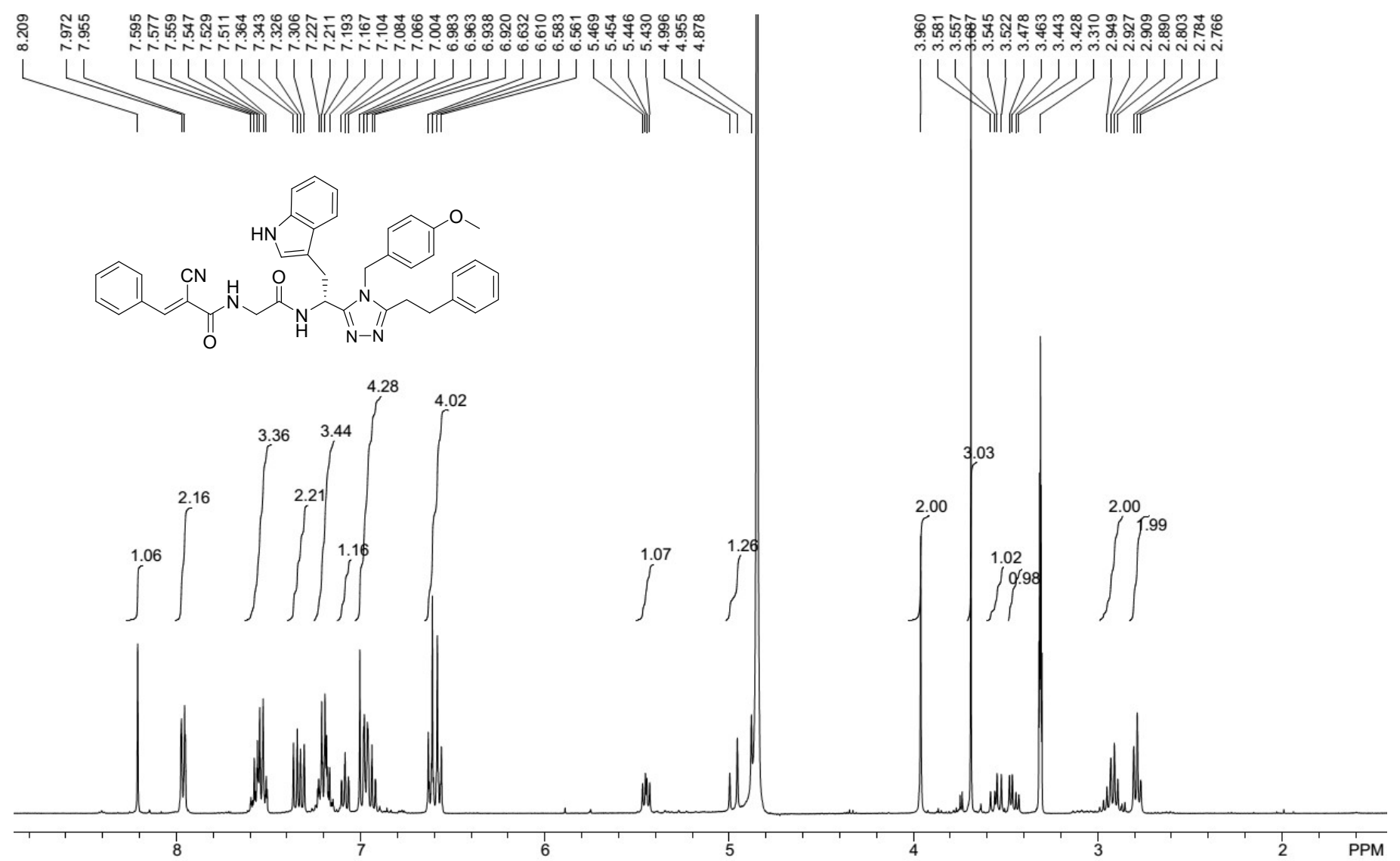

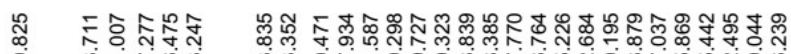

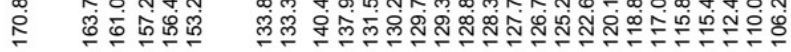
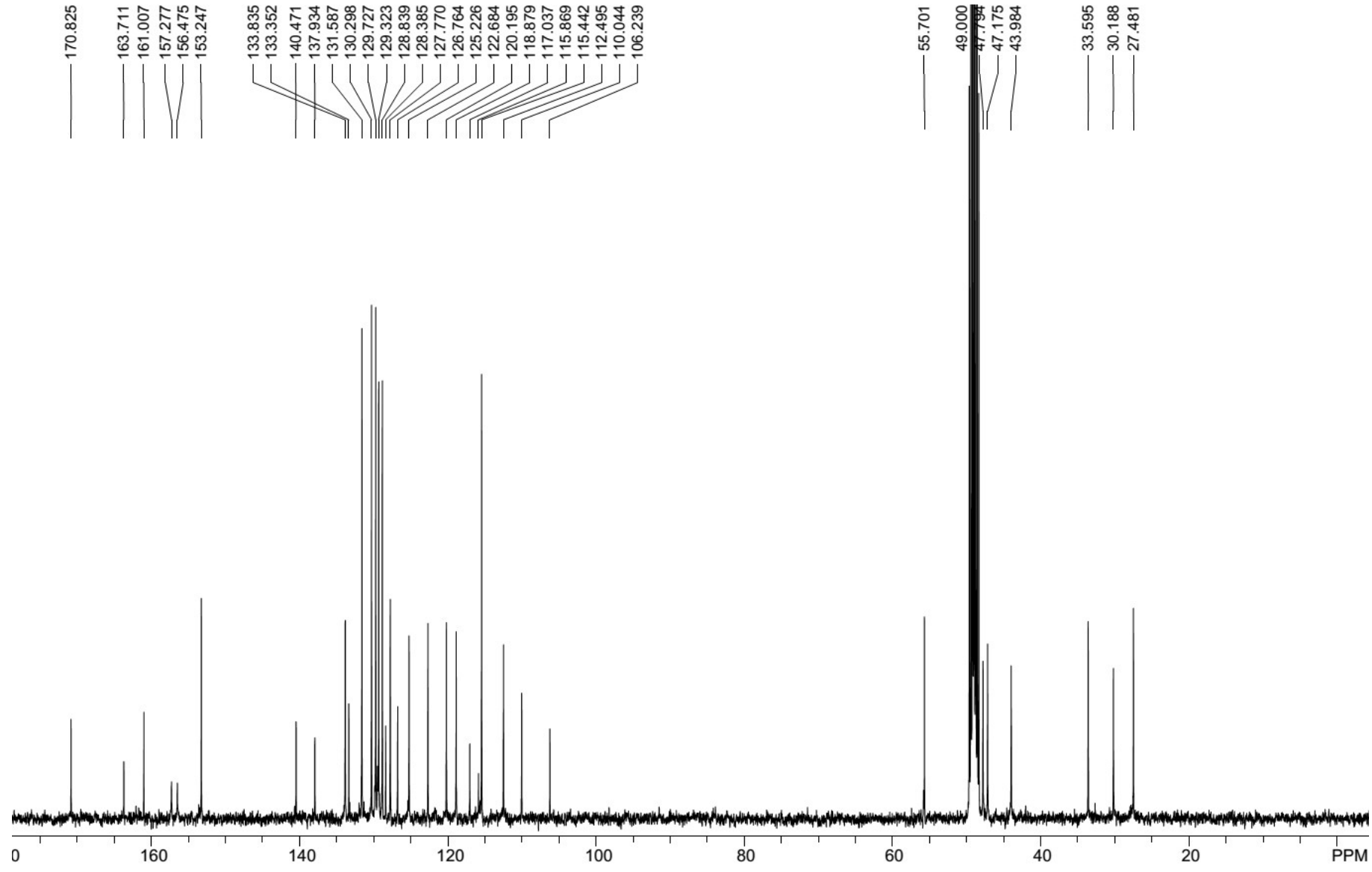


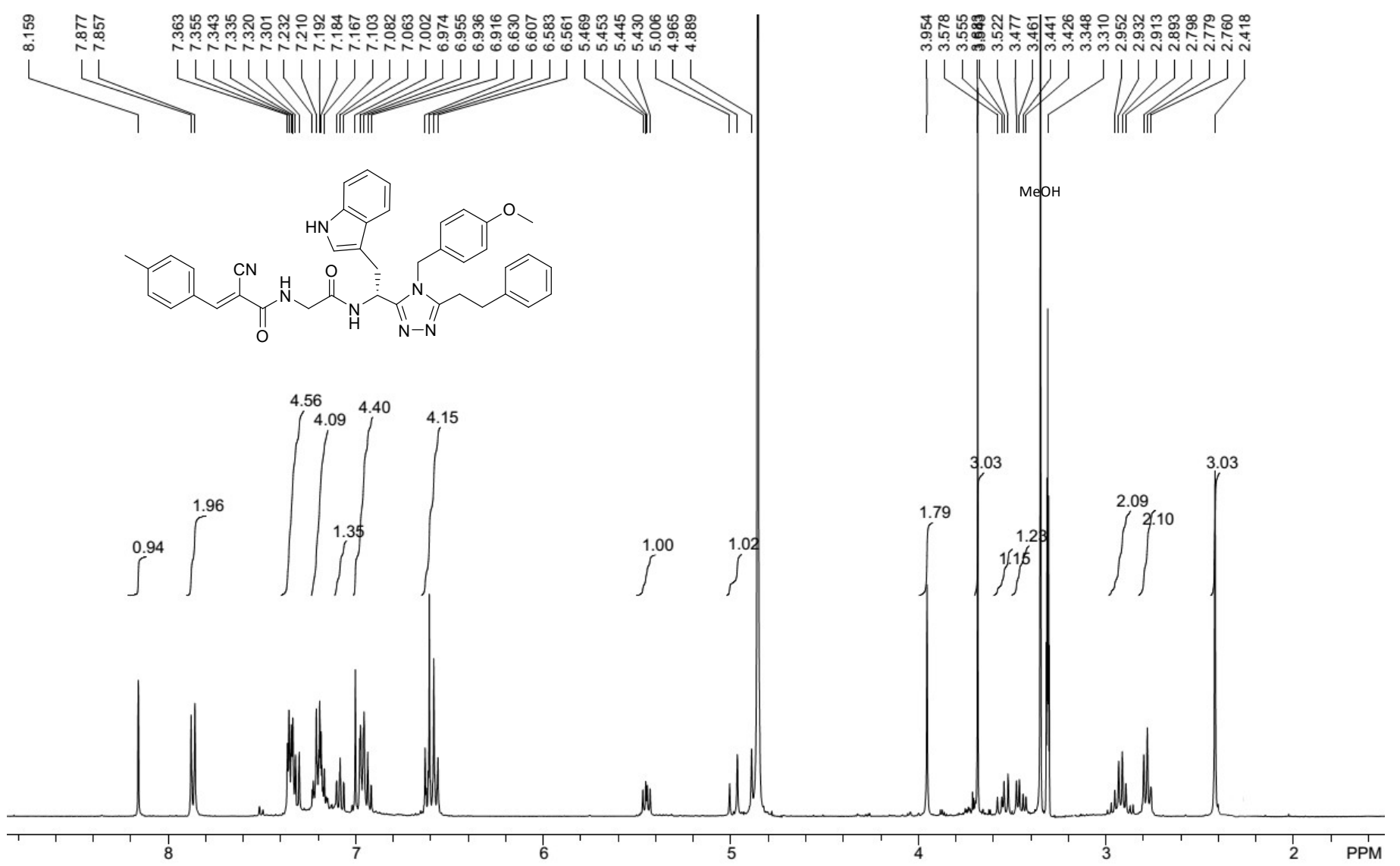

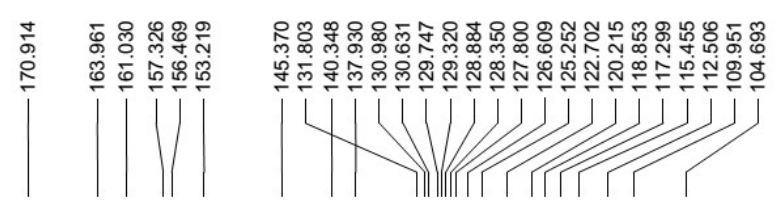

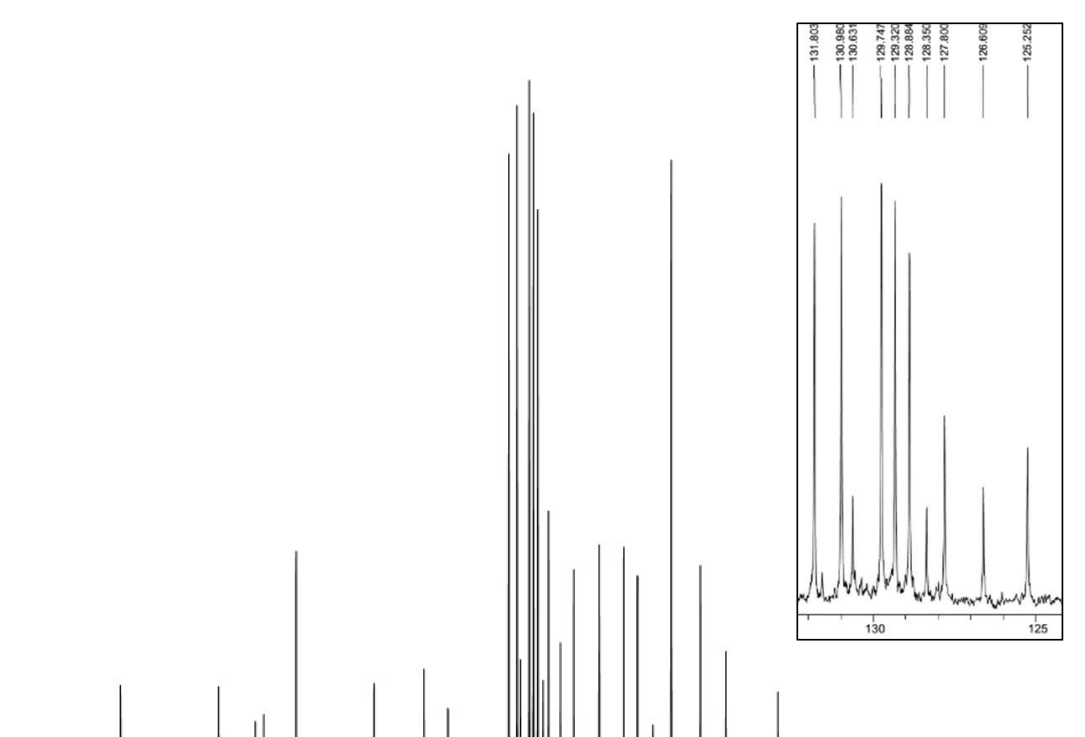

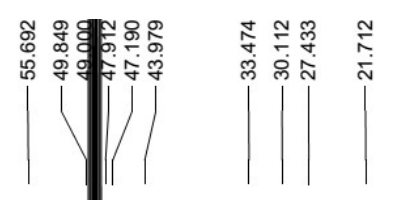

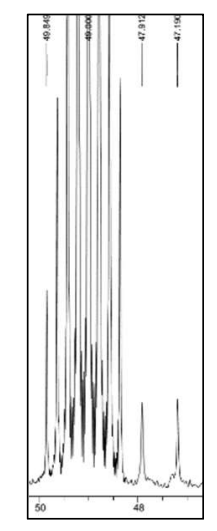




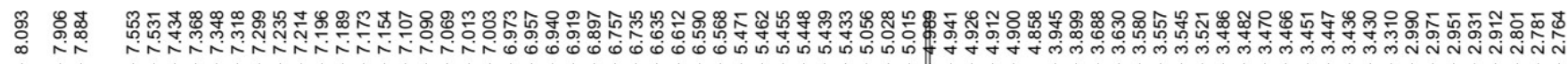

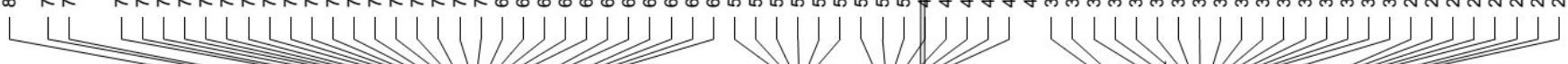<smiles>COc1ccc(Cn2c(CCc3ccccc3)nnc2CC(=O)NCC(=O)NCC(=O)C(C#N)(Cc2ccccc2)Cc2ccc(O)cc2)cc1</smiles>

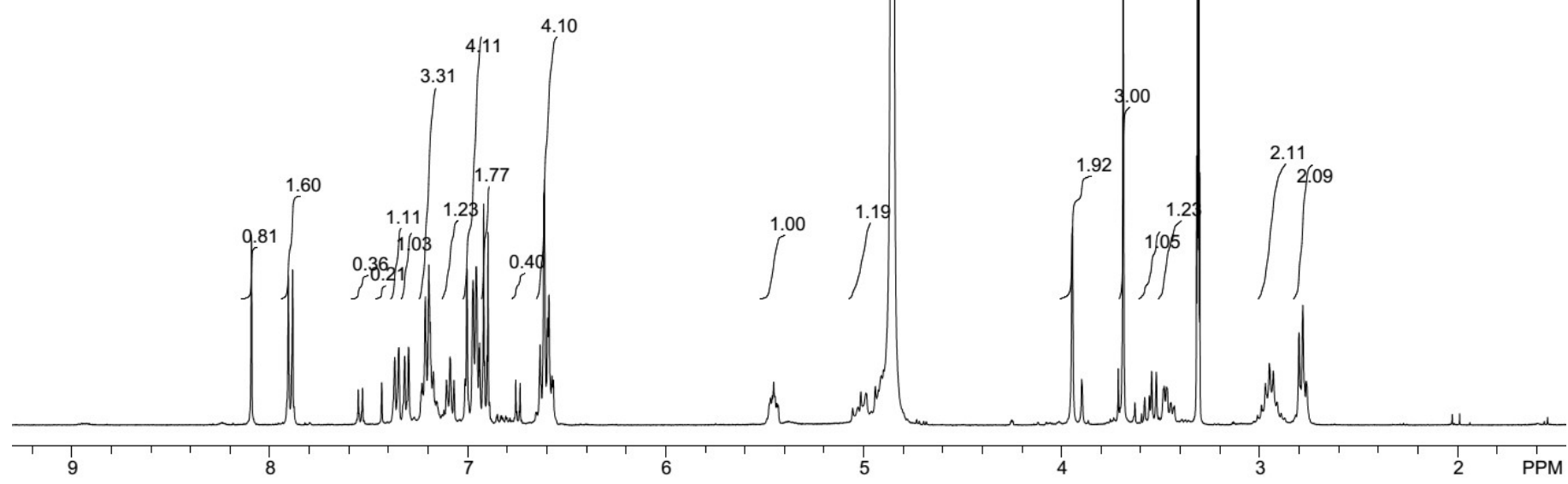

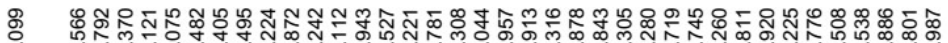

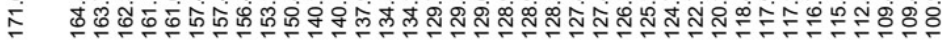

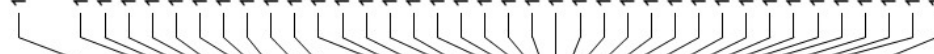

\ गो।
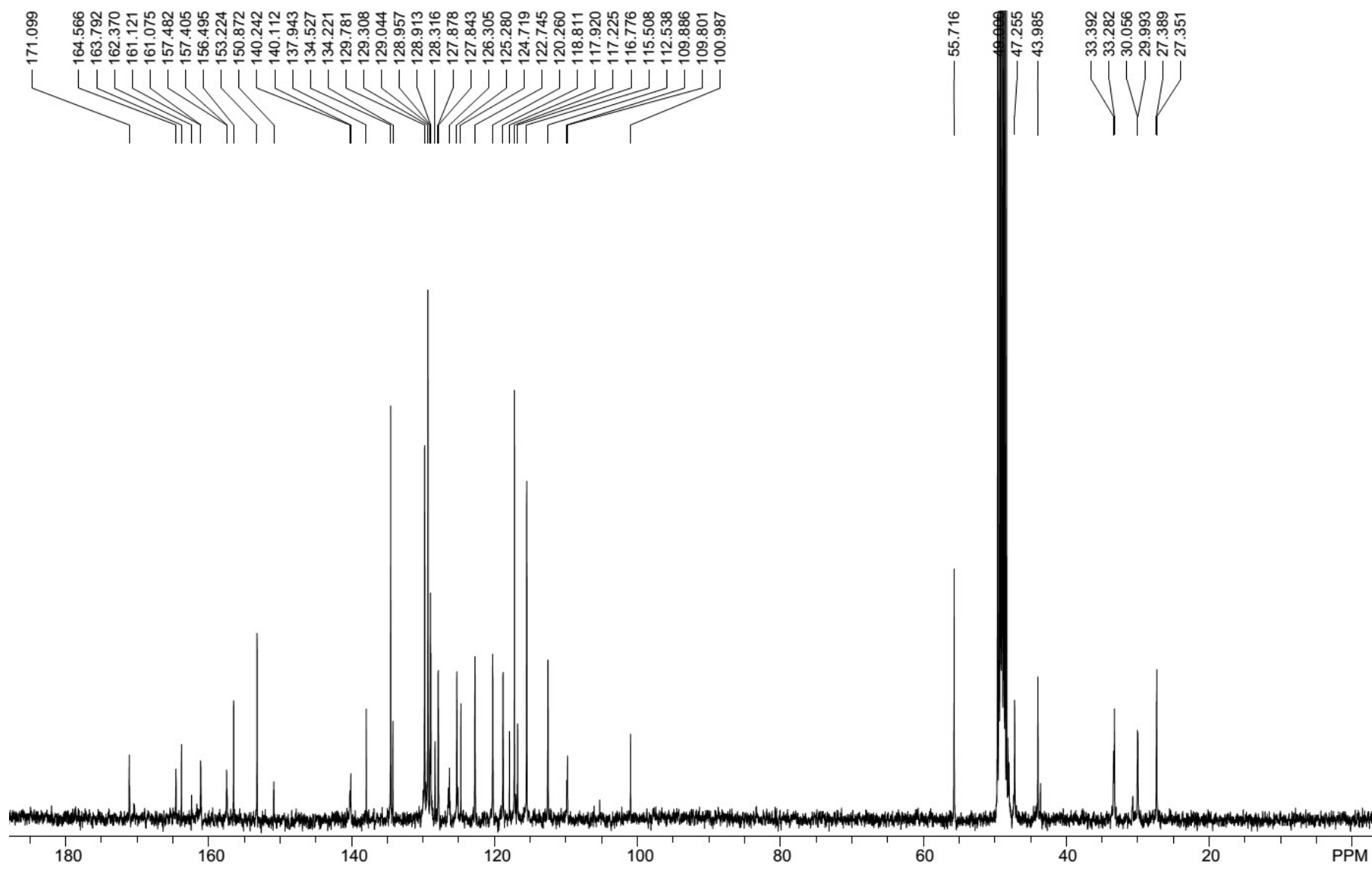

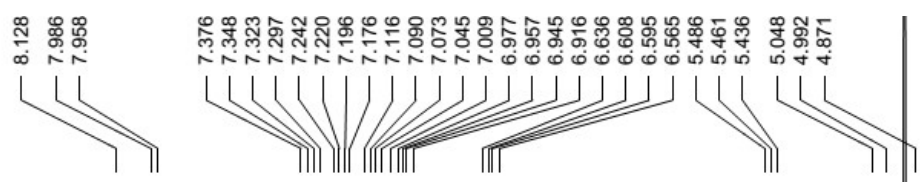

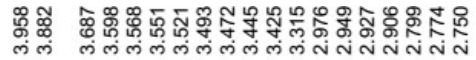

LL LULU)।

7 Y IIII NWIII
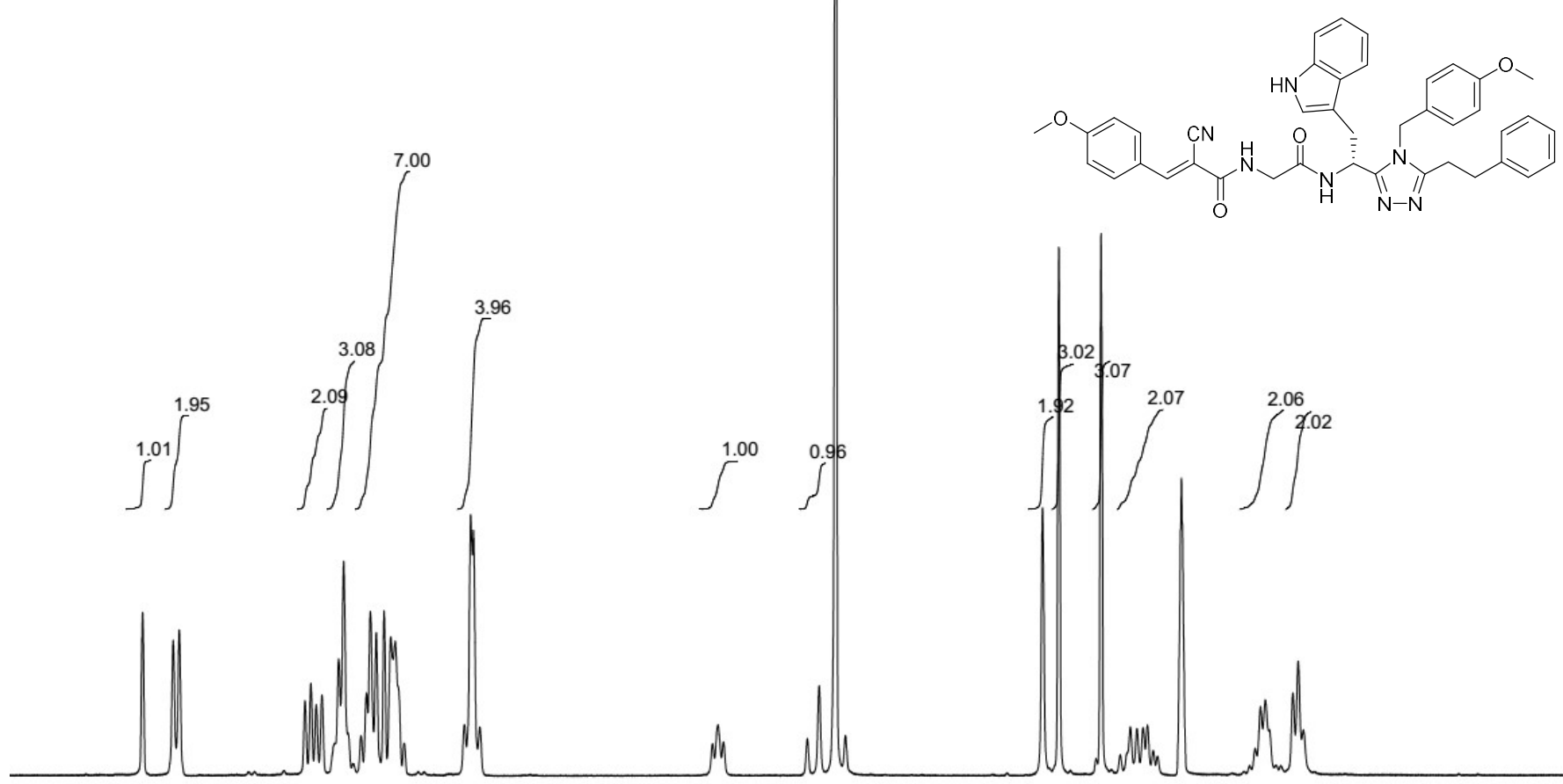

$\int^{2.06} 2.02$
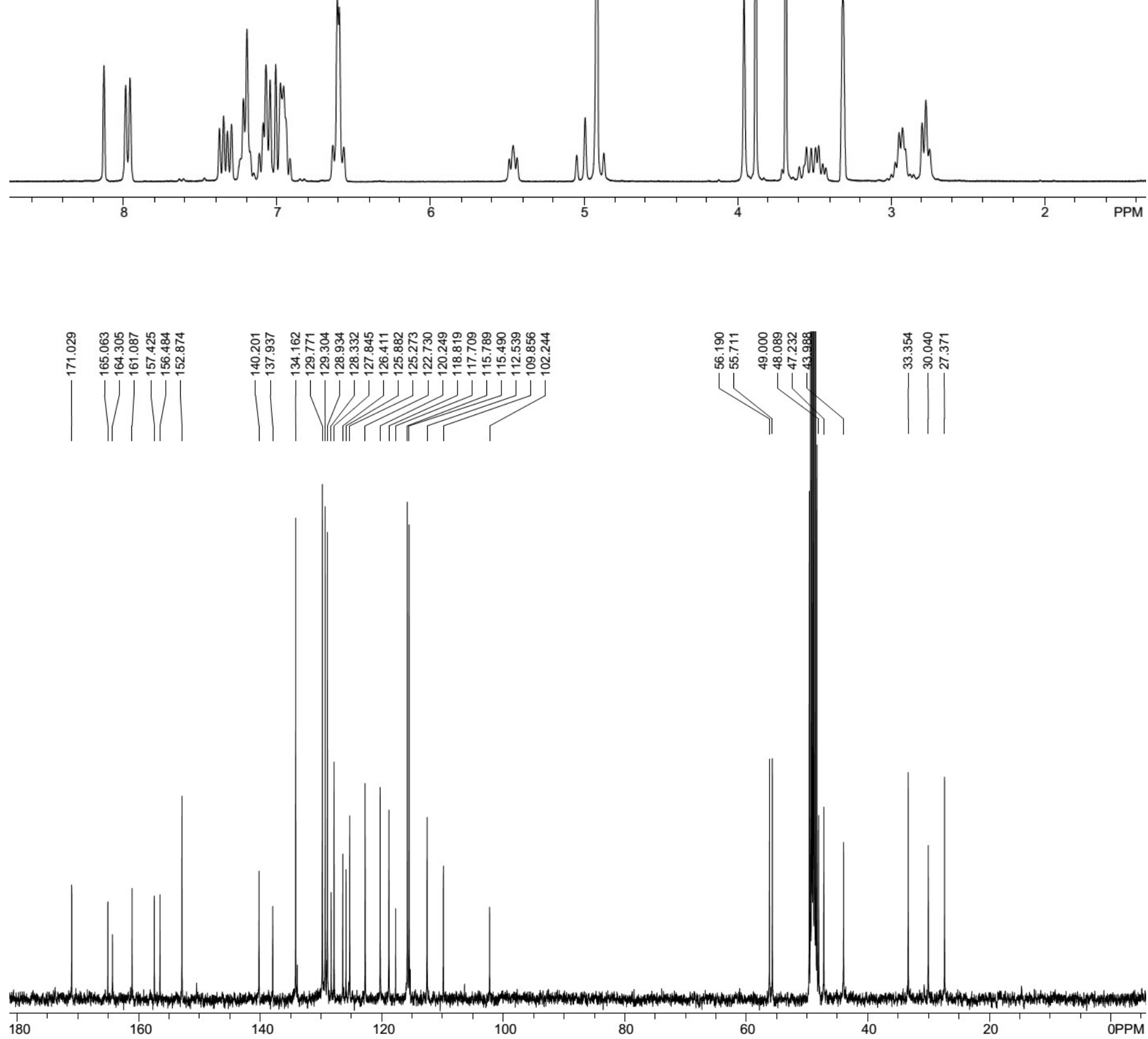

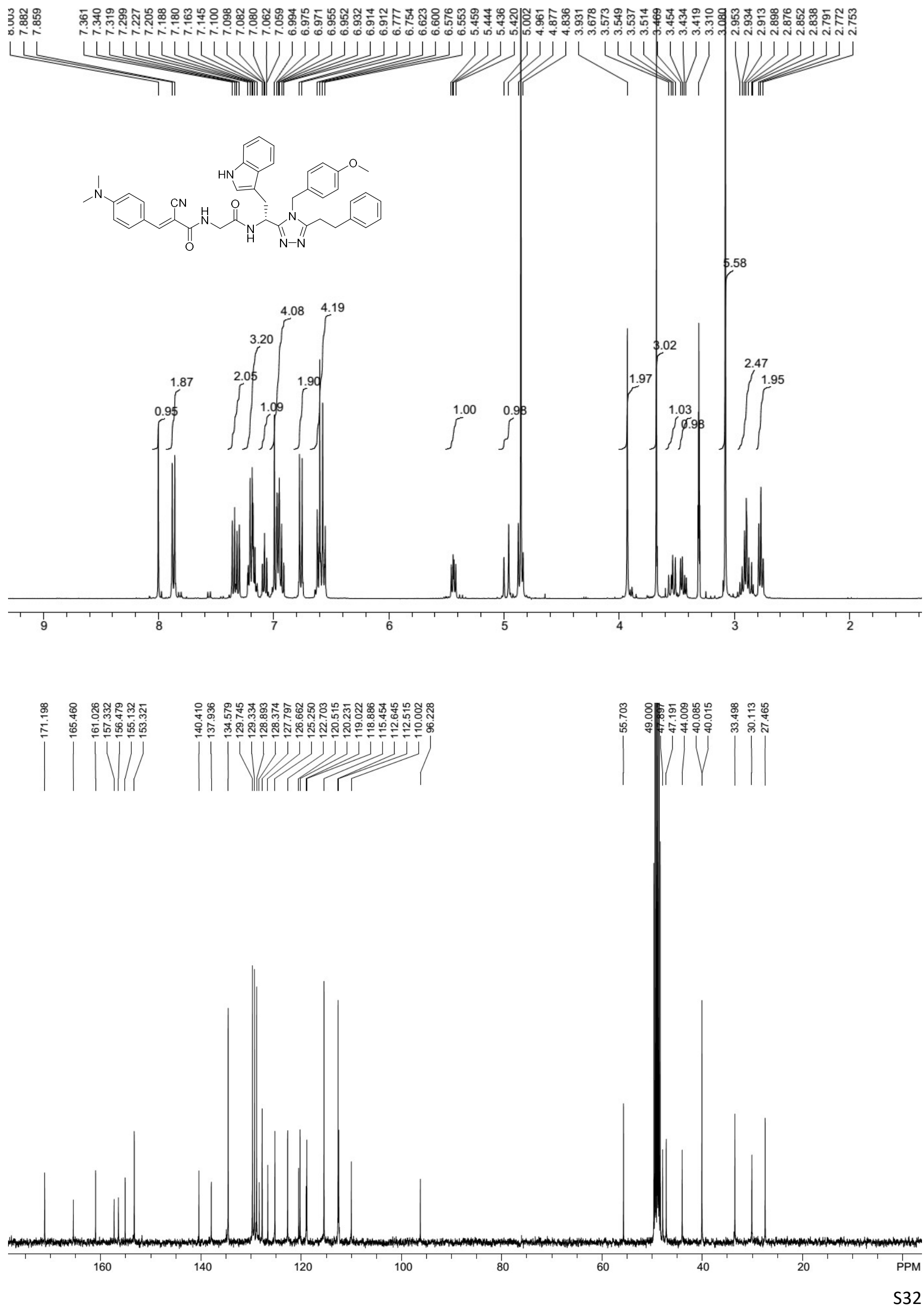


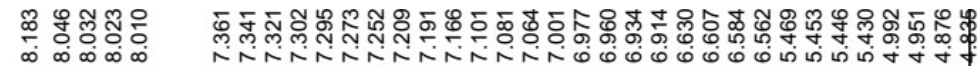

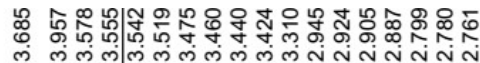

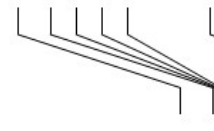

แMU M
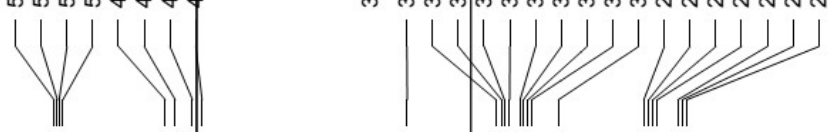<smiles>COc1ccc(CCc2nnn(CCc3ccccc3CCc3ccccc3)c2CC(=O)NCC(=O)NC(=O)/C(C)=C/c2ccc(F)cc2)cc1</smiles>
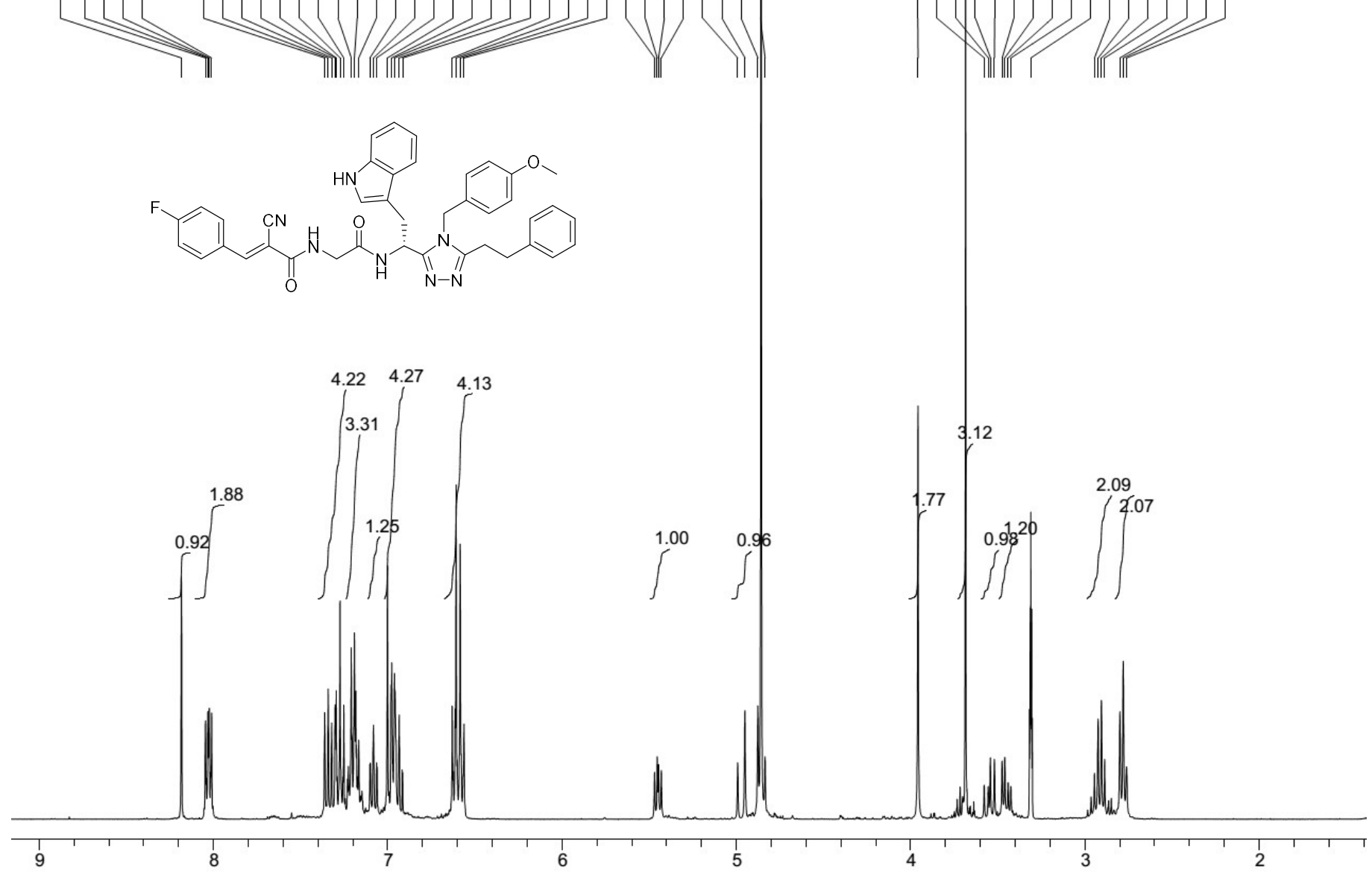

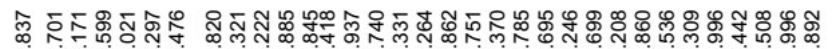

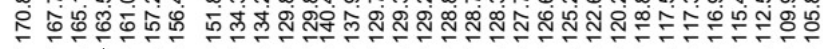
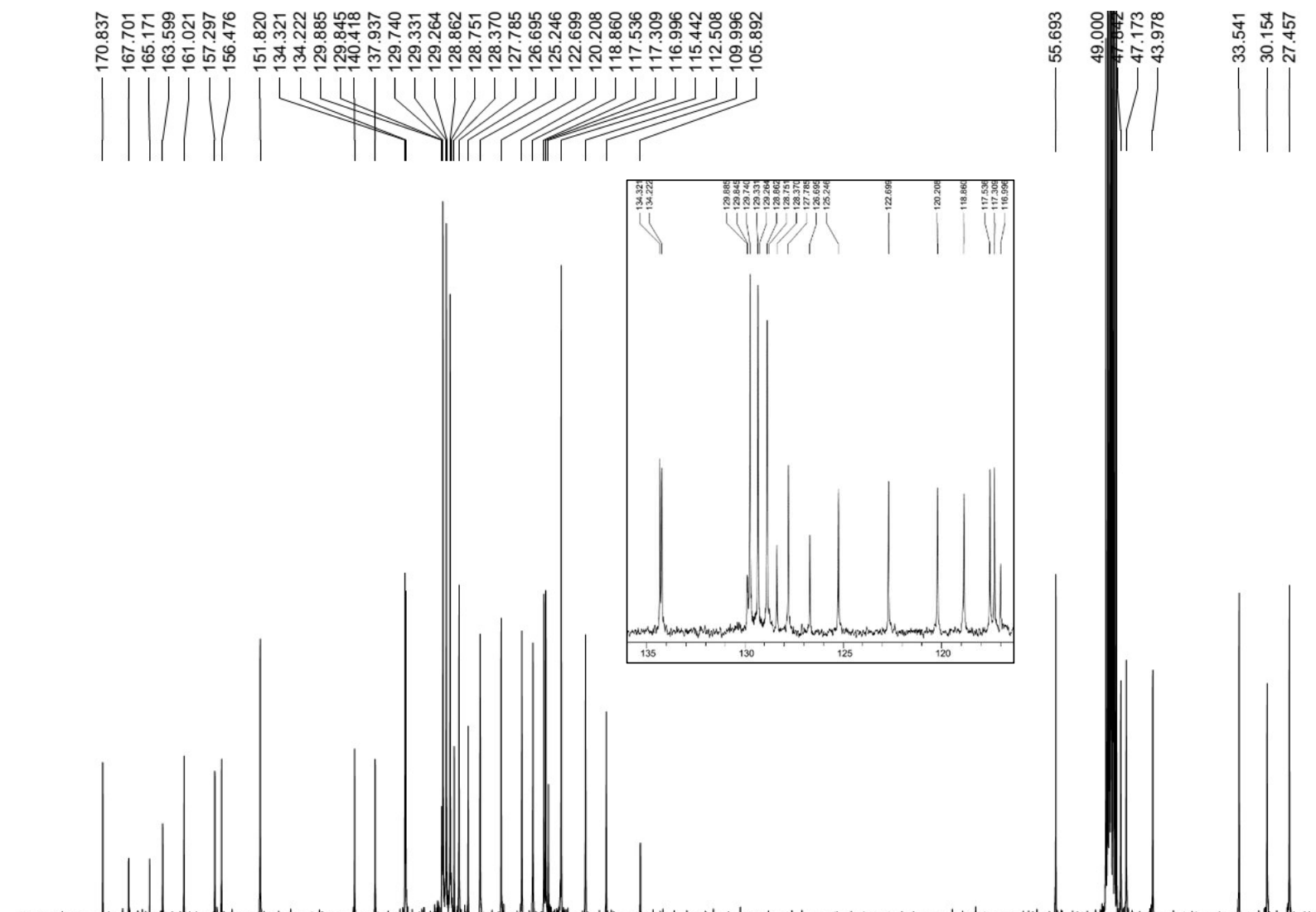


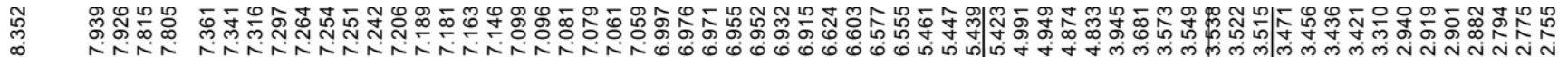

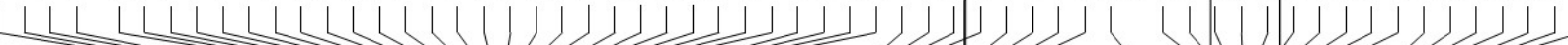<smiles>COc1ccc(Cn2c(CCc3ccccc3)nnc2NC(=O)CC(=O)NC(=O)/C(C#N)=C/c2cccs2)cc1</smiles>

III\| $\|$ III
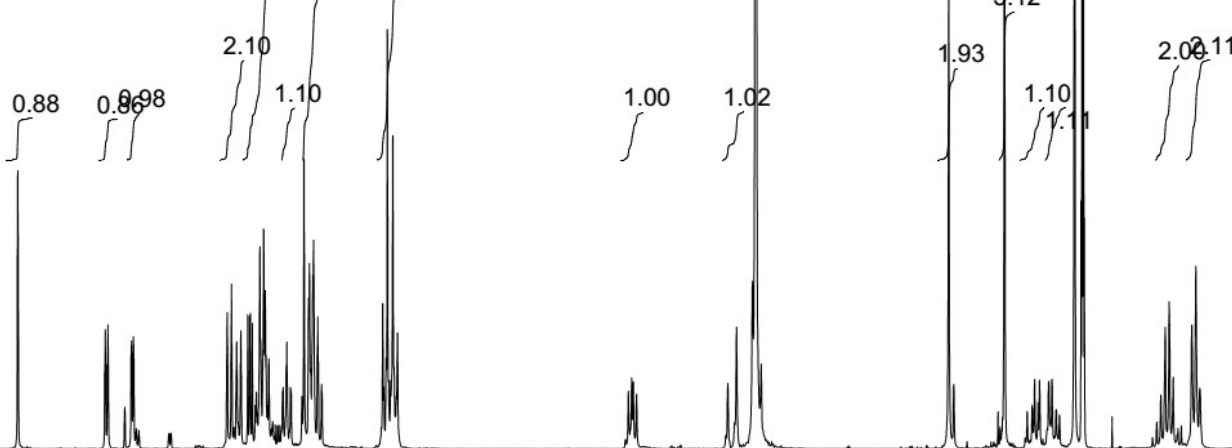

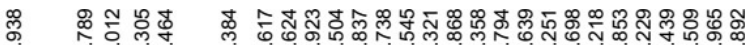

官

(1)
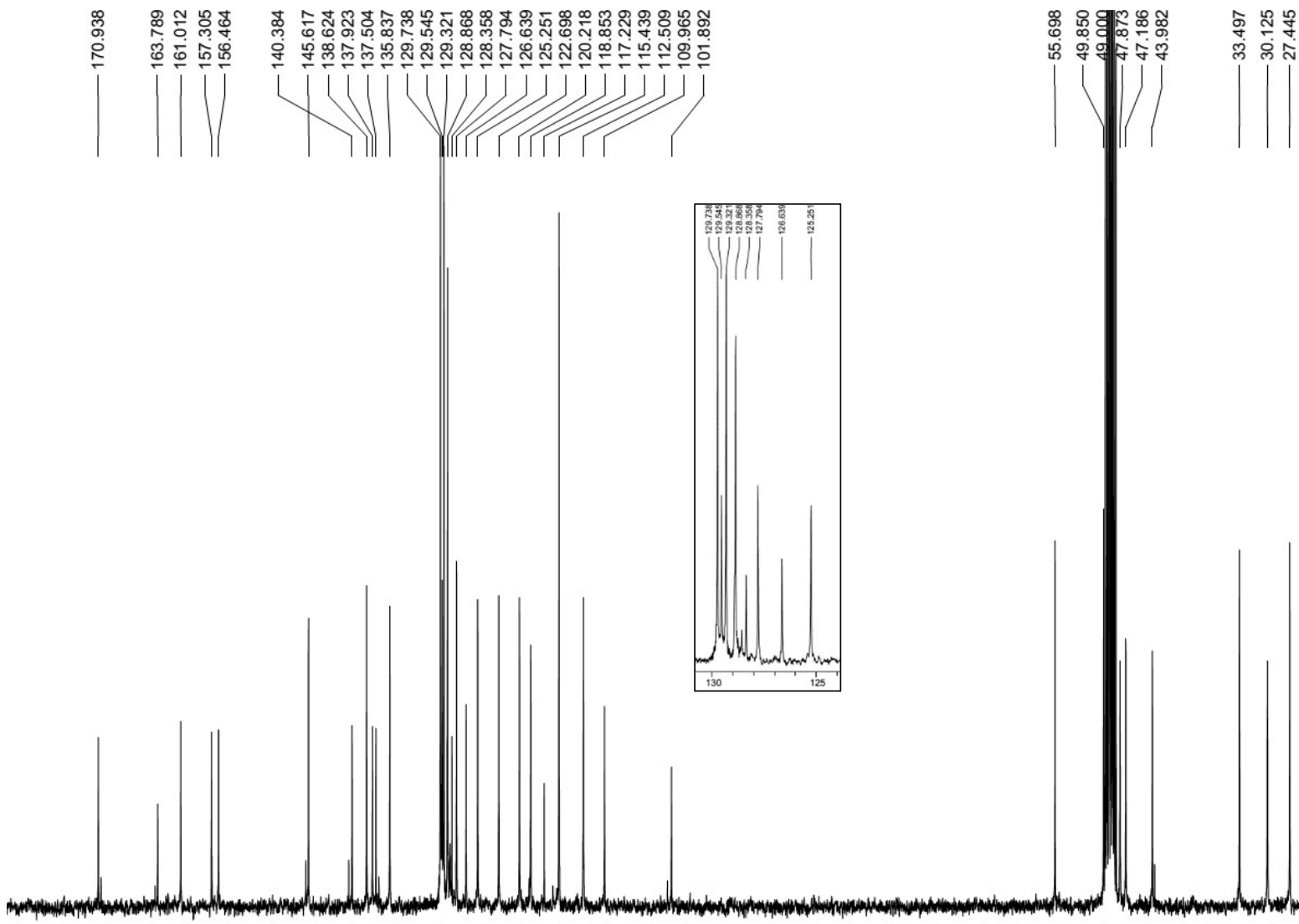
Compound 34

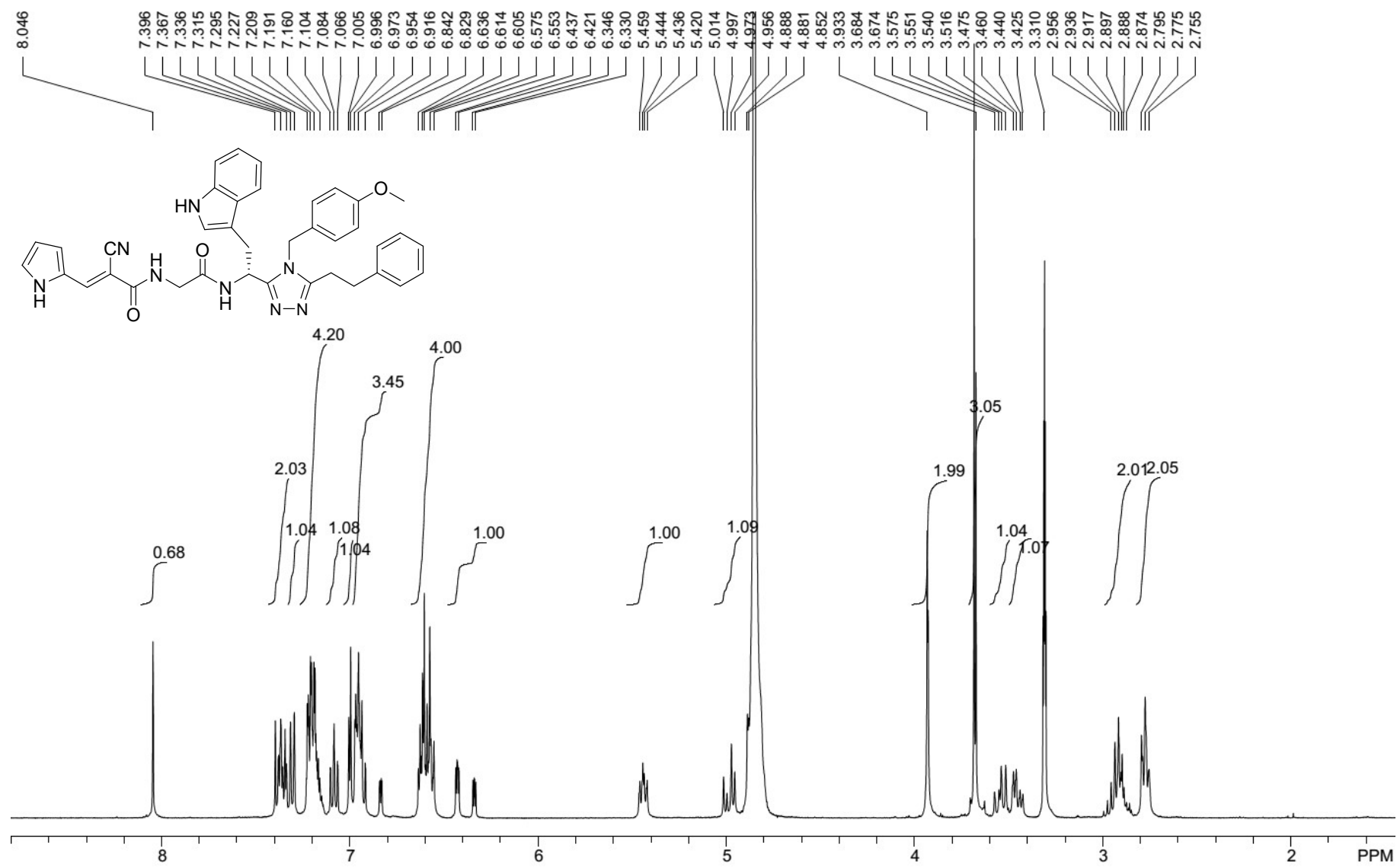

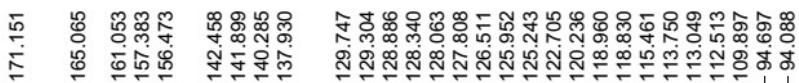

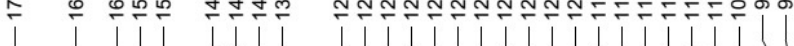

m
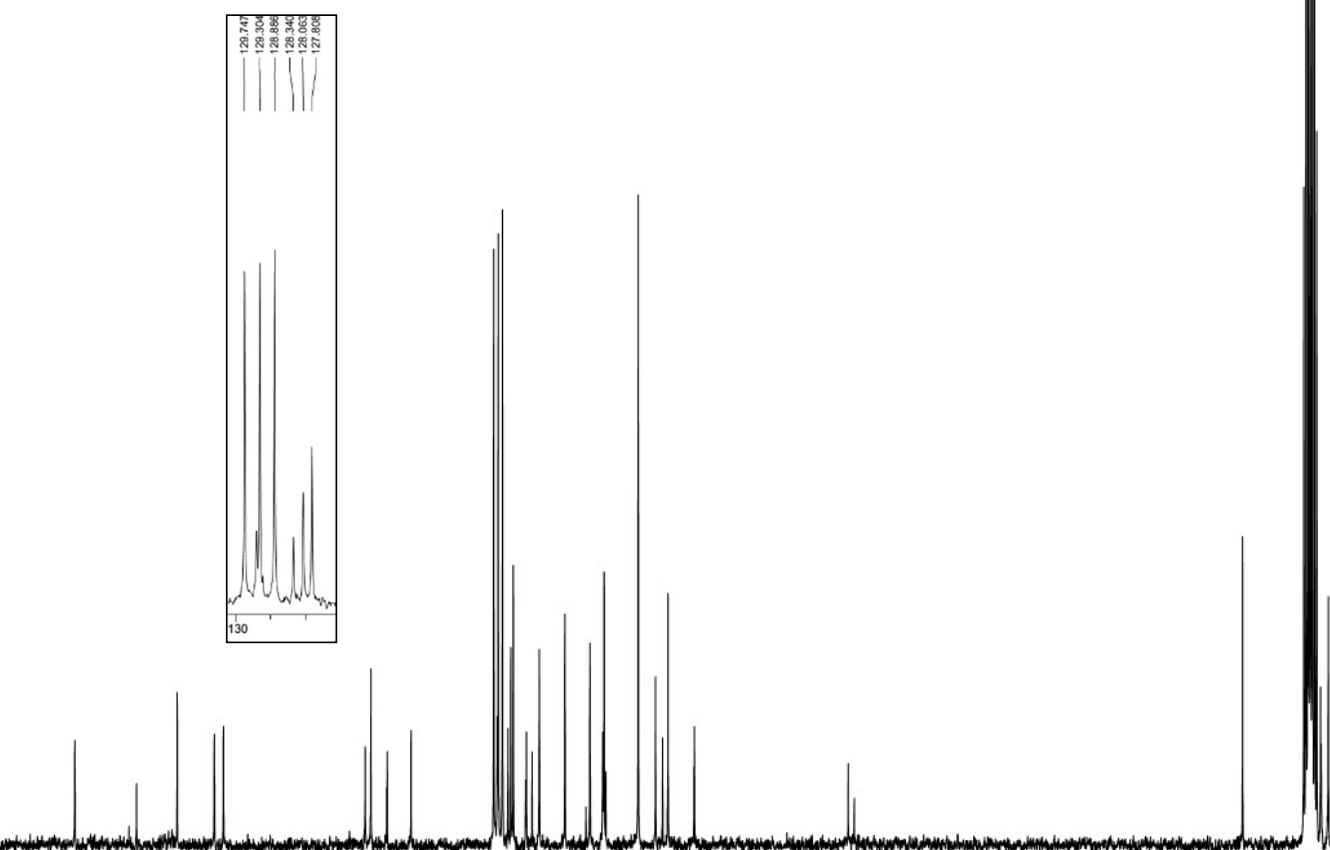
Compound 35
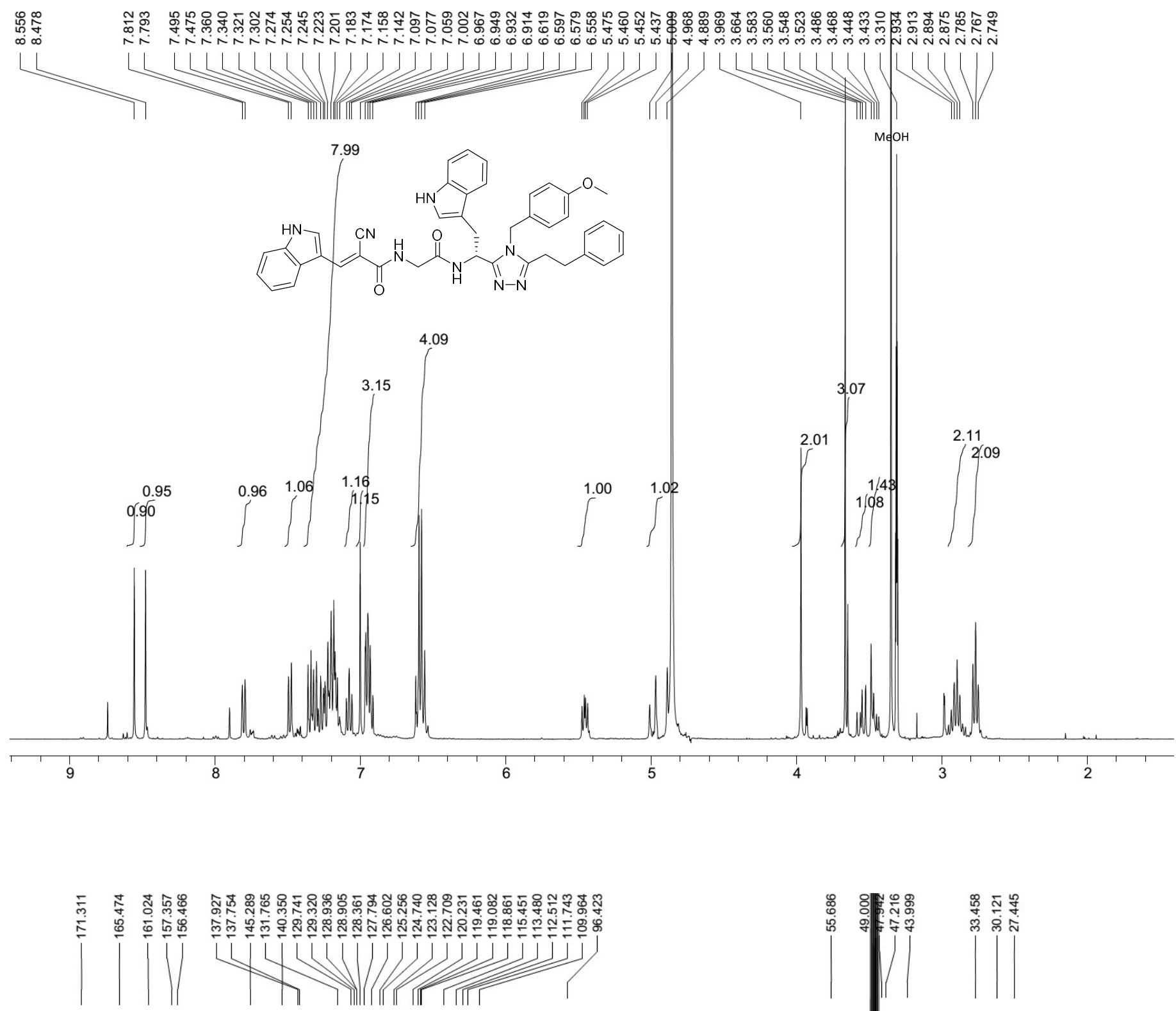


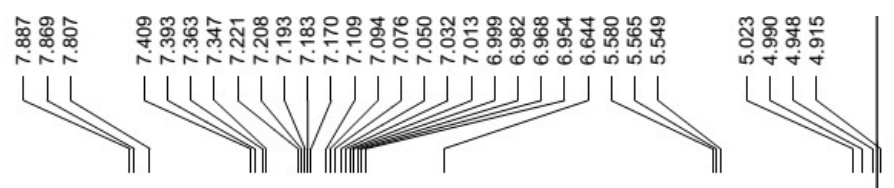

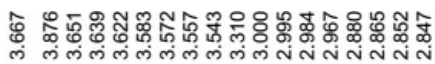

(1)
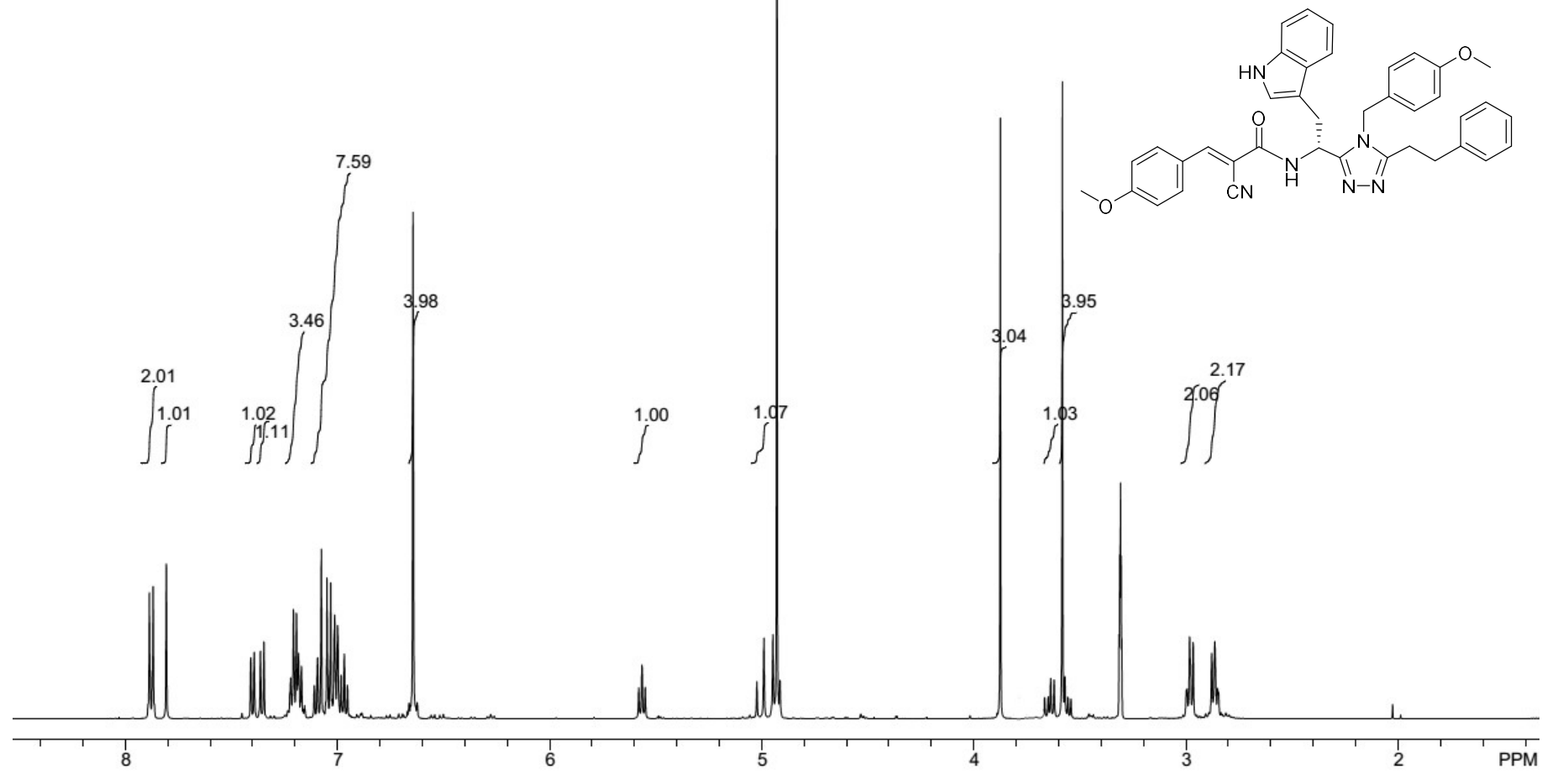

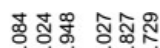

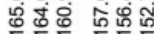

111

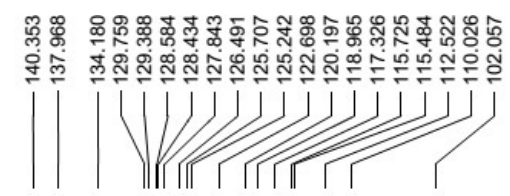

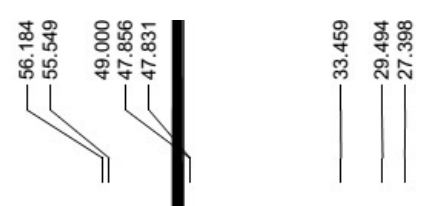

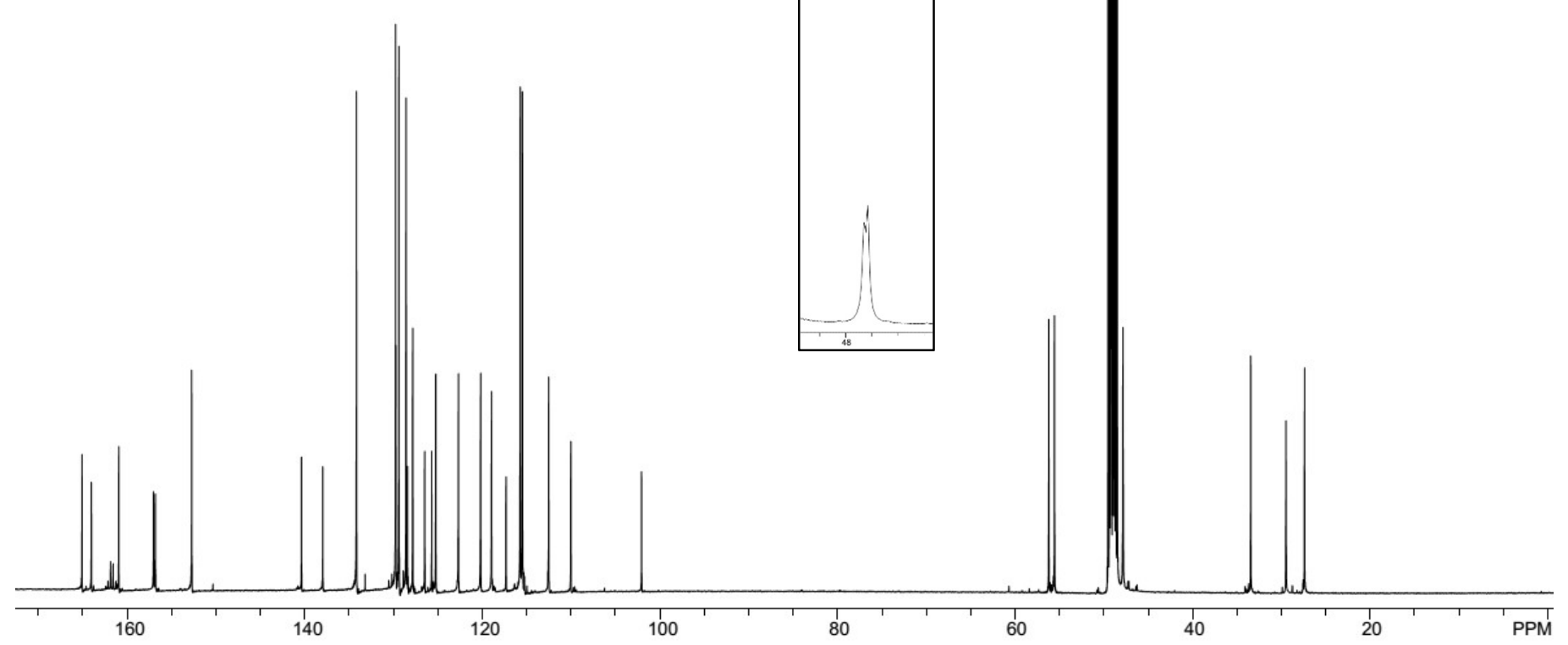


Compound 37
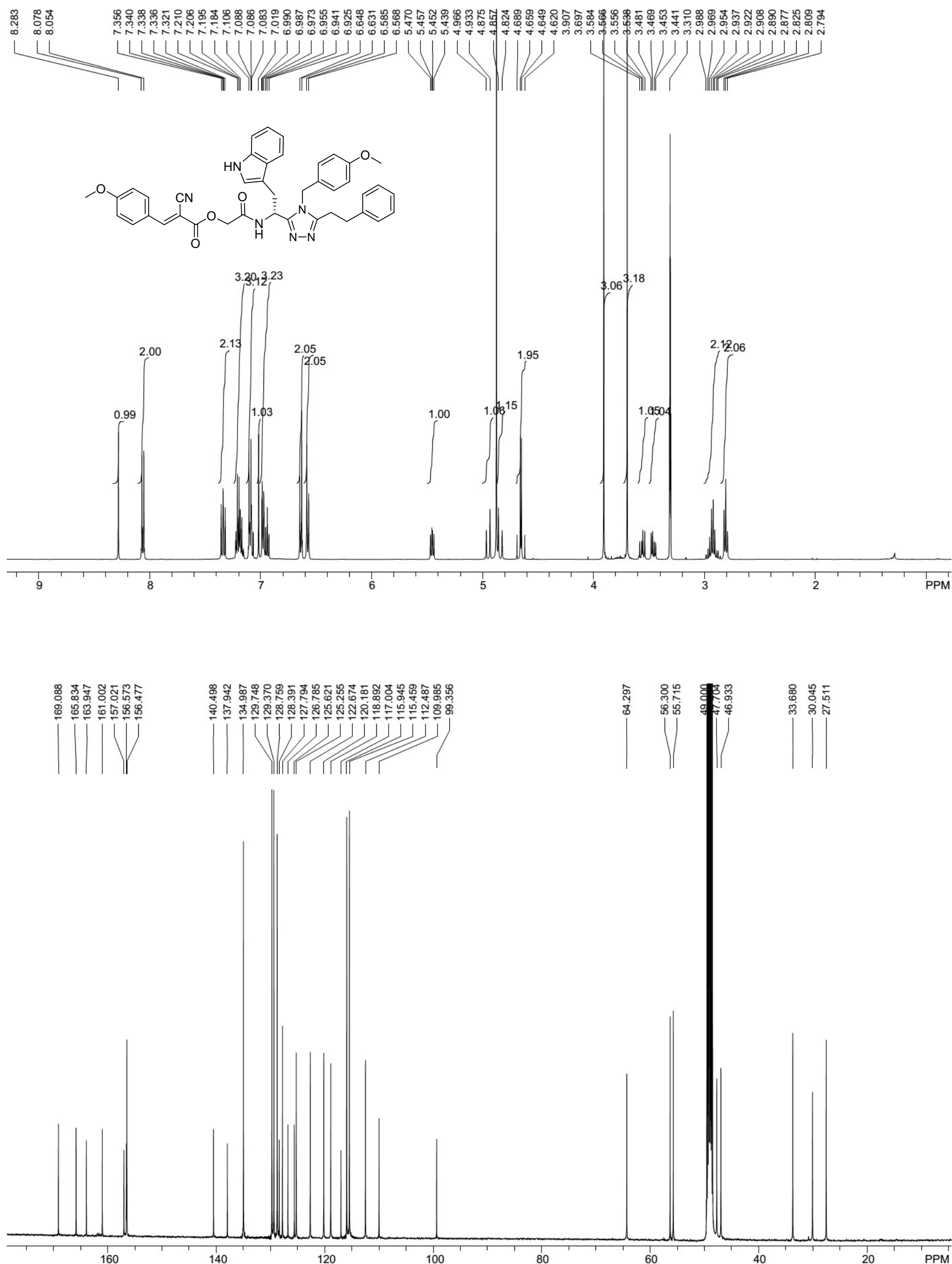
Compound $\mathbf{3 8}$
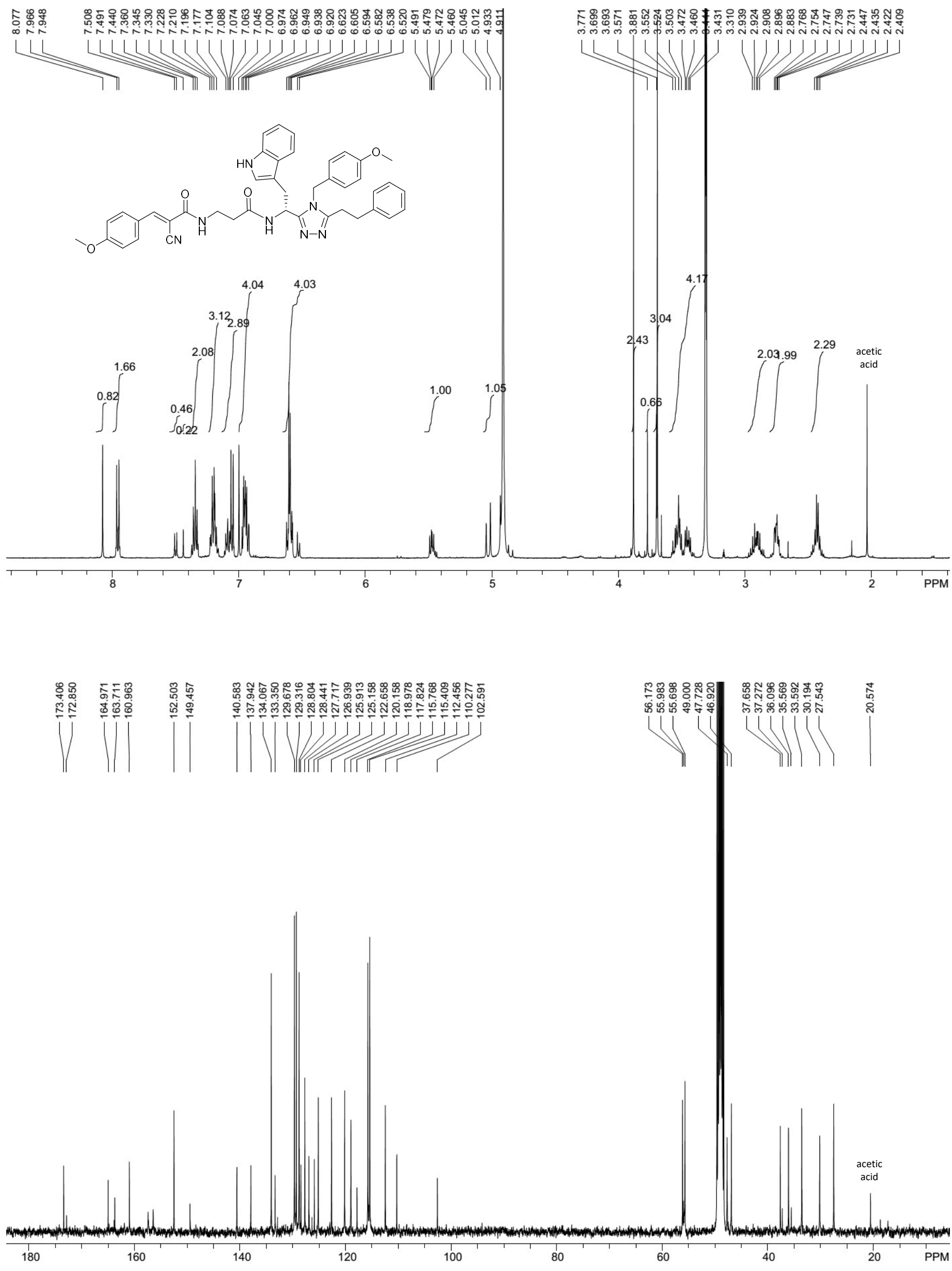
Compound 39

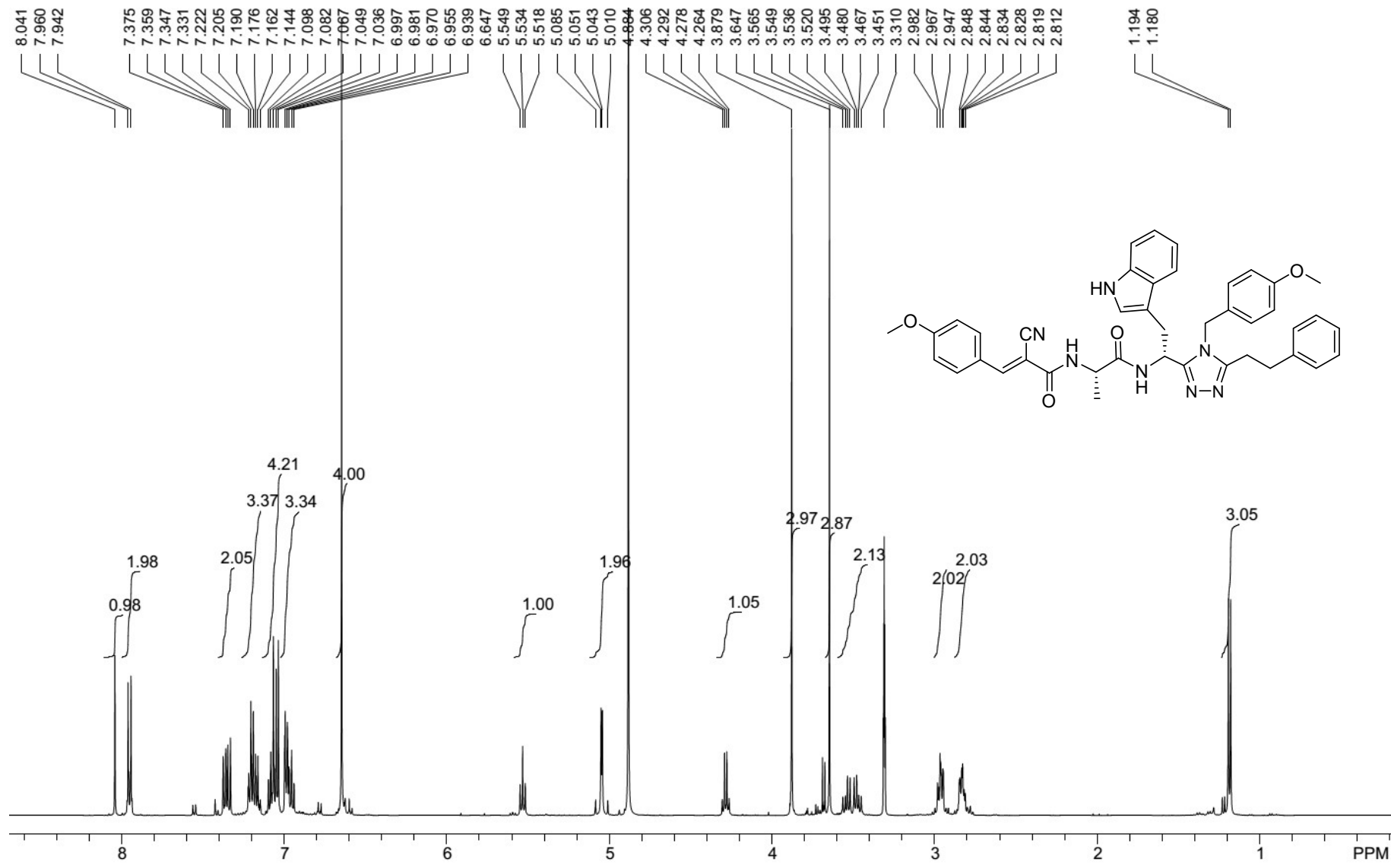

$\left.\right|_{\mid} ^{\infty}$

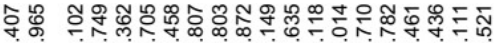

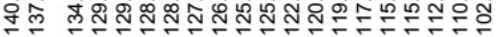
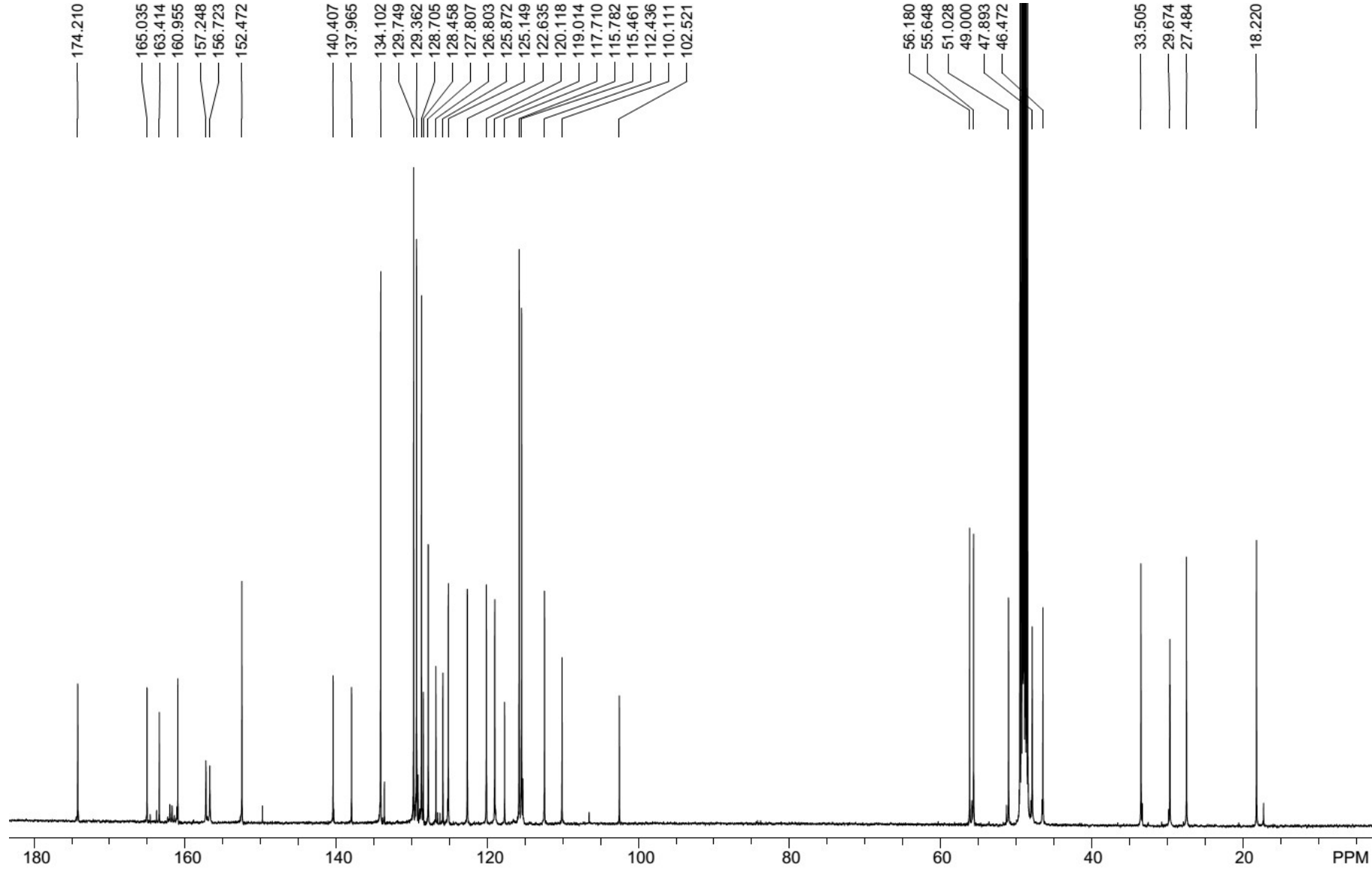


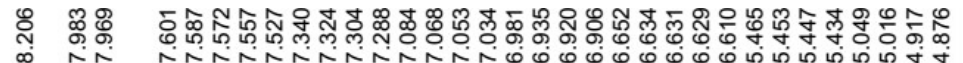

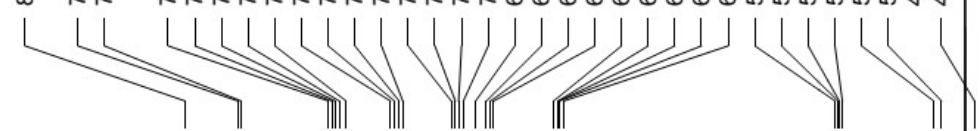

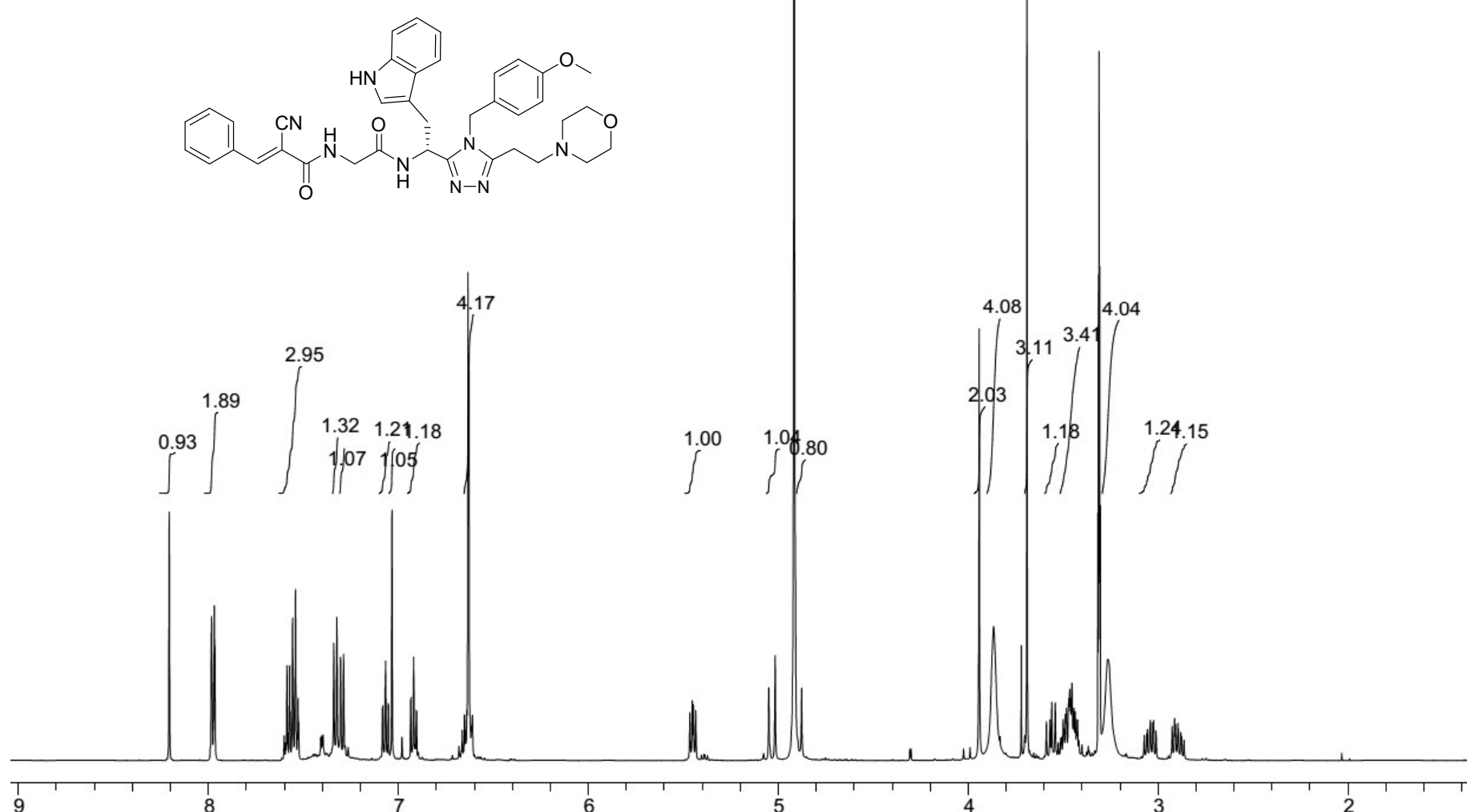

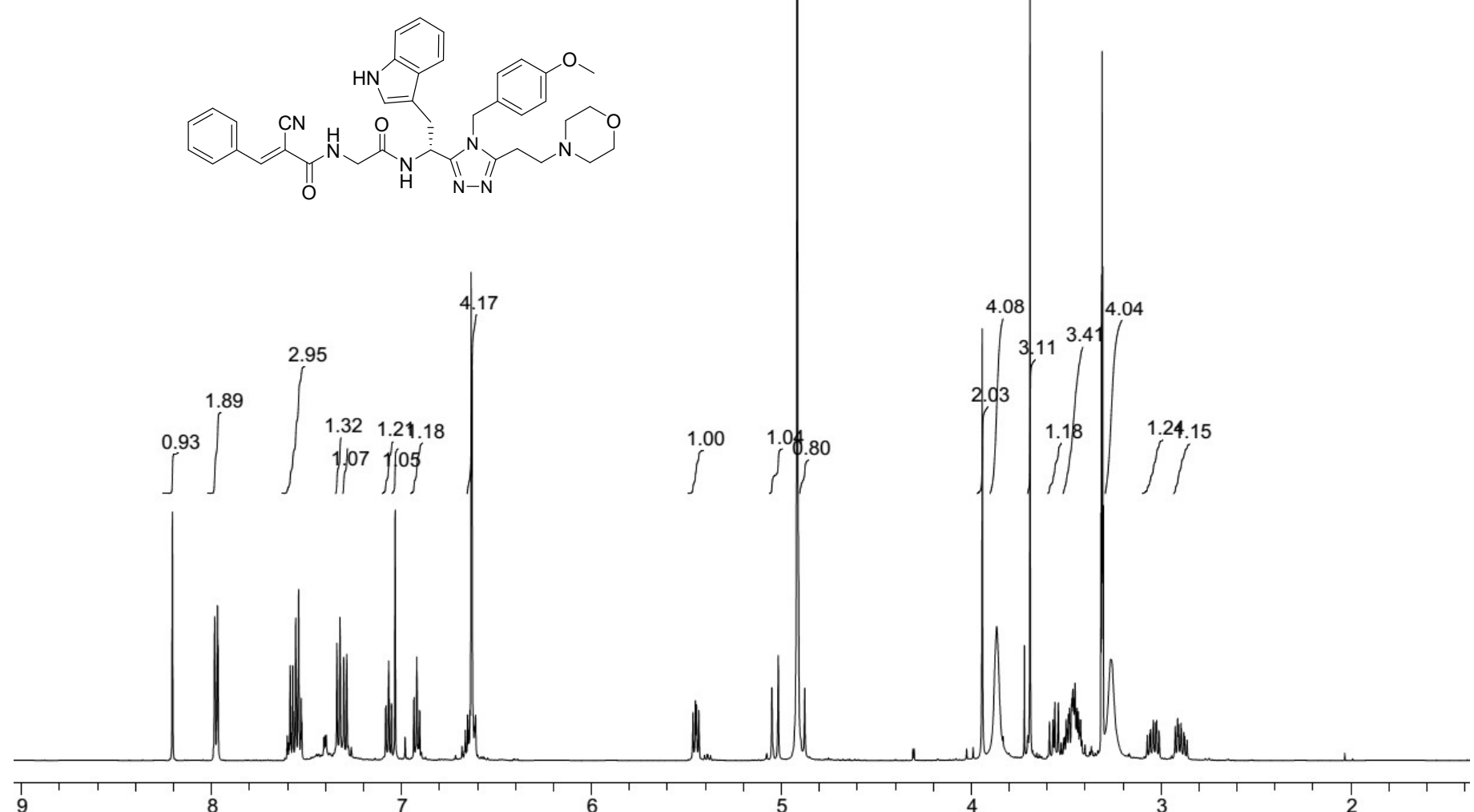

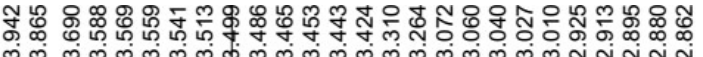

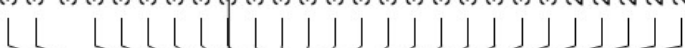
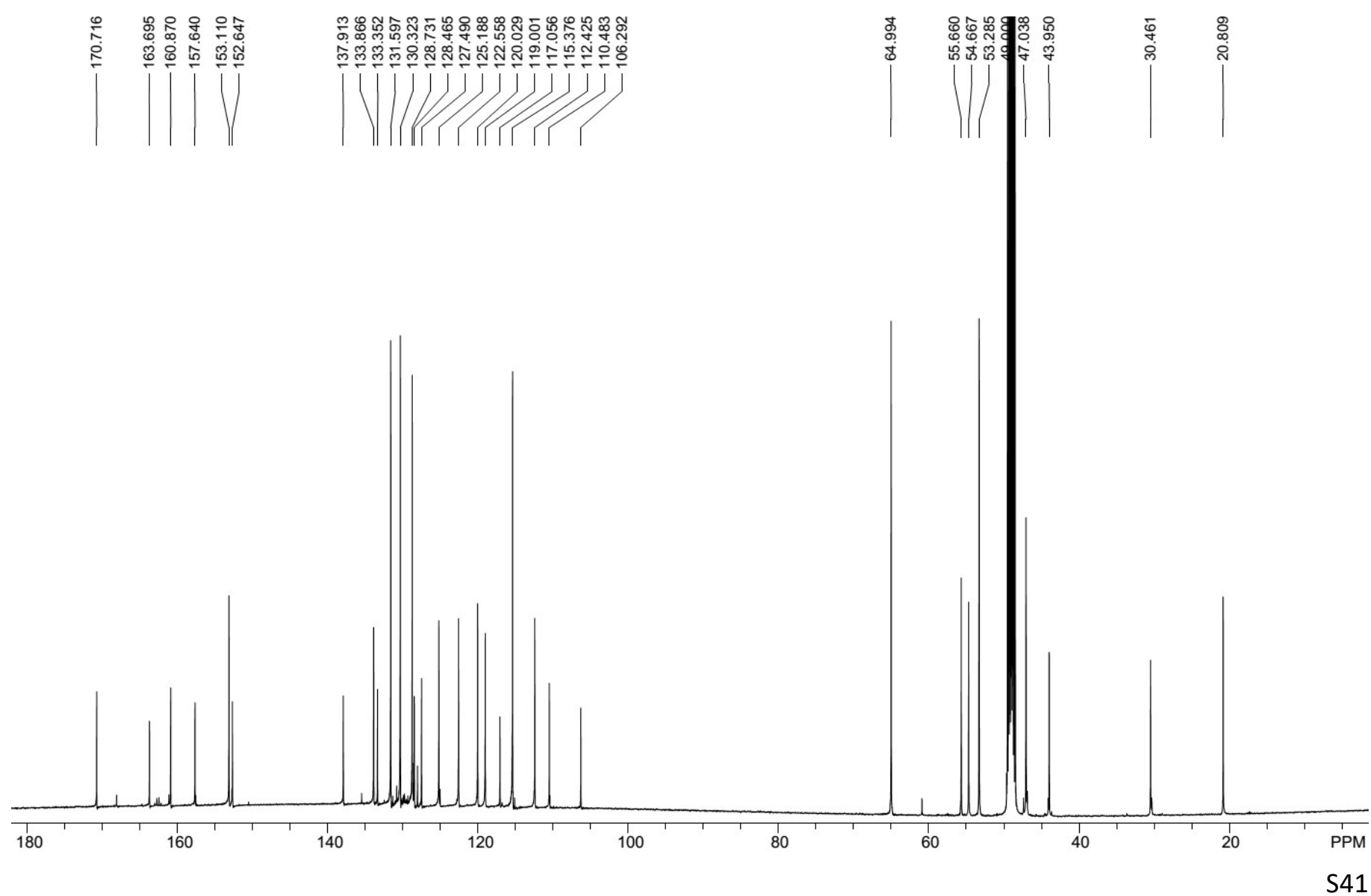
Compound 41

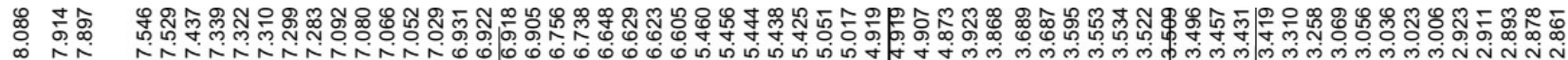

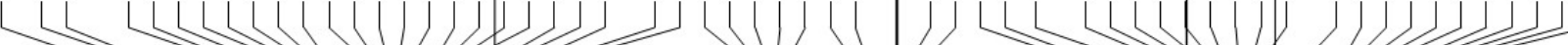

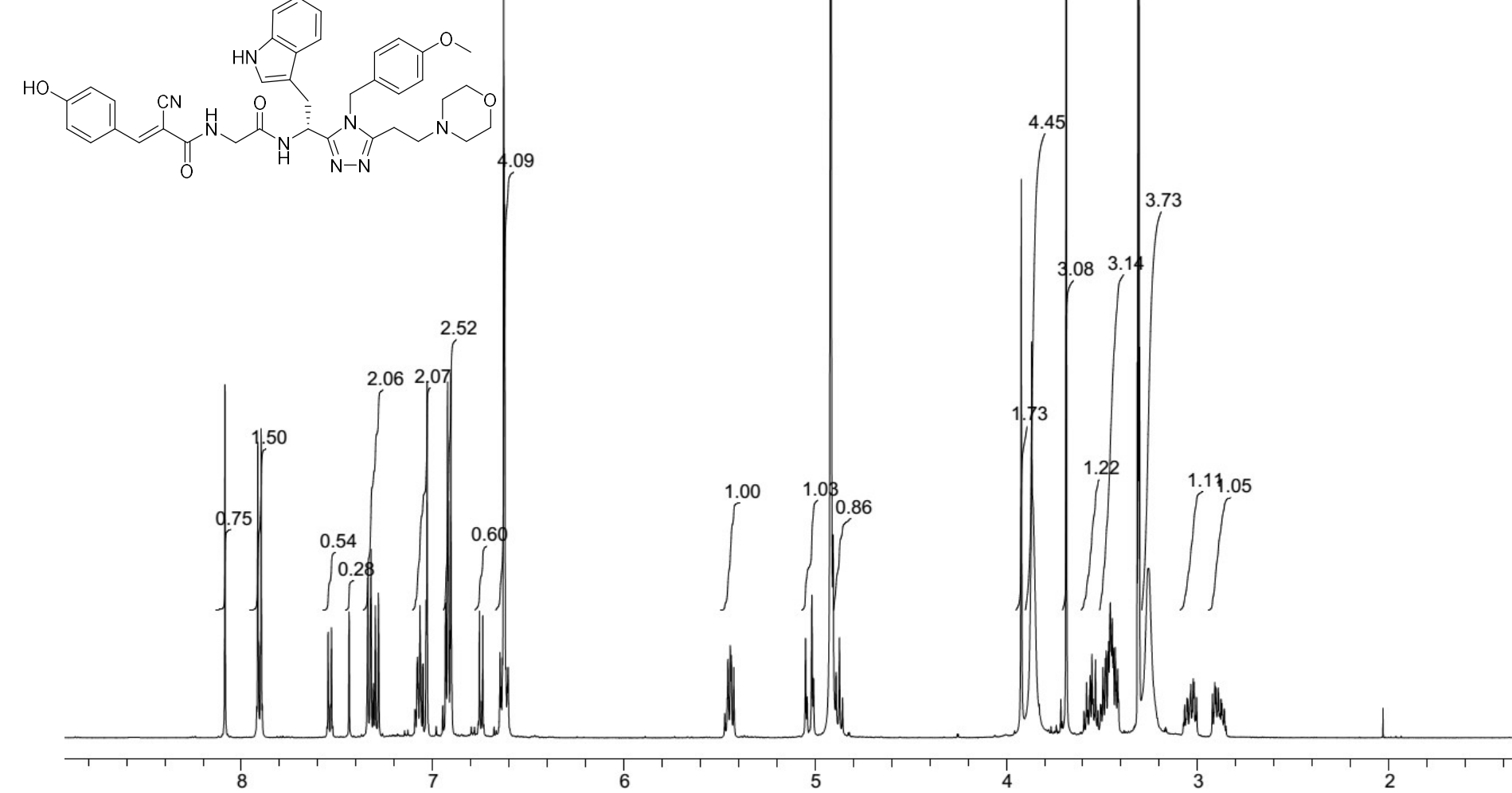

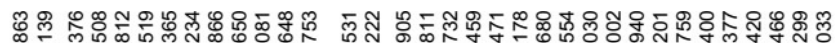

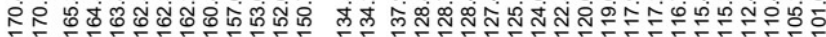
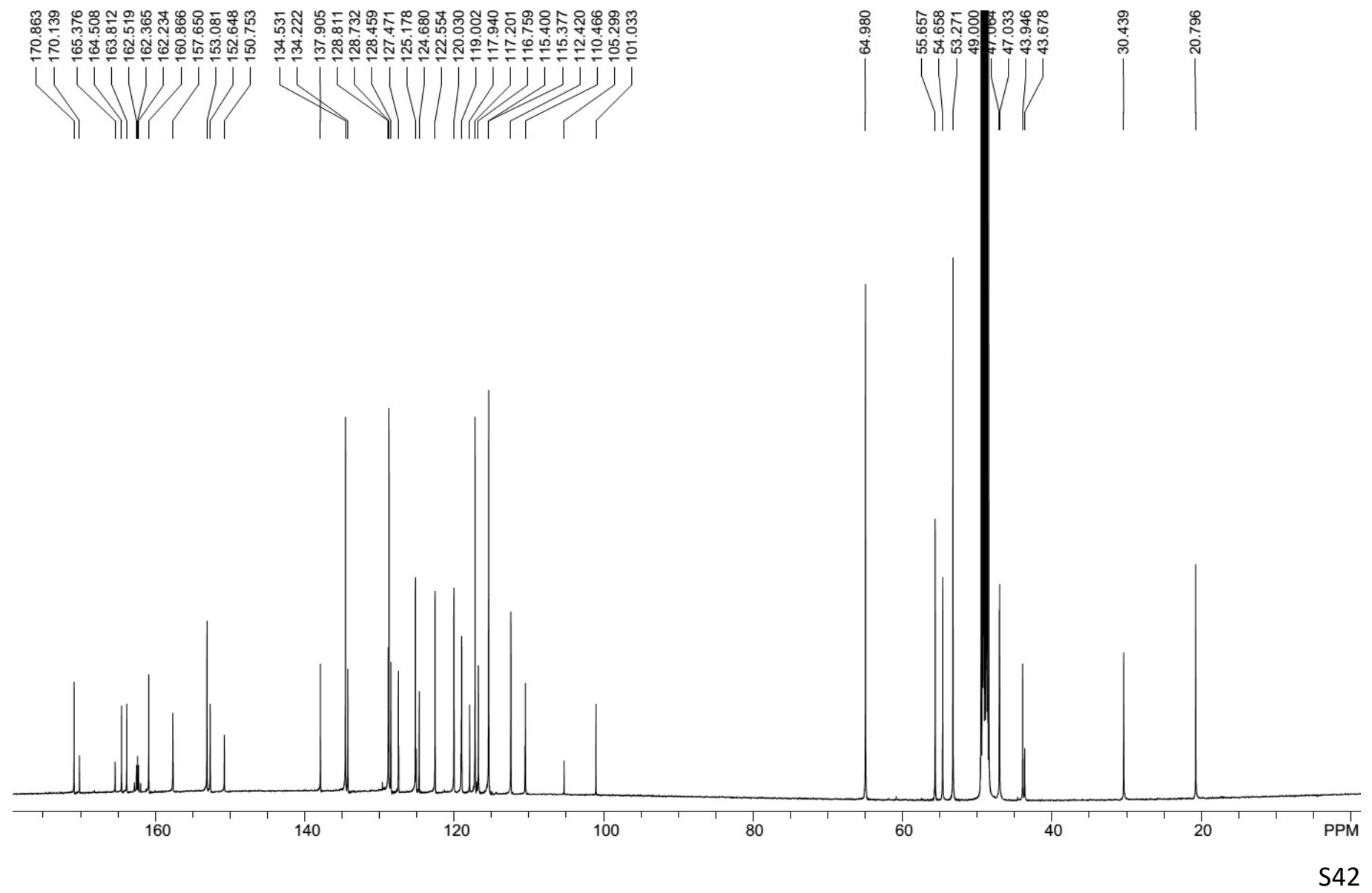


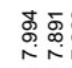
\î

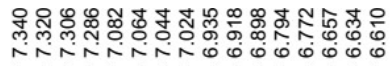
UVUVIU HI III III II
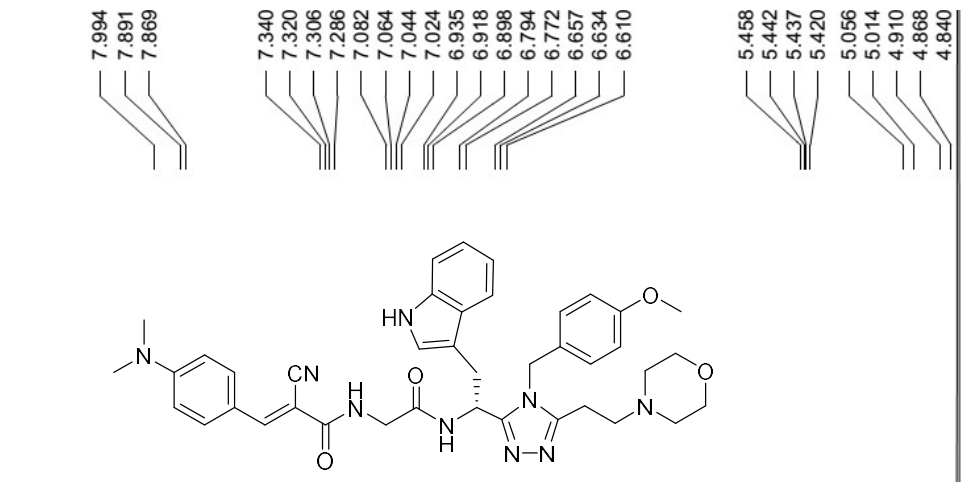

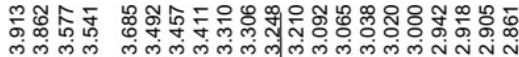

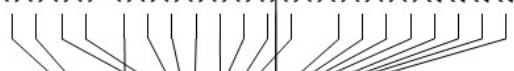

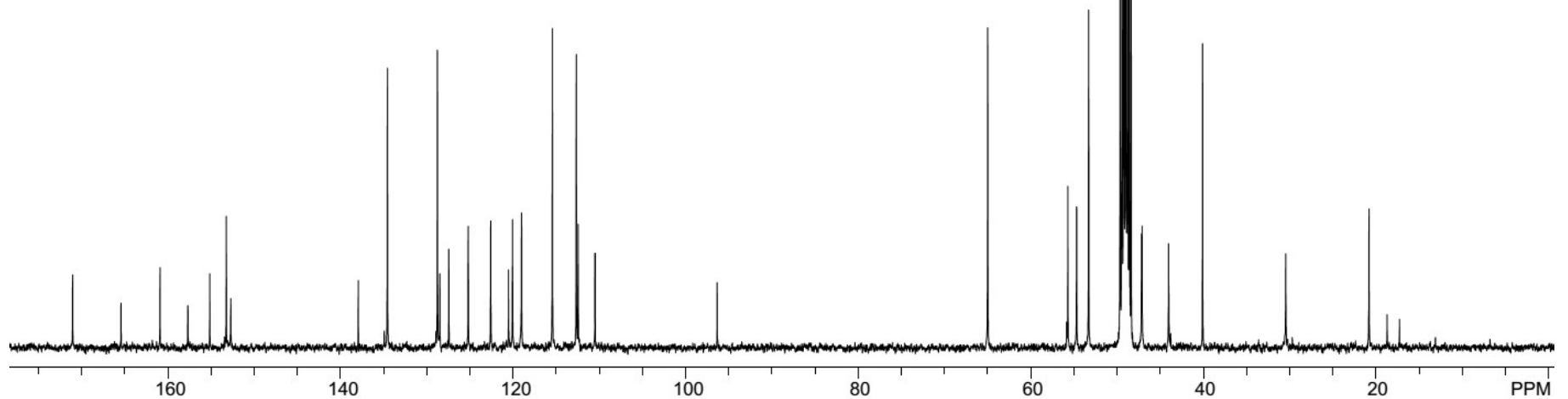


Compound 43

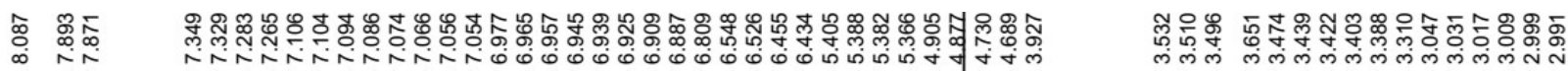

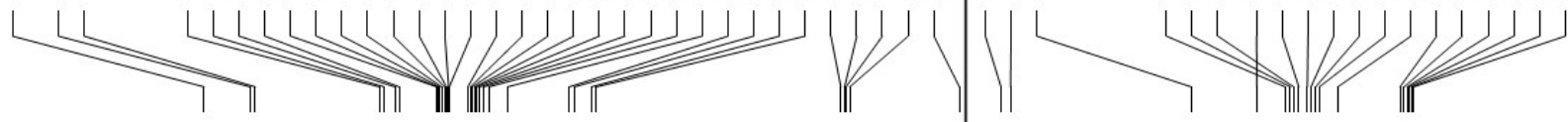

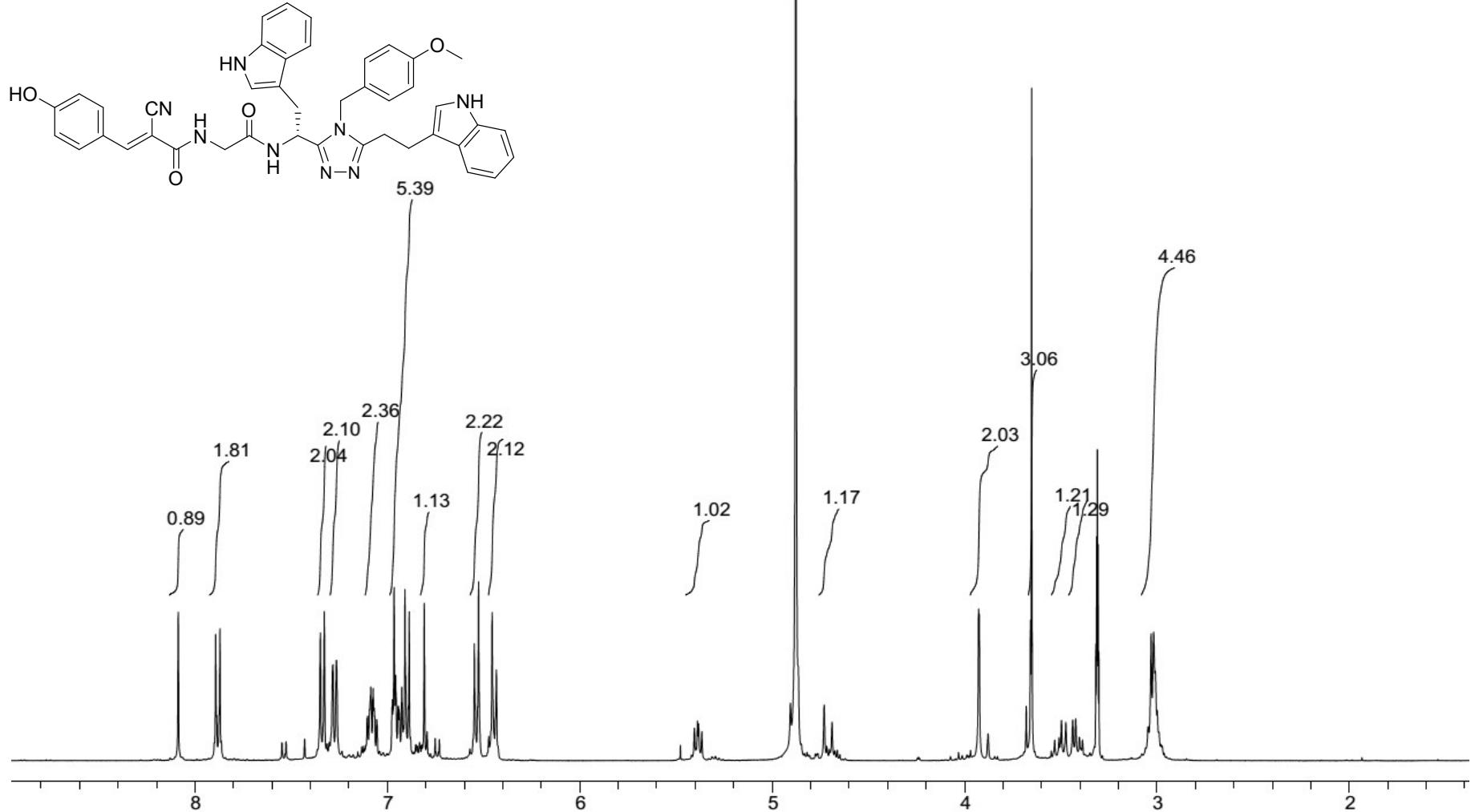

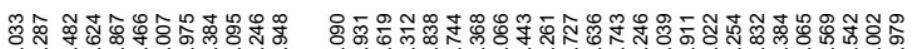

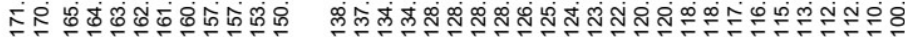

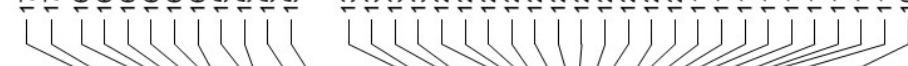

m M M M Mllinil

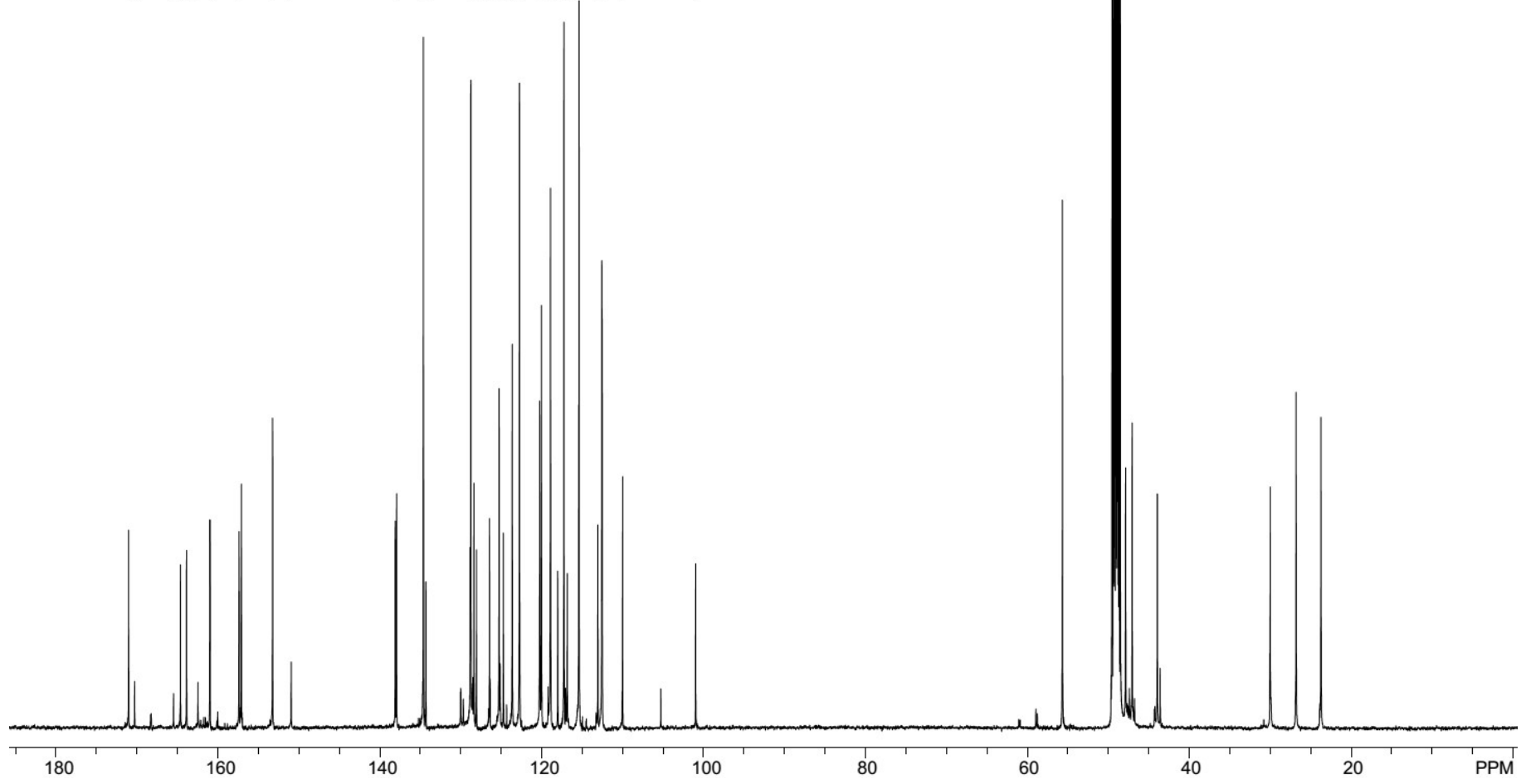


Compound 44

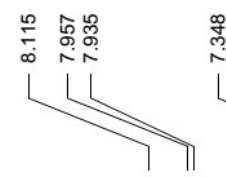

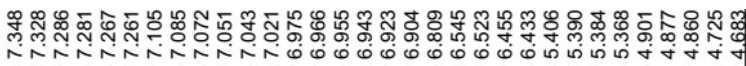

WU

"ौl Wini $\pi$

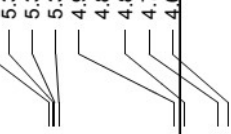

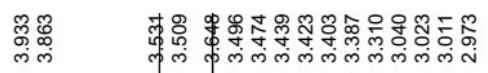

2

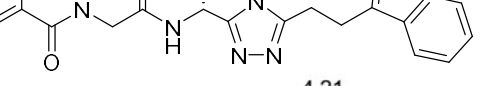

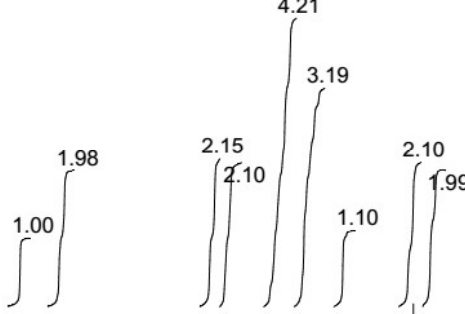

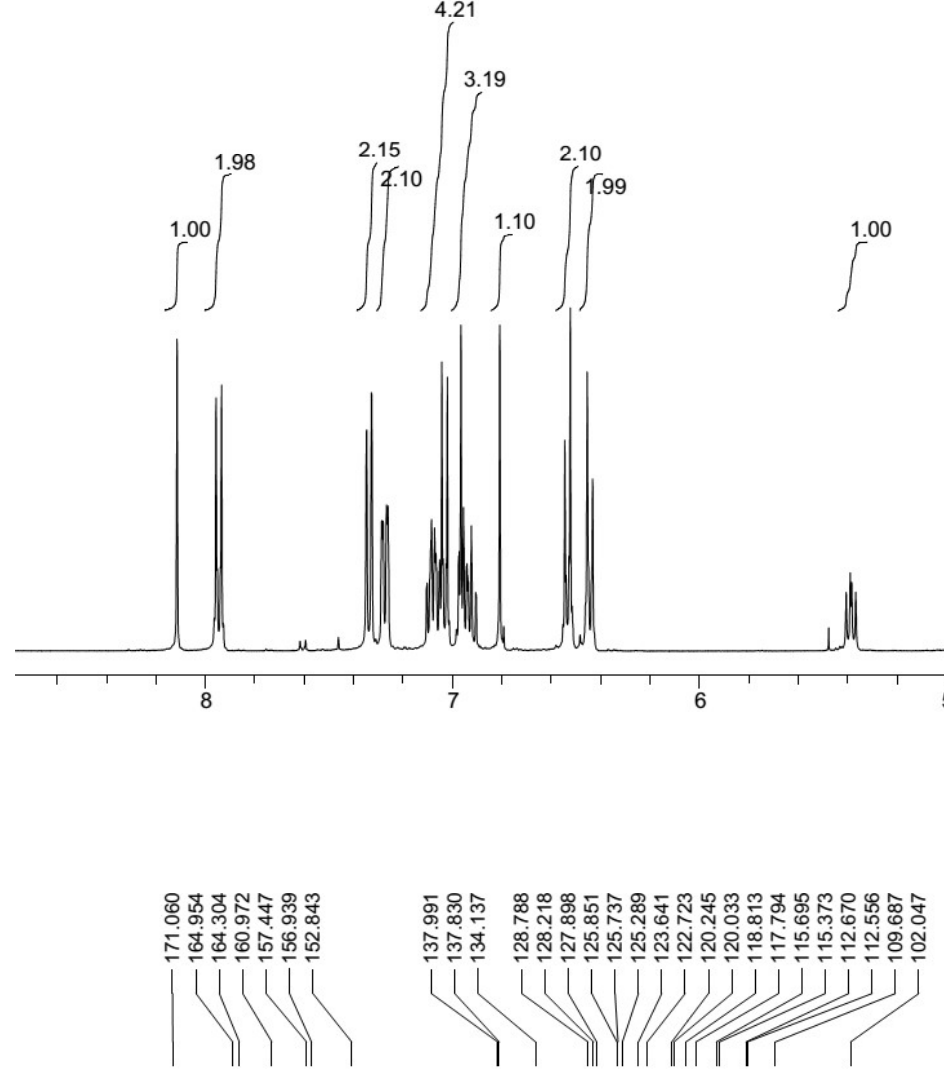

$\int^{1.00}$

$\int^{1.02}$
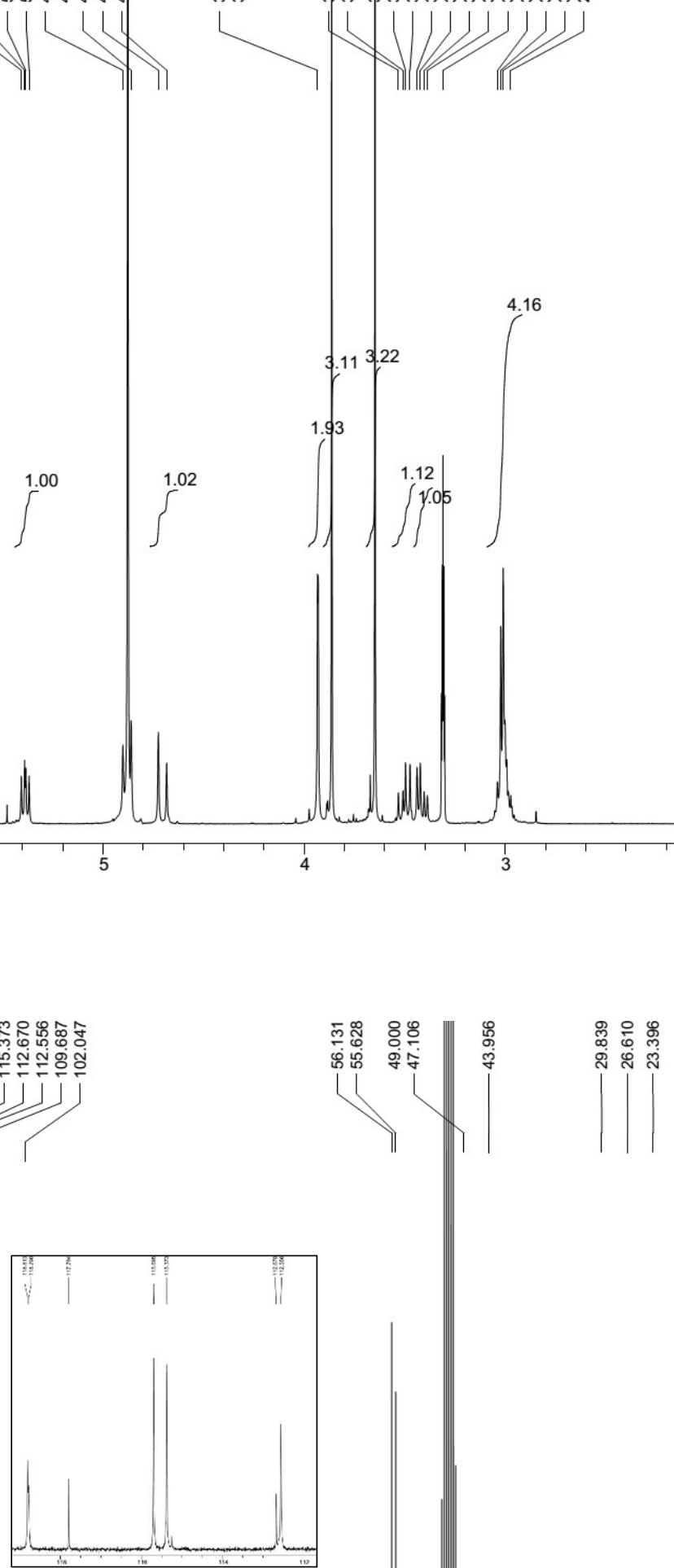


\section{Compound 45}

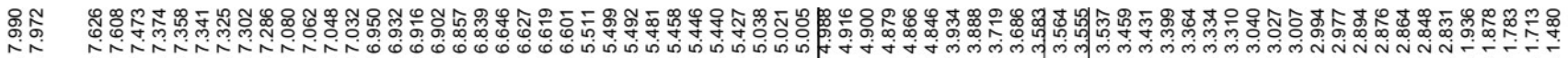

1 LU<smiles>COc1ccc(/C=C(\C)C(=O)NCC(=O)NCc2nnc(CCN3CCCCC3)n2Cc2ccc(OC)cc2)cc1</smiles>

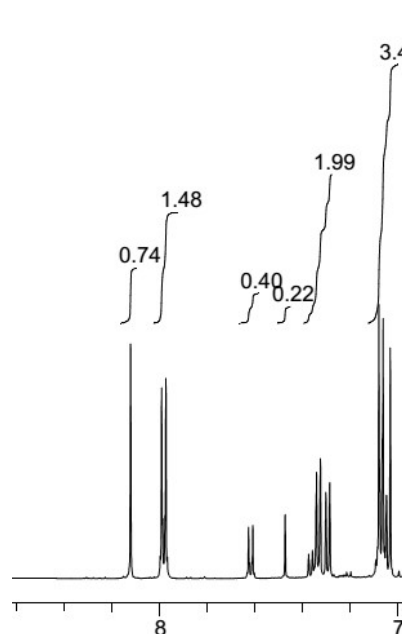

$3.48 \quad 3.85$

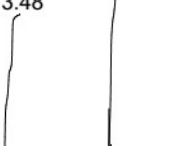

$0.74 \int^{1.48}$

1.19

$\iint^{0.49}$

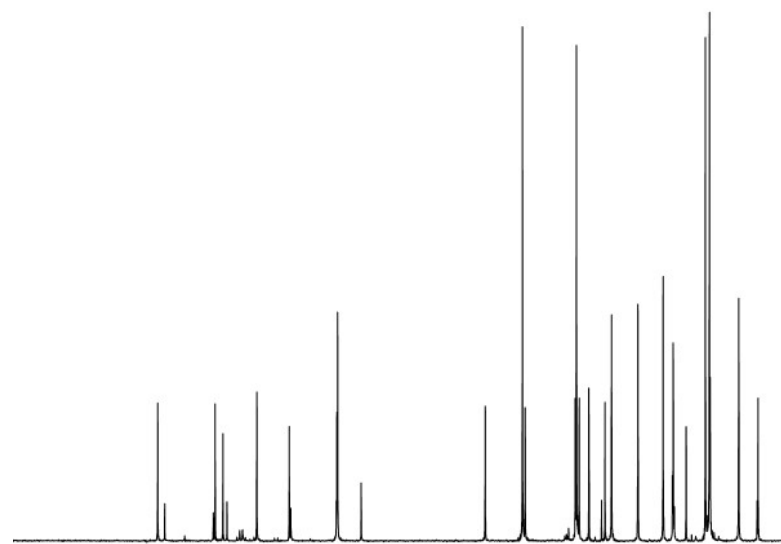




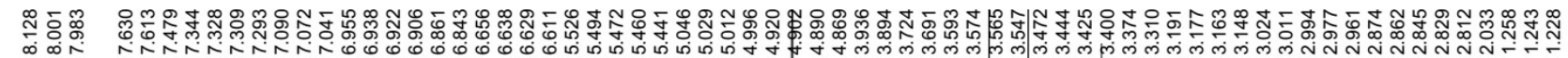

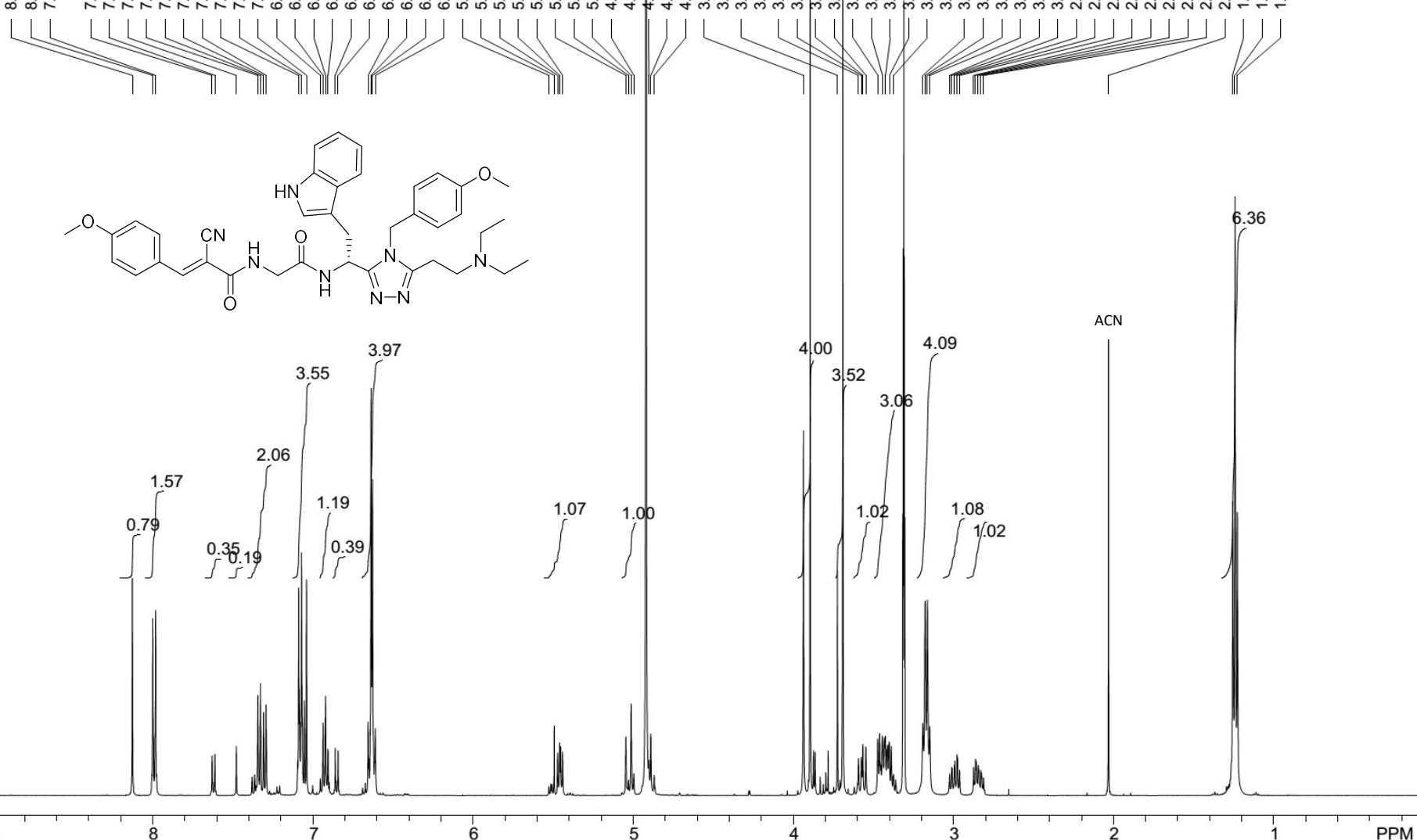

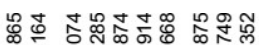
웅

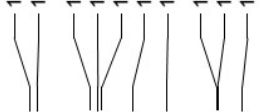

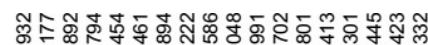

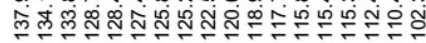

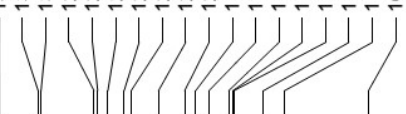

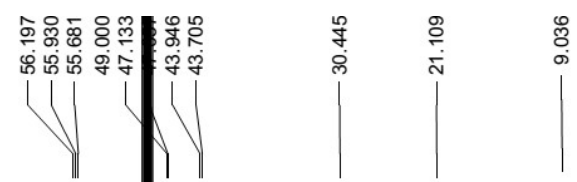

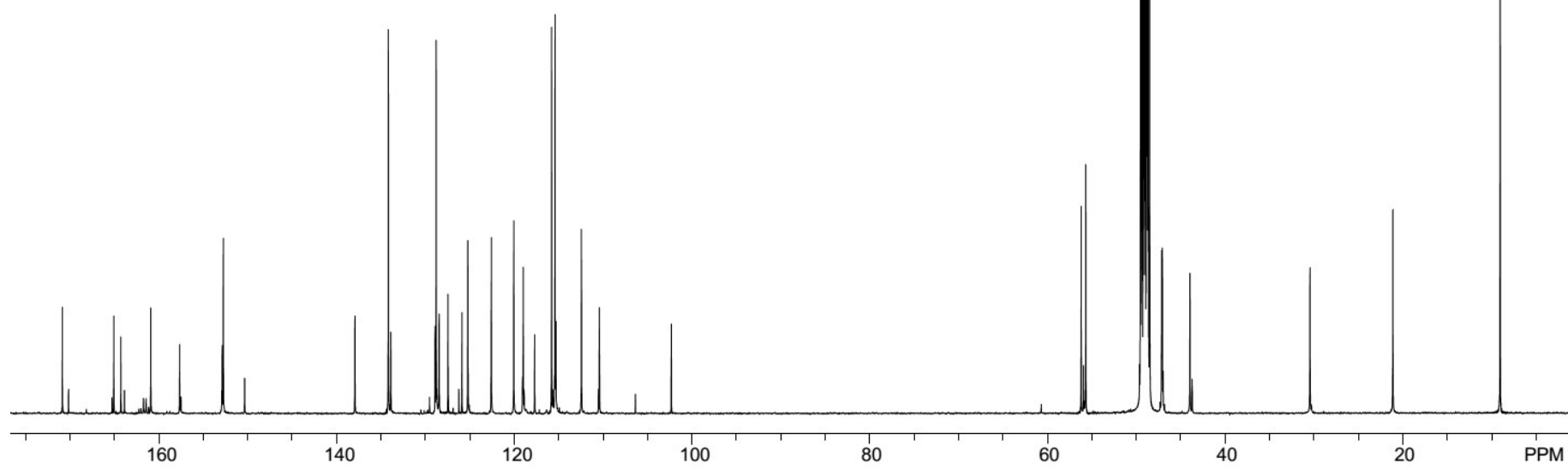


2. LC-MS chromatograms of key target ligands $27,29,30$ and 44

Ligand 27 (JAF 16-34)

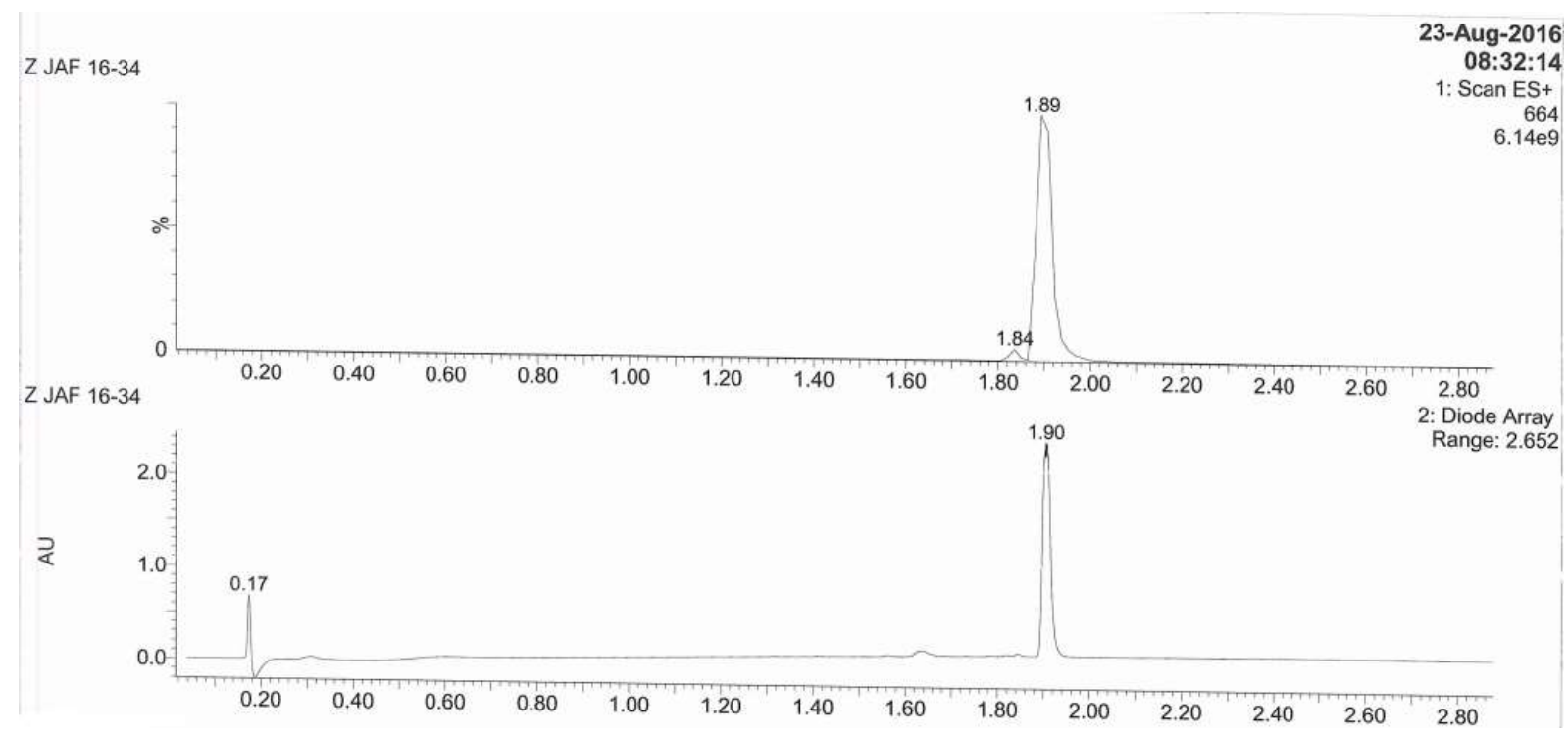

Ligand 29 (LM 01)

\section{ZQ routine}

Z LM 01 erlen3
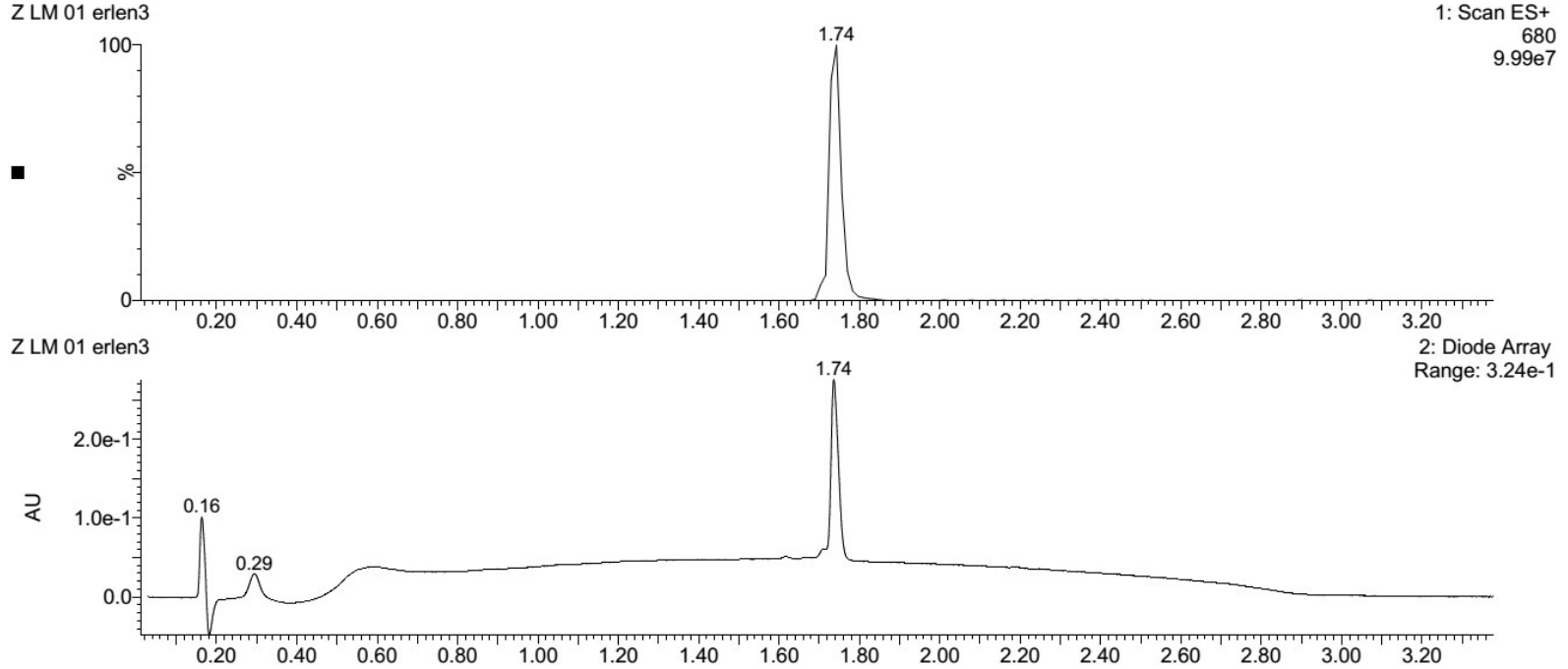


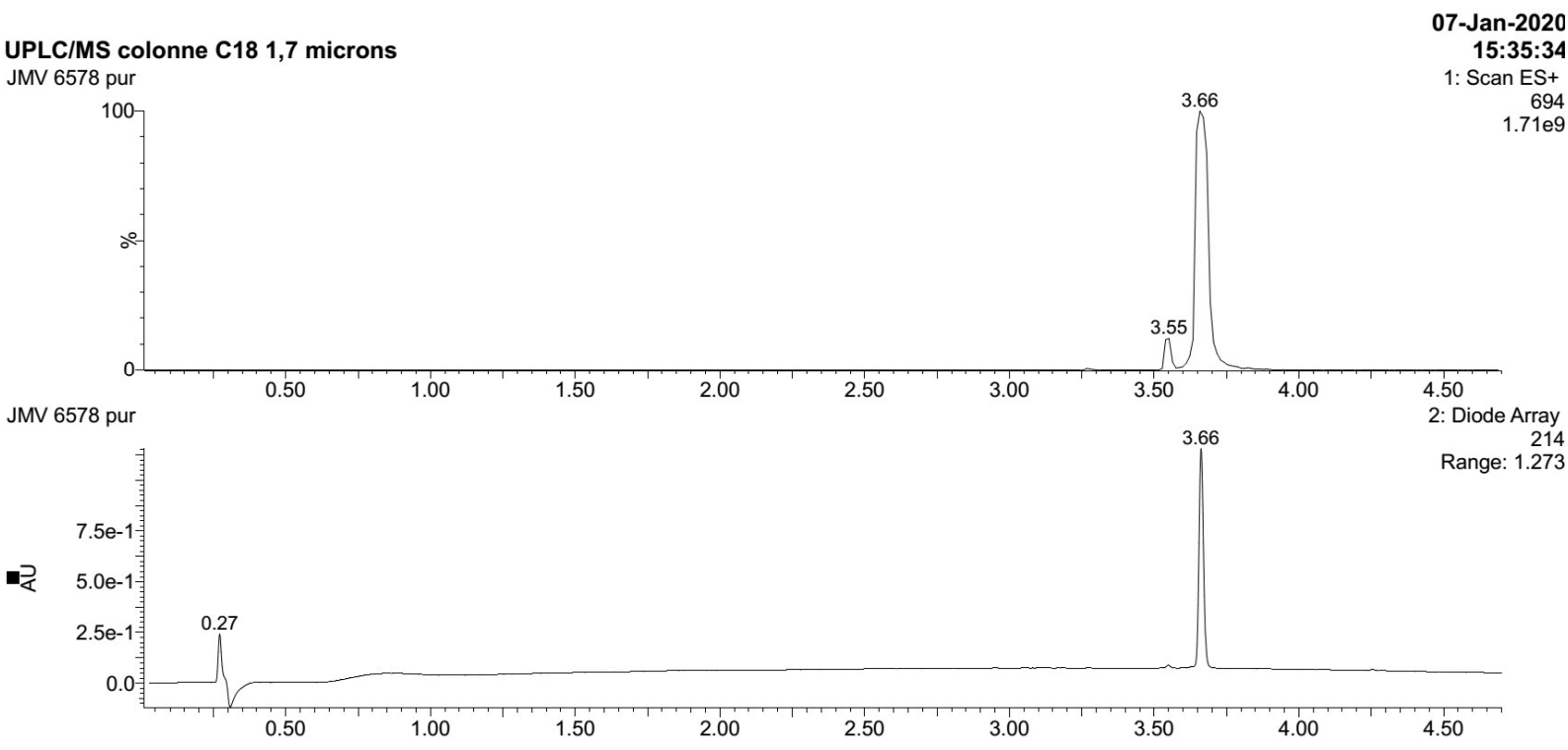

Ligand 44 (KHB 244)

Z KHB 244

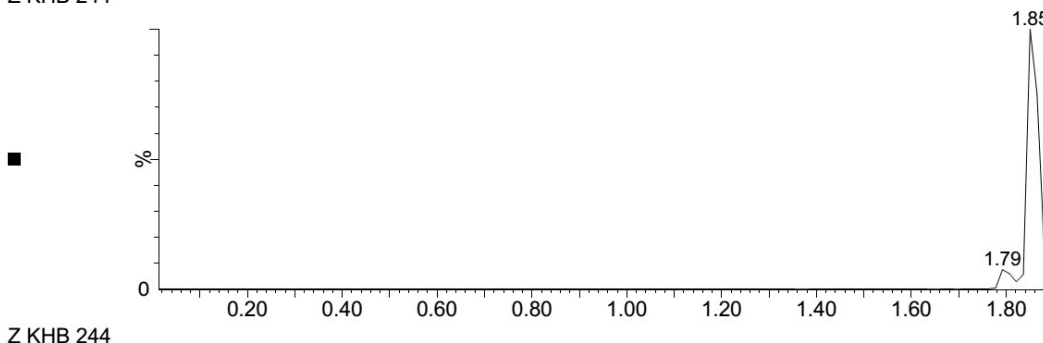

27-Sep-2016 13:27:43 1: Scan ES+ 733
$1.57 \mathrm{e} 9$

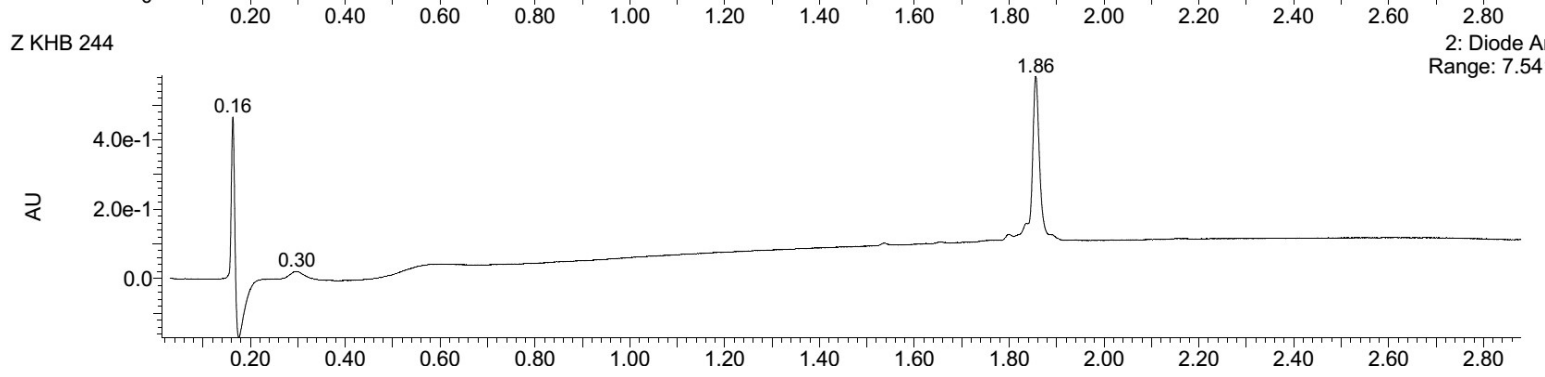


3.1. Preparation of the calibration curve in the range of $25-150 \mu \mathrm{M}$ in methanol

\begin{tabular}{|c|c|}
\hline Conc. $(\mu \mathrm{M})$ & AUC $(214 \mathrm{~nm})$ \\
\hline 25 & 7.14359 \\
\hline 50 & 13.30683 \\
\hline 100 & 25.21906 \\
\hline 150 & 36.60163 \\
\hline
\end{tabular}

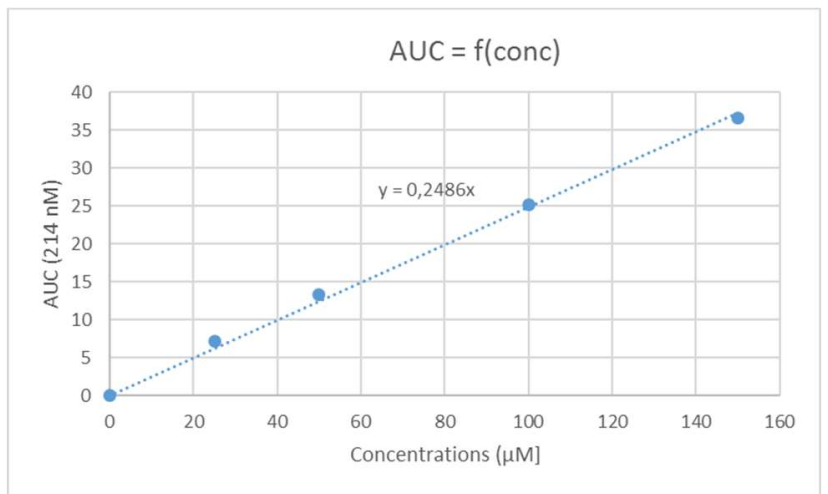

3.2. Preparation of solutions of ligand $\mathbf{2 7}$ in water

Solution 1: $1 \mathrm{mg}$ of ligand 27 in $500 \mu \mathrm{L}$ of water

Solution 2: $1 \mathrm{mg}$ of ligand $27 \mathrm{in} 1 \mathrm{~mL}$ of water

Protocol:

- $1 \mathrm{mg}$ of ligand 27 is solubilized in $\mathrm{x} \mathrm{mL}$ of water $(\mathrm{x}=500 \mu \mathrm{L}$ or $1 \mathrm{~mL})$

- after $5 \mathrm{~min}$ of wortexing, the solution is put under sonication at $25^{\circ} \mathrm{C}$ during $15 \mathrm{~min}$ (this step is done twice)

- the solution is centrifuged at $3000 \mathrm{rpm}$ during $10 \mathrm{~min}$

- $20 \mu \mathrm{L}$ of the supernatant are collected and injected in an analytical HPLC apparatus (reverse phase)

3.3. Results

\begin{tabular}{|c|c|c|}
\hline Solutions & AUC $(214 \mathrm{~nm})$ & Corresponding conc. $(\mu \mathrm{M})$ \\
\hline 1 & 2.01275 & 8.1 \\
\hline 2 & 1.86896 & 7.5 \\
\hline
\end{tabular}

Water solubility of ligand $27: 5.2 \mathrm{mg} / \mathrm{L}$ 
4. Determination of the water solubility of ligand $\mathbf{2 9}$

4.1. Preparation of the calibration curve in the range of $50-200 \mu \mathrm{M}$ in methanol

\begin{tabular}{|c|c|}
\hline Conc. $(\mu \mathrm{M})$ & AUC $(214 \mathrm{~nm})$ \\
\hline 50 & 12.56731 \\
\hline 100 & 26.32178 \\
\hline 150 & 38.95833 \\
\hline 200 & 54.13637 \\
\hline
\end{tabular}

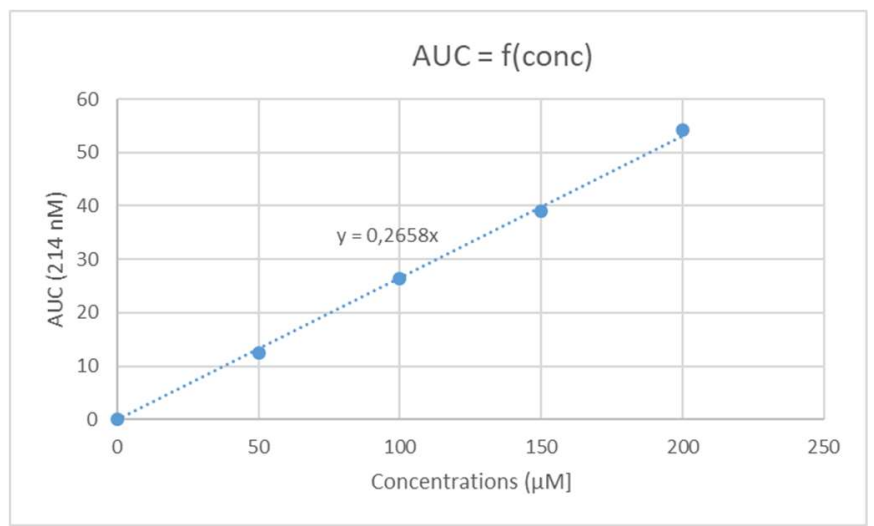

4.2. Preparation of solutions of ligand $\mathbf{2 9}$ in water

Solution 1: $1 \mathrm{mg}$ of ligand 29 in $1 \mathrm{~mL}$ of water

Solution 2: $1 \mathrm{mg}$ of ligand 29 in $10 \mathrm{~mL}$ of water

Protocol:

- $1 \mathrm{mg}$ of ligand $\mathbf{2 9}$ is solubilized in $\mathrm{x} \mathrm{mL}$ of water $(\mathrm{x}=1$ or $10 \mathrm{~mL})$

- after 5 min of wortexing, the solution is put under sonication at $25^{\circ} \mathrm{C}$ during $15 \mathrm{~min}$ (this step is done twice)

- the solution is centrifuged at $3000 \mathrm{rpm}$ during $10 \mathrm{~min}$

- $20 \mu \mathrm{L}$ of the supernatant are collected and injected in an analytical HPLC apparatus (reverse phase)

4.3. Results

\begin{tabular}{|c|c|c|}
\hline Solutions & AUC $(214 \mathrm{~nm})$ & Corresponding conc. $(\mu \mathrm{M})$ \\
\hline 1 & 9.80878 & 36.9 \\
\hline 2 & 9.54284 & 35.9 \\
\hline
\end{tabular}

Water solubility of ligand 29: $24.7 \mathrm{mg} / \mathrm{L}$ 
5. Determination of the water solubility of ligand $\mathbf{3 0}$

5.1. Preparation of the calibration curve in the range of $25-150 \mu \mathrm{M}$ in methanol

\begin{tabular}{|c|c|}
\hline Conc. $(\mu \mathrm{M})$ & AUC $(214 \mathrm{~nm})$ \\
\hline 25 & 6.63143 \\
\hline 50 & 12.99132 \\
\hline 100 & 25.32611 \\
\hline 150 & 36.17371 \\
\hline
\end{tabular}

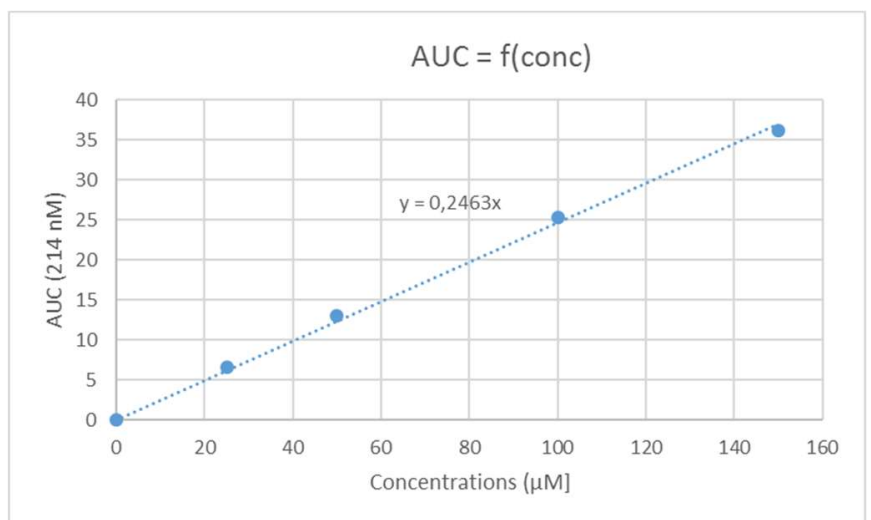

5.2. Preparation of solutions of ligand $\mathbf{3 0}$ in water

Solution 1: $1 \mathrm{mg}$ of ligand $\mathbf{3 0}$ in $500 \mu \mathrm{L}$ of water

Solution 2: $1 \mathrm{mg}$ of ligand $\mathbf{3 0}$ in $1 \mathrm{~mL}$ of water

Protocol:

- $1 \mathrm{mg}$ of ligand $\mathbf{3 0}$ is solubilized in $\mathrm{x} \mathrm{mL}$ of water $(\mathrm{x}=500 \mu \mathrm{L}$ or $1 \mathrm{~mL})$

- after $5 \mathrm{~min}$ of wortexing, the solution is put under sonication at $25^{\circ} \mathrm{C}$ during $15 \mathrm{~min}$ (this step is done twice)

- the solution is centrifuged at $3000 \mathrm{rpm}$ during $10 \mathrm{~min}$

- $20 \mu \mathrm{L}$ of the supernatant are collected and injected in an analytical HPLC apparatus (reverse phase)

5.3. Results

\begin{tabular}{|c|c|c|}
\hline Solutions & AUC $(214 \mathrm{~nm})$ & Corresponding conc. $(\mu \mathrm{M})$ \\
\hline 1 & 2.56656 & 10.4 \\
\hline 2 & 2.37734 & 9.6 \\
\hline
\end{tabular}

Water solubility of ligand 30: $6.9 \mathrm{mg} / \mathrm{L}$ 
6.1. Preparation of the calibration curve in the range of $25-150 \mu \mathrm{M}$ in methanol

\begin{tabular}{|c|c|}
\hline Conc. $(\mu \mathrm{M})$ & AUC $(214 \mathrm{~nm})$ \\
\hline 25 & 10.53563 \\
\hline 50 & 19.62200 \\
\hline 100 & 39.47924 \\
\hline 150 & 54.89496 \\
\hline
\end{tabular}

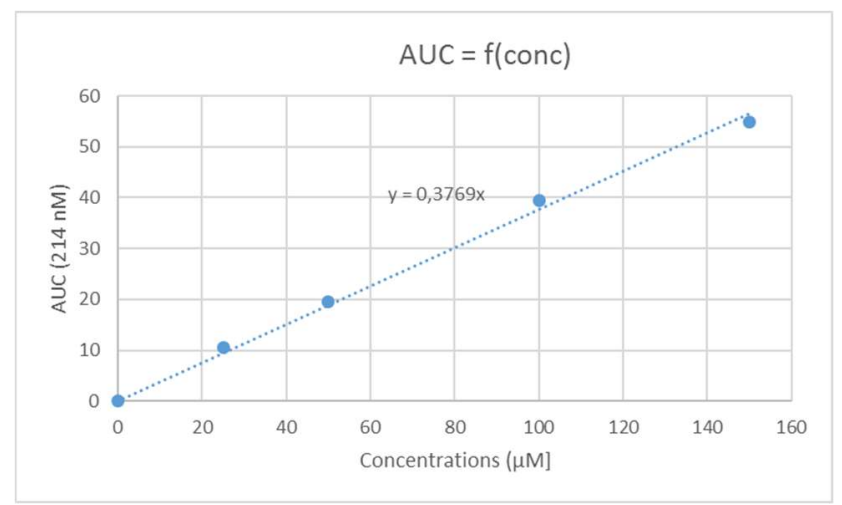

6.2. Preparation of solutions of ligand $\mathbf{4 4}$ in water

Solution 1: $1 \mathrm{mg}$ of ligand 44 in $500 \mu \mathrm{L}$ of water

Solution 2: $1 \mathrm{mg}$ of ligand $44 \mathrm{in} 1 \mathrm{~mL}$ of water

Protocol:

- $1 \mathrm{mg}$ of ligand 44 is solubilized in $\mathrm{x} \mathrm{mL}$ of water $(\mathrm{x}=500 \mu \mathrm{L}$ or $1 \mathrm{~mL})$

- after $5 \mathrm{~min}$ of wortexing, the solution is put under sonication at $25^{\circ} \mathrm{C}$ during $15 \mathrm{~min}$ (this step is done twice)

- the solution is centrifuged at $3000 \mathrm{rpm}$ during $10 \mathrm{~min}$

- $20 \mu \mathrm{L}$ of the supernatant are collected and injected in an analytical HPLC apparatus (reverse phase)

6.3. Results

\begin{tabular}{|c|c|c|}
\hline Solutions & AUC $(214 \mathrm{~nm})$ & Corresponding conc. $(\mu \mathrm{M})$ \\
\hline 1 & 2.06671 & 5.5 \\
\hline 2 & 1.85453 & 4.9 \\
\hline
\end{tabular}

Water solubility of ligand $44: 3.8 \mathrm{mg} / \mathrm{L}$ 